\title{
Commuting tuples in reductive groups and their maximal compact subgroups
}

\author{
Alexandra Pettet \\ JUAN SOUTO
}

Let $G$ be a reductive algebraic group and $K \subset G$ a maximal compact subgroup. We consider the representation spaces $\operatorname{Hom}\left(\mathbb{Z}^{k}, K\right)$ and $\operatorname{Hom}\left(\mathbb{Z}^{k}, G\right)$ with the topology induced from an embedding into $K^{k}$ and $G^{k}$, respectively. The goal of this paper is to prove that $\operatorname{Hom}\left(\mathbb{Z}^{k}, K\right)$ is a strong deformation retract of $\operatorname{Hom}\left(\mathbb{Z}^{k}, G\right)$.

20G20; 55P99

\section{Introduction $^{\dagger}$}

By a commuting $k$-tuple in a group $G$ we mean a $k$-tuple $\left(a_{1}, \ldots, a_{k}\right) \in G^{k}$ with $a_{i} a_{j}=a_{j} a_{i}$ for all $i$ and $j$. Observe that any such commuting $k$-tuple determines uniquely a homomorphism

$$
\mathbb{Z}^{k} \rightarrow G, \quad\left(n_{1}, \ldots, n_{k}\right) \mapsto a_{1}^{n_{1}} \cdots a_{k}^{n_{k}} .
$$

In fact, we obtain in this way a bijection between $\operatorname{Hom}\left(\mathbb{Z}^{k}, G\right)$ and the set of commuting $k$-tuples in $G$.

Suppose now that $G$ is a Lie group and that $K \subset G$ is a closed subgroup. Endowing $\operatorname{Hom}\left(\mathbb{Z}^{k}, G\right) \subset G^{k}$ and $\operatorname{Hom}\left(\mathbb{Z}^{k}, K\right) \subset K^{k}$ with the subspace topology, we consider the embedding

$$
\operatorname{Hom}\left(\mathbb{Z}^{k}, K\right) \hookrightarrow \operatorname{Hom}\left(\mathbb{Z}^{k}, G\right)
$$

induced by the inclusion of $K$ into $G$. The goal of this paper is to prove that the map (1.1) is a homotopy equivalence if $G$ is a reductive algebraic group and $K \subset G$ is a maximal compact subgroup.

Before stating our main result precisely, recall that a weak retraction of a topological space $X$ to a subspace $A$ is a continuous map

$$
F:[0,1] \times X \rightarrow X, \quad(t, x) \mapsto F_{t}(x)
$$

†Per me si va ne la cittá dolente, per me si va ne l'etterno dolore, per me si va tra la perduta gente. 
with $F_{0}=\operatorname{Id}_{X}$ and $F_{1}(X) \subset A$, and such that $F_{t}(A) \subset A$ for all $t$.

Theorem 1.1 Let $G$ be the group of complex or real points of a reductive algebraic group, defined over $\mathbb{R}$ in the latter case, and $K \subset G$ a maximal compact subgroup. For all $k$ there is a weak retraction of $\operatorname{Hom}\left(\mathbb{Z}^{k}, G\right)$ to $\operatorname{Hom}\left(\mathbb{Z}^{k}, K\right)$.

Before going further, recall that the presence of a weak retraction implies that the inclusion of $\operatorname{Hom}\left(\mathbb{Z}^{k}, K\right)$ into $\operatorname{Hom}\left(\mathbb{Z}^{k}, G\right)$ is a homotopy equivalence (Hatcher [18, page 18]). Notice also that $\operatorname{Hom}\left(\mathbb{Z}^{k}, K\right)$ and $\operatorname{Hom}\left(\mathbb{Z}^{k}, G\right)$ are algebraic varieties and hence homeomorphic to CW-complexes (Whitney [38]). It follows thus from Whitehead's Theorem [18, page 346] that in fact $\operatorname{Hom}\left(\mathbb{Z}^{k}, K\right)$ is a strong deformation retract of $\operatorname{Hom}\left(\mathbb{Z}^{k}, G\right)$.

Corollary 1.2 If $G$ is the group of complex or real points of a reductive algebraic group, defined over $\mathbb{R}$ in the latter case, and $K \subset G$ a maximal compact subgroup, then $\operatorname{Hom}\left(\mathbb{Z}^{k}, K\right)$ is a strong deformation retract of $\operatorname{Hom}\left(\mathbb{Z}^{k}, G\right)$ for all $k$.

If we replace the free abelian group $\mathbb{Z}^{k}$ by the free group $\mathbb{F}_{k}$, then the analogues of Theorem 1.1 and Corollary 1.2 follow directly from the well-known fact that $G$ retracts to $K$. It is worth noticing that such an argument cannot be applied to prove Theorem 1.1 because in general there is no retraction of $G$ to $K$ that preserves commutativity (Souto [34]).

It goes without saying that for a general finitely generated group $\Gamma$, the inclusion of $\operatorname{Hom}(\Gamma, K)$ into $\operatorname{Hom}(\Gamma, G)$ is not a homotopy equivalence. For example, if $\Gamma \subset \mathrm{SL}_{2} \mathbb{C}$ is a cocompact lattice then it follows from Mostow's Rigidity Theorem - or already from Weil's Local Rigidity Theorem - that the component of $\operatorname{Hom}\left(\Gamma, \mathrm{SL}_{2} \mathbb{C}\right)$ containing the tautological representation $\Gamma \hookrightarrow \mathrm{SL}_{2} \mathbb{C}$ consists of conjugates of this representation and hence is disjoint from $\operatorname{Hom}\left(\Gamma, \mathrm{SU}_{2}\right)$. Also, invariance of the Euler number implies that there are components of $\operatorname{Hom}\left(\pi_{1}(\Sigma), \mathrm{PSL}_{2} \mathbb{R}\right)$ that do not intersect $\operatorname{Hom}\left(\pi_{1}(\Sigma), \mathrm{SO}_{2}\right)$, where $\Sigma$ is a closed orientable surface of genus at least 2 (Goldman [14]).

Remark The proof of Theorem 1.1 applies, with only minor modifications, if we replace $\mathbb{Z}^{k}$ by any other finitely generated abelian group. In a subsequent paper, Lior Silberman and the second author use the results in the paper to show that Theorem 1.1 still holds true if $\mathbb{Z}^{k}$ is replaced by for instance a free nilpotent group or a Heisenberg group. 
Consequences of Theorem 1.1 Recall that the space $\operatorname{Hom}\left(\mathbb{Z}^{k}, G\right)$ may be identified with the space of (framed) flat $G$-connections on the $k$-torus modulo based gauge transformations. As such, these and other related spaces play a role in, for example, gauge theory, mathematical physics and symplectic geometry; see Jeffrey's nice survey describing these different points of view [21]. In fact, it was the work of Witten [40; 41] which motivated Borel, Friedman and Morgan [9], and Kac and Smilga [22] to initiate the systematic study of the topological properties of the spaces $\operatorname{Hom}\left(\mathbb{Z}^{k}, G\right)$. While the papers [9] and [22] concentrate on understanding the number of connected components of $\operatorname{Hom}\left(\mathbb{Z}^{k}, G\right)$ for certain values of $k$, higher topological invariants of these spaces, such as cohomology groups, were first investigated by Adem and Cohen in [1]. Starting with the latter paper, there have been a wealth of results in this direction: Adem, Cohen and Gómez [2; 3], Adem, Cohen and Torres-Giese [4], Baird [5], Baird, Jeffrey and Selik [6], Crabb [12], Higuera [19], Gómez, Pettet and Souto [15] and Torres-Giese and Sjerve [37].

At this point it should be mentioned that all results proved in the papers just mentioned concern, almost without exception, the space of commuting tuples in compact groups. Combining these results with Theorem 1.1 we obtain hence the corresponding results for reductive groups. We give just a few examples of this strategy:

Corollary 1.3 Suppose that $G$ is the set of complex points of a semisimple algebraic group. The space $\operatorname{Hom}\left(\mathbb{Z}^{k}, G\right)$ is connected if and only if one of the following conditions is satisfied:

(1) $k=1$ and $G$ is connected,

(2) $k=2$ and $G$ is connected and simply connected, or

(3) $k \geq 3$ and $G$ is a product of copies of $\mathrm{SL}_{n} \mathbb{C}$ and $\mathrm{Sp}_{n} \mathbb{C}$.

Proof Let $K$ be a maximal compact subgroup of $G$, and recall that $K$ is homotopy equivalent to $G$. It is due to $\operatorname{Kac}$ and $\operatorname{Smilga}[22]$ that $\operatorname{Hom}\left(\mathbb{Z}^{k}, K\right)$ is connected if and only if $k=1$ and $K$ is connected, $k=2$ and $K$ is connected and simply connected, or $k \geq 3$ and $K$ is a product of copies of $\mathrm{SU}_{n}$ and $\mathrm{Sp}_{n}$ for some $n \geq 1$. As $K$ determines $G$ up to isomorphism (see Serre [31, VIII]), and as $\mathrm{SU}_{n}$ and $\mathrm{Sp}_{n}$ are maximal compact subgroups of $\mathrm{SL}_{n} \mathbb{C}$ and $\mathrm{Sp}_{n} \mathbb{C}$, the claim follows from Theorem 1.1.

Remark In the proof of Corollary 1.3 we have used that complex reductive groups are determined by their maximal compact subgroups; this is not true in the real case: $\mathrm{O}_{n}(\mathbb{R})$ is a maximal compact subgroup of both $\mathrm{GL}_{n} \mathbb{R}$ and $\mathrm{SO}_{(n, 1)} \mathbb{R}$. Theorem 1.1 implies that the varieties of commuting $k$-tuples of these two groups are homotopy equivalent; at first glance, the authors find this somehow surprising. 
If $K$ is a compact group, then the connected component $\operatorname{Hom}\left(\mathbb{Z}^{k}, K\right)_{\mathbb{1}}$ of the trivial representation in $\operatorname{Hom}\left(\mathbb{Z}^{k}, K\right)$ is much better understood than is the whole space. For instance, its fundamental group [15] and rational cohomology groups [5] are known.

Corollary 1.4 If $G$ is a reductive group, complex or real, then $\pi_{1}\left(\operatorname{Hom}\left(\mathbb{Z}^{k}, G\right)_{\mathbb{1}}\right)=$ $\pi_{1}(G)^{k}$ for all $k$. Here, $\operatorname{Hom}\left(\mathbb{Z}^{k}, G\right)_{\mathbb{1}}$ is the connected component in $\operatorname{Hom}\left(\mathbb{Z}^{k}, G\right)$ of the trivial representation.

Proof Let $K$ be a maximal compact subgroup of $G$. It follows from Theorem 1.1 that $\operatorname{Hom}\left(\mathbb{Z}^{k}, G\right)_{\mathbb{1}}$ and $\operatorname{Hom}\left(\mathbb{Z}^{k}, K\right)_{\mathbb{1}}$ are homotopy equivalent and hence have isomorphic fundamental groups. By [15] we have that $\pi_{1}\left(\operatorname{Hom}\left(\mathbb{Z}^{k}, K\right)_{\mathbb{1}}\right)$ and $\pi_{1}(K)^{k}$ are isomorphic. Since $K$ and $G$ are also homotopy equivalent, the result follows.

Corollary 1.5 Let $G$ be a connected reductive group, complex or real, $T \subset G$ a maximal torus, and $W=\mathcal{N}_{G}(T) / \mathcal{Z}_{G}(T)$ the Weyl-group of $G$. We have

$$
H^{*}\left(\operatorname{Hom}\left(\mathbb{Z}^{k}, G\right)_{\mathbb{1}}, F\right) \simeq H^{*}\left(G / T \times T^{k}, F\right)^{W},
$$

where $F$ is any field with characteristic either 0 or relatively prime to the order $W$.

Proof Let $K$ be a maximal compact subgroup of $G$ such that $T_{K}=T \cap K$ is a maximal torus in $K$; notice that this implies that the inclusion $T_{K} \rightarrow T$ is a homotopy equivalence. In particular, the inclusion

$$
K / T_{K} \times T_{K}^{k} \rightarrow G / T \times T^{k}
$$

is a $W$-equivariant homotopy equivalence and hence we have:

$$
H^{*}\left(K / T_{K} \times T_{K}^{k}, F\right)^{W} \simeq H^{*}\left(G / T \times T^{k}, F\right)^{W} .
$$

It is due to Baird [5, Theorem 4.3] that the left side of (1.2) is isomorphic to $H^{*}\left(\operatorname{Hom}\left(\mathbb{Z}^{k}, K\right)_{\mathbb{1}}, F\right)$ and hence to $H^{*}\left(\operatorname{Hom}\left(\mathbb{Z}^{k}, G\right)_{\mathbb{1}}, F\right)$ by Theorem 1.1.

Remark From the proof of Theorem 1.1 we obtain the following description of $\operatorname{Hom}\left(\mathbb{Z}^{k}, G\right)_{\mathbb{1}}$ : a commuting tuple $\left(a_{1}, \ldots, a_{k}\right)$ belongs to $\operatorname{Hom}\left(\mathbb{Z}^{k}, G\right)_{\mathbb{1}}$ if and only if there is a torus $T \subset G$ containing the semisimple part of the Jordan decomposition of $a_{i}$ for all $i$.

Topological considerations aside, observe that, with notation as before, we can also consider $\operatorname{Hom}\left(\mathbb{Z}^{k}, G\right)$ as an algebraic variety. In [29], Richardson proved that $\operatorname{Hom}\left(\mathbb{Z}^{2}, G\right)$ is an irreducible variety whenever $G$ is the group of complex points of a connected, simply connected and semisimple algebraic group. Combining Richardson's result with the fact that an irreducible complex variety is connected in the Hausdorff topology, we deduce from Corollary 1.3: 
Corollary 1.6 Let $G$ be the group of complex points of a semisimple algebraic group. The variety of commuting pairs in $G$ is irreducible if and only if $G$ is connected and simply connected.

Similarly, we obtain from Corollary 1.3 that for $G$ simple and $k \geq 3$, the variety $\operatorname{Hom}\left(\mathbb{Z}^{k}, G\right)$ is reducible, unless possibly for $G=\mathrm{SL}_{n} \mathbb{C}$ and $G=\mathrm{Sp}_{n} \mathbb{C}$. This result is far from optimal: it follows easily from the work of Gerstenhaber [13] that $\operatorname{Hom}\left(\mathbb{Z}^{k}, \mathrm{SL}_{n} \mathbb{C}\right)$ is reducible for $k, n \geq 4$; see also Guralnick [17] and the references therein for more recent results.

Remark For comparison, it is easy to see that $\operatorname{Hom}\left(\mathbb{Z}^{k}, \mathrm{SU}_{n}\right)$ is an irreducible analytic space for all $k$ and $n$, meaning that it is not the union of two proper analytic subsets. To see that this is the case, let $\mathbb{T}$ be the group of diagonal matrices in $\mathrm{SU}_{n}$ and observe that the analytic map

$$
\mathrm{SU}_{n} \times \mathbb{T}^{k} \rightarrow \operatorname{Hom}\left(\mathbb{Z}^{k}, \mathrm{SU}_{n}\right), \quad\left(g, a_{1}, \ldots, a_{k}\right) \mapsto\left(g a_{1} g^{-1}, \ldots, g a_{k} g^{-1}\right)
$$

is surjective. The domain is a connected analytic manifold and hence irreducible as an analytic space; the image of an irreducible analytic space under an analytic map is irreducible.

Outline of the paper We will divide the proof of Theorem 1.1, and with it the whole of this paper, into two distinct parts, as the arguments lend themselves naturally to this partition. In the second part of the paper (Sections 7-8), we prove that there is a commutativity-preserving retraction of $G$ to $G^{s}$, the subset consisting of semisimple elements. It follows directly that there is a retraction of $\operatorname{Hom}\left(\mathbb{Z}^{k}, G\right)$ to $\operatorname{Hom}\left(\mathbb{Z}^{k}, G^{s}\right)$, the space of commuting semisimple $k$-tuples. In the first part of the paper (Sections 2-6), we prove that $\operatorname{Hom}\left(\mathbb{Z}^{k}, K\right)$ is a retract of $\operatorname{Hom}\left(\mathbb{Z}^{k}, G^{s}\right)$, completing the proof of Theorem 1.1.

Sections 2-6 The goal of these sections is to prove that the space $\operatorname{Hom}\left(\mathbb{Z}^{k}, G^{s}\right)$ of commuting semisimple tuples retracts to $\operatorname{Hom}\left(\mathbb{Z}^{k}, K\right)$.

We stratify $\operatorname{Hom}\left(\mathbb{Z}^{k}, G^{s}\right)$ according to the conjugacy class of the stabilizer under the action by conjugation $G \curvearrowright \operatorname{Hom}\left(\mathbb{Z}^{k}, G\right)$ and note that this stratification has finitely many strata; this is the content of Section 2.

To any stratum $Z$ of $\operatorname{Hom}\left(\mathbb{Z}^{k}, G^{s}\right)$ we associate an abelian subgroup $A_{Z} \subset G$ and a surjective map

$$
\left(G \times A_{Z}^{k}\right) / \mathcal{N}_{G}\left(A_{Z}\right) \rightarrow Z,
$$


where $\mathcal{N}_{G}\left(A_{Z}\right)$ is the normalizer of $A_{Z}$ in $G$. The groups $A_{Z}$ are described in Section 3, where we discuss the well-centered groups: these are the diagonalizable subgroups of $G$ that are equal to the center of their centralizer. The main result of this section is that the sets $\mathcal{G}$ and $\mathcal{K}$ of well-centered groups in $G$ and $K$ are manifolds with finitely many connected components, and that there is an injective homotopy equivalence $\mathcal{K} \hookrightarrow \mathcal{G}$. The map (1.3) and some of its properties are discussed in Section 4. The central observation there is that the domain of (1.3) is a flat bundle over a connected component of the manifold $\mathcal{G}$ of well-centered groups. The flatness of this bundle allows horizontal lifting of any retraction of $\mathcal{G}$ to $\mathcal{K}$. We prove that one can choose the retractions of $\mathcal{G}$ to $\mathcal{K}$ in such a way that the associated horizontal lifts descend under (1.3) to a retraction of $\operatorname{Hom}\left(\mathbb{Z}^{k}, G^{s}\right)$ to $\operatorname{Hom}\left(\mathbb{Z}^{k}, K\right)$. These steps make up Sections 5 and 6; some of the painful details of Section 5 are suppressed until the Appendix.

Sections 7-8 These two sections are devoted to proving that there is a commutativitypreserving retraction of $G$ into the set $G^{s}$ of semisimple elements. The basic idea is to associate to every element of $G$ its multiplicative Jordan decomposition and to homotope away the unipotent part using the logarithm. In doing so, we face the problem that the Jordan decomposition is not continuous. We bypass this difficulty by constructing a continuous approximation of the Jordan decomposition. More concretely, letting $G$ act on itself and on $G \times G$ by conjugation, we prove:

Theorem 1.7 Let $G \subset \mathrm{SL}_{n} \mathbb{C}$ be an algebraic group and $H_{1}, \ldots, H_{r} \subset G$ a finite collection of algebraic subgroups of $G$. For every $\epsilon>0$ there is a continuous $G-$ equivariant map

$$
G \rightarrow G \times G, \quad g \mapsto\left(g_{s}^{\epsilon}, g_{u}^{\epsilon}\right)
$$

satisfying the following properties for all $g \in G$ :

(1) $g=g_{s}^{\epsilon} g_{u}^{\epsilon}=g_{u}^{\epsilon} g_{s}^{\epsilon}$.

(2) $g_{s}^{\epsilon}$ and $g_{u}^{\epsilon}$ are polynomials in $g$, ie, they belong to $\mathbb{C}[g] \subset M_{n} \mathbb{C}$.

(3) If $h \in G$ commutes with $g \in G$, then $h$ also commutes with $g_{s}^{\epsilon}$ and $g_{u}^{\epsilon}$.

(4) $g_{s}^{\epsilon}$ is semisimple and $g_{u}^{\epsilon}-$ Id has spectral radius at most $\epsilon$.

(5) If $K \subset G$ is a maximal compact group then $g_{s}^{\epsilon}, g_{u}^{\epsilon} \in K$ for all $g \in K$.

(6) $g_{s}^{\epsilon}, g_{s}^{u} \in H_{i}$ for all $g \in H_{i}$.

(7) If moreover $G$ is defined over $\mathbb{R}$, then $\bar{g}_{s}^{\epsilon}=\overline{g_{s}^{\epsilon}}$ and $\bar{g}_{u}^{\epsilon}=\overline{g_{u}^{\epsilon}}$, where - is complex conjugation. 
Remark Notice that equivariance in Theorem 1.7 means that

$$
\left(h g h^{-1}\right)_{s}^{\epsilon}=h g_{s}^{\epsilon} h^{-1} \quad \text { and } \quad\left(h g h^{-1}\right)_{u}^{\epsilon}=h g_{u}^{\epsilon} h^{-1}
$$

for all $g, h \in G$. Observe also that the closure of $K^{G}=\bigcup_{g \in G} g K^{-1}$ in $G$ contains non-trivial unipotent elements. In particular, Theorem 1.7(5) cannot be strengthened to get $g_{s}^{\epsilon}=g_{s}$ for all $g \in K$. A similar argument shows that (6) cannot be strengthened to allow infinite collections of Zariski closed subgroups.

We refer to the decomposition $g=g_{s}^{\epsilon} g_{u}^{\epsilon}$ provided by Theorem 1.7 as the approximated Jordan decomposition of $g \in G$. It shares the commutativity properties of the standard Jordan decomposition $g=g_{s} g_{u}$, and likewise it behaves well with respect to conjugation, and has the property that both components are polynomials in $g$. The distinct advantage of the approximated version is that it is continuous. The main compromise is that we can only say that the linear transformation $g_{u}^{\epsilon}-$ Id has spectral radius bounded above by $\epsilon$; in the standard decomposition, the spectral radius of $g_{u}$ is vanishing, ie, $g_{u}$ is unipotent. Recall that the spectral radius of a matrix is the maximum of the norms of its eigenvalues.

Concluding remark The strategy of the proof of Theorem 1.1 can likely be adapted to shed some light on whether or not $\operatorname{Hom}(\Gamma, G)$ retracts to $\operatorname{Hom}(\Gamma, K)$, when $\Gamma$ is a right angled Artin group (Charney [11]). The latter problem is particularly interesting as right angled Artin groups in some sense interpolate between free groups and free abelian groups; and although for these latter two classes of groups, $\operatorname{Hom}(\Gamma, G)$ retracts to $\operatorname{Hom}(\Gamma, K)$, each does so for its own distinct reason. Finally, much of the content of Sections 2-6 seems well posed for the study of other problems, such as computing cohomological invariants of $\operatorname{Hom}\left(\mathbb{Z}^{k}, G\right)$.

Real vs complex Most of the work in this paper is devoted to prove Theorem 1.1 for complex groups. The proof for real groups is almost identical; it suffices to add only a few remarks here and there. When needed, we will discuss the real case of the results in a section only after the complex case has been completely treated.

\section{Acknowledgements}

The authors thank the universities of Chicago, Stanford, Michigan and British Columbia for allowing them to teach linear algebra. We thank Alejandro Ádem, Mladen Bestvina, Bill Fulton, José Manuel Gómez, Vincent Guirardel, Mel Hochster, Mircea Mustata, Lior Silberman, Ralf Spatzier and Enrique Torres-Giese for very helpful and interesting conversations. This paper would not have been possible without Michel Brion's, Gopal Prasad's and Zinovy Reichstein's willingness to answer our many questions. 
The first author has been partially supported by NSF grant DMS-0856143 and NSF RTG grant DMS-0602191. The second author has been partially supported by NSF grant DMS-0706878, NSF CAREER award 0952106 and by the Alfred P Sloan Foundation.

\section{Action by conjugation}

By an algebraic group $G$ we mean the set of complex points of a linear algebraic group; we say that $G$ is connected if it is connected in the Zariski topology. If $G$ is defined over $\mathbb{R}$, then we denote by $G(\mathbb{R})$ the set of real points. Note that $G$ and $G(\mathbb{R})$ are both Lie groups satisfying

$$
\operatorname{dim}_{\mathbb{C}}(G)=\operatorname{dim}_{\mathbb{R}}(G(\mathbb{R})) .
$$

As Lie groups, both $G$ and $G(\mathbb{R})$ have finitely many connected components. In fact, $G$ is connected as a Lie group if and only if it is connected in the sense above. Notice however that the set $G(\mathbb{R})$ of real points of a connected algebraic group defined over $\mathbb{R}$ does not need to be a connected Lie group.

We will denote by $K$ a maximal compact subgroup of $G$ and by $K(\mathbb{R})$ a maximal compact subgroup of $G(\mathbb{R})$. It is well-known that maximal compact subgroups exist and that any two are conjugate to each other; in fact, any two maximal compact subgroups are conjugate by elements in the identity component $G_{0}$ (resp. $\left.G(\mathbb{R})_{0}\right)$ of the ambient group $G$ (resp. $G(\mathbb{R})_{0}$ ). It is also well known that the inclusion $K \hookrightarrow G$ (resp. $K(\mathbb{R}) \hookrightarrow G(\mathbb{R})$ ) of a maximal compact subgroup into the ambient group is a homotopy equivalence.

Recall that the unipotent radical $\mathcal{R}_{u}(G)$ of an algebraic group $G$ is the largest normal connected unipotent subgroup thereof. If $\mathcal{R}_{u}(G)$ is trivial, then $G$ is said to be reductive. The identity component $G_{0}$ of $G$ is itself an algebraic group. A real reductive group is the group of real points of a reductive algebraic group defined over $\mathbb{R}$.

Algebraic varieties will be, unless explicitly stated, complex affine varieties; in fact, varieties will be more often than not actually embedded inside affine space. In particular, variety is often just synonymous to Zariski closed subset of $\mathbb{C}^{n}$ for some $n$.

Remark Throughout most of this paper we will be interested in topological properties of groups and spaces with respect to the analytic topology. Whenever we work with the Zariski topology we will make this explicit by writing Zariski closed, Zariski open, etc.

We refer to Borel [7] and Shafarevich [33] for basic facts about algebraic groups and varieties. See Borel [8] for the relation between complex and real algebraic groups. 


\subsection{The variety of commuting tuples}

Given a linear algebraic group $G$, we identify the $\operatorname{set} \operatorname{Hom}\left(\mathbb{Z}^{k}, G\right)$ of all homomorphisms $\mathbb{Z}^{k} \rightarrow G$ with the variety of commuting $k$-tuples in $G$ :

$$
\operatorname{Hom}\left(\mathbb{Z}^{k}, G\right)=\left\{\left(a_{1}, \ldots, a_{k}\right) \in G^{k} \mid a_{i} a_{j}=a_{j} a_{i} \forall i, j\right\} .
$$

In this way, $\operatorname{Hom}\left(\mathbb{Z}^{k}, G\right)$ becomes an affine complex algebraic variety. The identification between $\operatorname{Hom}\left(\mathbb{Z}^{k}, G\right)$ and the variety of commuting $k$-tuples in $G$ depends on the choice of basis of $\mathbb{Z}^{k}$. It is well-known, and otherwise easy to see, that the structure of $\operatorname{Hom}\left(\mathbb{Z}^{k}, G\right)$ as an algebraic variety does not depend on the chosen embedding.

As an analytic space, the variety $\operatorname{Hom}\left(\mathbb{Z}^{k}, G\right)$ also has the associated Hausdorff topology. Notice that this topology agrees with the topology induced by embedding $\operatorname{Hom}\left(\mathbb{Z}^{k}, G\right)$ into $G^{k}$, when considering the algebraic group $G$ as a complex Lie group.

Remark We will denote elements in $\operatorname{Hom}\left(\mathbb{Z}^{k}, G\right)$ by $\rho$ if we consider them as homomorphisms and by $\left(a_{1}, \ldots, a_{k}\right)$ if we consider them as tuples. With the hope that no confusion arises, we feel free to pass from one form of notation to the other.

The group $G$ acts on $\operatorname{Hom}\left(\mathbb{Z}^{k}, G\right)$ by conjugation:

$$
\begin{aligned}
G \times \operatorname{Hom}\left(\mathbb{Z}^{k}, G\right) & \rightarrow \operatorname{Hom}\left(\mathbb{Z}^{k}, G\right), \\
\left(g,\left(a_{1}, \ldots, a_{k}\right)\right) & \mapsto\left(g a_{1} g^{-1}, \ldots, g a_{k} g^{-1}\right) .
\end{aligned}
$$

In this section we study a few properties of this action. However, we first remind the reader of a few facts on algebraic varieties and algebraic actions of algebraic groups.

\subsection{Quotients}

Recall that it is a theorem of Cartan that any closed subgroup $H$ of a Lie group $G$ is also a Lie group. It is well known that the coset space $G / H$ is a manifold that admits a unique smooth structure with respect to which the quotient map

$$
\pi: G \rightarrow G / H
$$

is the projection associated to a principal bundle with structure group $H$.

Similarly, any Zariski closed subgroup $H$ of a (complex) algebraic group $G$ is also an algebraic group. In this setting, the coset space $G / H$ has a unique structure as a quasiprojective variety such that:

- the map $\pi: G \rightarrow G / H$ is a morphism, 
- the Zariski topology of $G / H$ is the quotient topology, and

- for any Zariski open set $U \subset G / H$, the pull-back map is an isomorphism between the algebras of functions on $U$ and of $H$-invariant functions on $\pi^{-1}(U)$.

While it is important to keep in mind that $G / H$ is in general not affine, but rather only quasiprojective, the following result due to Richardson [28] will have the consequence that in all cases of interest in this paper, the coset space is in fact affine:

Theorem 2.1 (Richardson [28]) Suppose $G$ is a reductive algebraic group and $H \subset$ $G$ is a Zariski closed subgroup. Then the following are equivalent:

- The quasi-projective variety $G / H$ is isomorphic to an affine variety.

- $H$ is reductive.

Still assuming that $H$ is a Zariski closed subgroup of $G$, it is worth mentioning that the subjacent analytic space of the variety $G / H$ is a smooth manifold which, as one would expect, agrees with the manifold structure mentioned above.

We refer the reader to [16] and [7;26] for basic properties of quotient spaces in the smooth and algebraic categories.

\subsection{Actions of reductive groups}

Suppose that $G$ is an algebraic group and $X$ an affine variety, both complex. An algebraic action $G \curvearrowright X$ of $G$ on $X$ is a morphism of varieties

$$
G \times X \rightarrow X
$$

satisfying the usual properties of an action of a group on a set. We refer to [10] for a gentle introduction to the basic properties of algebraic actions.

Given an (algebraic) action $G \curvearrowright X$ and a point $x \in X$ we denote by $\operatorname{Orbit}_{G}(x)$ and $\operatorname{Stab}_{G}(x)$ the orbit and the stabilizer of $x$, respectively. We summarize a few properties of the orbits and stabilizers of algebraic groups:

(1) $\operatorname{Stab}_{G}(x)$ is Zariski closed in $G$; hence $\operatorname{Stab}_{G}(x)$ is an algebraic subgroup of $G$.

(2) $\operatorname{Orbit}_{G}(x)$ is a smooth quasi-projective variety.

(3) The map $G / \operatorname{Stab}_{G}(x) \rightarrow \operatorname{Orbit}_{G}(x)$ given by $g \operatorname{Stab}_{G}(x) \mapsto g x$ is an isomorphism of quasi-projective varieties. 
Notice that if $\operatorname{Orbit}_{G}(x)$ happens to be a Zariski closed subset of the affine variety $X$, then $\operatorname{Orbit}_{G}(x)$ is itself an affine variety. We deduce hence from (3) and Theorem 2.1:

Lemma 2.2 Suppose that $G \curvearrowright X$ is an algebraic action of a reductive group $G$ on an affine variety $X$. If the orbit $\operatorname{Orbit}_{G}(x)$ of some $x \in X$ is Zariski closed, then $\operatorname{Stab}_{G}(x)$ is reductive.

It should be remarked that there are actions of reductive groups with reductive stabilizers for which the orbits are not closed; this is so because the image of an affine variety under a morphism is in general not Zariski closed.

\subsection{Orbit types and principal orbits}

Suppose that $G \curvearrowright X$ is an action of a group on a set. We say that $x, y \in X$ have the same orbit type if the stabilizers $\operatorname{Stab}_{G}(x)$ and $\operatorname{Stab}_{G}(y)$ are conjugate in $G$.

Remark Suppose for the sake of concreteness that $G$ is an algebraic group, $X$ is an algebraic variety, and $G \curvearrowright X$ is an algebraic action. Notice that $x, y \in X$ have the same orbit type if and only if their orbits $\operatorname{Orbit}_{G}(x)$ and $\operatorname{Orbit}_{G}(y)$ are $G$-equivariantly isomorphic.

A topological (resp. algebraic) action $G \curvearrowright X$ of a Lie group (resp. algebraic group) $G$ on a topological space (resp. algebraic variety) $X$ is said to have a principal orbit if there is a dense open (resp. Zariski dense Zariski open) set $U \subset X$ such that any two $x, y \in U$ have the same orbit type.

It is well-known that actions of compact Lie groups on compact manifolds have principal orbits. As a general rule, reductive groups are the algebraic analogue of compact Lie groups. In this spirit, the following result due to Richardson [27] asserts that under suitable conditions, algebraic actions of reductive groups on affine varieties have principal orbits as well:

Theorem 2.3 (Richardson [27]) Let $G \curvearrowright X$ be an algebraic action of a reductive algebraic group $G$ on an irreducible affine algebraic variety $X$. If there is a smooth point $x \in X$ with $\operatorname{Stab}_{G}(x)$ reductive, then the action $G \curvearrowright X$ has a principal orbit.

In the setting of actions of compact Lie groups on compact manifold $M$, Mostow [24] proved that the stabilizers of points near $x \in M$ are conjugate to subgroups of the stabilizer of $x$. In [27], Richardson also obtained an analogue of this assertion for algebraic actions of reductive groups: 
Theorem 2.4 (Richardson [27]) Let $G \curvearrowright X$ be an algebraic action of a reductive algebraic group $G$ on an affine algebraic variety $X$. Suppose that $x \in X$ is such that its orbit $\operatorname{Orbit}_{G}(x)$ is closed. Then there is a Zariski open set $U \subset X$ such that the stabilizer $\operatorname{Stab}_{G}(y)$ of every $y \in U$ is conjugate to a subgroup of $\operatorname{Stab}_{G}(x)$.

Theorem 2.4 is also a consequence of Luna's Slice Theorem [23], which asserts that

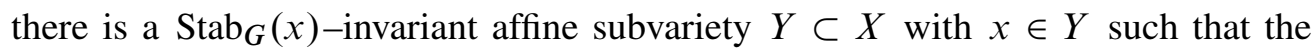
morphism

$$
(Y \times G) / \operatorname{Stab}_{G}(x) \rightarrow X
$$

is étale. In complex analytic terms, "étale" is synonymous with "local analytic isomorphism" (Mumford [25, III.5 Corollary 2]). In particular, Theorem 2.4 can be strengthened as follows: if $x_{n} \rightarrow x$ converges in the analytic topology, then there are $g_{n} \rightarrow \operatorname{Id}$ with $g_{n} \operatorname{Stab}_{G}\left(x_{n}\right) g_{n}^{-1} \subset \operatorname{Stab}_{G}(x)$.

\subsection{Centralizers of semisimple tuples}

Assume that $G$ is a reductive algebraic group and recall that we identify elements $\rho$ in $\operatorname{Hom}\left(\mathbb{Z}^{k}, G\right)$ with commuting $k$-tuples $\left(a_{1}, \ldots, a_{k}\right)$ in $G$. As we mentioned earlier, the group $G$ acts on $\operatorname{Hom}\left(\mathbb{Z}^{k}, G\right)$ by conjugation. The stabilizer of a representation $\rho$ under this action of $G$ is the centralizer of its image:

$$
\operatorname{Stab}_{G}(\rho)=\mathcal{Z}_{G}\left(\rho\left(\mathbb{Z}^{k}\right)\right),
$$

where, for $X \subset G$ :

$$
\mathcal{Z}_{G}(X)=\{g \in G \mid g x=x g \forall x \in X\} .
$$

A tuple $\left(a_{1}, \ldots, a_{k}\right) \in \operatorname{Hom}\left(\mathbb{Z}^{k}, G\right)$ is semisimple if each $a_{i}$ is. Since every abelian subgroup of $G$ generated by semisimple elements consists solely of semisimple elements [7] we have:

Lemma 2.5 $\rho \in \operatorname{Hom}\left(\mathbb{Z}^{k}, G\right)$ is semisimple if and only if $\rho\left(\mathbb{Z}^{k}\right)$ is an abelian subgroup consisting of semisimple elements.

We prove that the action $G \curvearrowright \operatorname{Hom}\left(\mathbb{Z}^{k}, G\right)$ has only finitely many orbit types of semisimple tuples.

Proposition 2.6 Let $G$ be a reductive algebraic group with identity component $G_{0}$. The actions of $G$ and $G_{0}$ on $\operatorname{Hom}\left(\mathbb{Z}^{k}, G\right)$ by conjugation have only finitely many orbit types of semisimple representations. 
The key ingredient in the proof of Proposition 2.6 is the following result due to Richardson:

Theorem 2.7 (Richardson [30]) Let $G$ be a reductive algebraic group and consider the action $G \curvearrowright \operatorname{Hom}\left(\mathbb{Z}^{k}, G\right)$ by conjugation. If $\rho \in \operatorname{Hom}\left(\mathbb{Z}^{k}, G\right)$ is semisimple, then Orbit $_{G}(\rho)$ is Zariski closed in $\operatorname{Hom}\left(\mathbb{Z}^{k}, G\right)$; hence, $\operatorname{Stab}_{G}(\rho)$ is reductive.

Proof of Proposition 2.6 Since $G_{0}$ has finite index in $G$, it suffices to prove that the action of $G_{0}$ has finitely many orbit types. Let $\mathcal{V}$ be the set of all irreducible, $G_{0}$-invariant affine subvarieties $V \subset \operatorname{Hom}\left(\mathbb{Z}^{k}, G\right)$ containing as a smooth point a semisimple tuple. Arguing by induction on the dimension, we will show that each $V \in \mathcal{V}$ consists of finitely many orbit types. The base case of the induction, namely $\operatorname{dim} V=0$, is clear, for these are exactly the tuples of central elements in $G_{0}$.

So, suppose that we have proved the claim for all elements in $\mathcal{V}$ of at most dimension $d-1$ and let $V \in \mathcal{V}$ be of dimension $d$. By assumption, $V$ contains as a smooth point a semisimple tuple $\rho$. By Theorem 2.7, $\operatorname{Stab}_{G_{0}}(\rho)$ is reductive. Hence, we can apply Theorem 2.3 and deduce that $G_{0} \curvearrowright V$ has a principal orbit. In other words, there is $U \subset V$ Zariski open such that for any two $\rho_{1}, \rho_{2} \in U$ the stabilizers $\operatorname{Stab}_{G_{0}}\left(\rho_{1}\right)$ and $\operatorname{Stab}_{G_{0}}\left(\rho_{2}\right)$ are conjugate. Hence it suffices to show that the affine $G_{0}$-invariant variety $V \backslash U$ contains finitely many orbit types of semisimple tuples; notice that $V \backslash U$ has at most dimension $d-1$. Consider the Zariski closure $V^{\prime}$ of the set of semisimple tuples in $V \backslash U$ and let $V^{\prime}=V_{1}^{\prime} \cup \cdots \cup V_{r}^{\prime}$ be the decomposition into irreducible components. Clearly $V^{\prime}$ is $G_{0}$-invariant; by the connectedness of $G_{0}$, each $V_{i}^{\prime}$ is also $G_{0}$-invariant. Moreover, each $V_{i}^{\prime}$ has at most dimension $d-1$ and contains a semisimple tuple as a smooth point, as the set of singular points is a subvariety. From the induction assumption, we deduce that each $V_{i}^{\prime}$ contains only finitely many orbit types of semisimple tuples. Since every semisimple tuple in $V \backslash U$ is contained in $V^{\prime}$, this proves that $V$ also contains only finitely many orbit types of semisimple tuples.

At this point we know that every affine irreducible $G_{0}$-invariant subvariety $V \subset$ $\operatorname{Hom}\left(\mathbb{Z}^{k}, G\right)$ containing as a smooth point a semisimple tuple contains only finitely many orbit types of semisimple tuples. As in the induction step, the Zariski closure of the set of semisimple tuples in $\operatorname{Hom}\left(\mathbb{Z}^{k}, G\right)$ is a finite union of such subvarieties. In particular, $\operatorname{Hom}\left(\mathbb{Z}^{k}, G\right)$ contains only finitely many $G_{0}$-orbit types of semisimple tuples, as claimed.

\subsection{Triangulations}

In later sections we will need the existence of suitable triangulations of the subset of $\operatorname{Hom}\left(\mathbb{Z}^{k}, G\right)$ consisting of semisimple representations. Before stating a precise result, 
we introduce some notation for the sets of semisimple elements and of elements that can be conjugated into some maximal compact subgroup $K \subset G$ :

$$
G^{s}=\{g \in G \mid g \text { semisimple }\}, \quad K^{G}=\bigcup_{g \in G} g K g^{-1} .
$$

We will also use the following notation:

$$
\begin{aligned}
\operatorname{Hom}\left(\mathbb{Z}^{k}, G^{s}\right) & =\left\{\left(a_{1}, \ldots, a_{k}\right) \in \operatorname{Hom}\left(\mathbb{Z}^{k}, G\right) \mid a_{i} \in G^{s} \forall i\right\}, \\
\operatorname{Hom}\left(\mathbb{Z}^{k}, K^{G}\right) & =\left\{\left(a_{1}, \ldots, a_{k}\right) \in \operatorname{Hom}\left(\mathbb{Z}^{k}, G\right) \mid a_{i} \in K^{G} \forall i\right\} .
\end{aligned}
$$

Remark Since every element in $K$ is semisimple, we have $K^{G} \subset G^{s}$.

We can now state the needed existence of triangulations:

Proposition 2.8 Suppose that $G$ is a reductive algebraic group, and let $K \subset G$ be a maximal compact subgroup. There is a triangulation of $\operatorname{Hom}\left(\mathbb{Z}^{k}, G^{s}\right)$ such that

$$
\mathcal{X}_{H}=\left\{\rho \in \operatorname{Hom}\left(\mathbb{Z}^{k}, G^{s}\right) \mid \exists g \in G \text { with } g H^{-1} \subset \operatorname{Stab}_{G}(\rho)\right\}
$$

is a subcomplex for every subgroup $H \subset G$. Moreover, the subsets $\operatorname{Hom}\left(\mathbb{Z}^{k}, K\right)$ and $\operatorname{Hom}\left(\mathbb{Z}^{k}, K^{G}\right)$ are also subcomplexes.

Recall that a triangulation of a topological space is a homeomorphism to the geometric realization of a simplicial complex. Abusing notation, we will not distinguish between simplicial complexes, their geometric realizations, and triangulations.

Before launching into the proof of Proposition 2.8, we need a few preliminary observations.

Lemma 2.9 The set $\left\{\mathcal{X}_{H} \mid H \subset G\right\}$ of subsets of $\operatorname{Hom}\left(\mathbb{Z}^{k}, G^{s}\right)$ is finite.

Proof By Proposition 2.6, the action $G \curvearrowright \operatorname{Hom}\left(\mathbb{Z}^{k}, G^{s}\right)$ has only finitely many orbit types. In other words, there is a finite set $H_{1}, \ldots, H_{r}$ of subgroups of $G$ such that for all $\rho \in \operatorname{Hom}\left(\mathbb{Z}^{k}, G^{s}\right)$ there are $i \in\{1, \ldots, r\}$ and $g \in G$ with $g H_{i} g^{-1}=\operatorname{Stab}_{G}(\rho)$. It follows that $\mathcal{X}_{H}$ is precisely the union of the sets $\mathcal{X}_{H_{i}}$ for which there is $g \in G$ with $g \mathrm{Hg}^{-1} \subset H_{i}$. Clearly, only finitely many subsets of $\operatorname{Hom}\left(\mathbb{Z}^{k}, G^{s}\right)$ can be constructed as unions of subcollections of the finitely many sets $\mathcal{X}_{H_{1}}, \ldots, \mathcal{X}_{H_{r}}$.

Lemma 2.10 $\mathcal{X}_{H}$ is a closed subset of $\operatorname{Hom}\left(\mathbb{Z}^{k}, G^{s}\right)$ for all $H \subset G$.

It is worth noticing that in general the set of those $\rho \in \operatorname{Hom}\left(\mathbb{Z}^{k}, G^{s}\right)$ such that there is $g \in G$ with $g H^{-1}=\operatorname{Stab}_{G}(\rho)$ is neither open nor closed. 
Proof We prove that the complement of $\mathcal{X}_{H}$ in $\operatorname{Hom}\left(\mathbb{Z}^{k}, G^{s}\right)$ is open. Given $\rho \notin \mathcal{X}_{H}$, recall that $\operatorname{Stab}_{G}(\rho)$ is reductive and that $\operatorname{Orbit}_{G}(\rho)$ is Zariski closed by Theorem 2.7. Theorem 2.4 implies hence that $\rho$ belongs to a Zariski open set $U \subset \operatorname{Hom}\left(\mathbb{Z}^{k}, G^{s}\right)$ with the property that the stabilizer $\operatorname{Stab}_{G}\left(\rho^{\prime}\right)$ of any $\rho^{\prime} \in U$ is conjugate to a subgroup of $\operatorname{Stab}_{G}(\rho)$. The assumption that $H$ cannot be conjugated into $\operatorname{Stab}_{G}(\rho)$ then implies that neither can it be conjugated into $\operatorname{Stab}_{G}\left(\rho^{\prime}\right)$ for $\rho^{\prime} \in U \cap \operatorname{Hom}\left(\mathbb{Z}^{k}, G^{s}\right)$. This proves that $U \cap \mathcal{X}_{H}=\varnothing$. Since $U$ is Zariski open, it is a fortiori open; the claim follows.

Lemma 2.11 The sets $\operatorname{Hom}\left(\mathbb{Z}^{k}, K\right)$ and $\operatorname{Hom}\left(\mathbb{Z}^{k}, K^{G}\right)$ are closed in $\operatorname{Hom}\left(\mathbb{Z}^{k}, G^{s}\right)$.

Notice that $K^{G}$ is not a closed subset of $G$. This implies that $\operatorname{Hom}\left(\mathbb{Z}^{k}, K^{G}\right)$ is not closed in $\operatorname{Hom}\left(\mathbb{Z}^{k}, G\right)$.

Proof Clearly $\operatorname{Hom}\left(\mathbb{Z}^{k}, K\right)$ is closed because $K \subset G^{s}$ is compact and hence closed. Similarly, in order to prove that $\operatorname{Hom}\left(\mathbb{Z}^{k}, K^{G}\right)$ is closed in $\operatorname{Hom}\left(\mathbb{Z}^{k}, G^{s}\right)$, it suffices to show that $K^{G}$ is closed in $G^{s}$. Choosing an embedding of $G$ into $\mathrm{SL}_{n} \mathbb{C}$ for some suitable $n$, it suffices to prove that the closure of $K^{G} \subset \mathrm{SU}_{n}^{\mathrm{SL}_{n} \mathbb{C}}$ within the set of diagonalizable matrices consists of diagonalizable matrices that can be conjugated into $\mathrm{SU}_{n}$. However, a diagonalizable matrix $A \in \mathrm{SL}_{n} \mathbb{C}$ can be conjugated into $\mathrm{SU}_{n}$ if and only if all of its eigenvalues have unit norm; this is a closed condition (compare with Lemma 7.3).

Proposition 2.8 will follow easily from the fact that semialgebraic sets are triangulizable. Recall that a subset $X \subset \mathbb{C}^{N}$ is semialgebraic if it is the finite union of sets determined by a finite collection of real polynomial equalities and inequalities.

Theorem 2.12 Let $X$ be a semialgebraic set and $\left\{X_{i}\right\}_{i \in I}$ a locally finite collection of closed semialgebraic subsets of $X$. Then there is a simplicial complex $C$ and a homeomorphism $t: C \rightarrow X$ such that each $X_{i}$ is the image of a subcomplex of $C$.

Theorem 2.12 is a version of Whitney's Stratification Theorem [38; 39]; see also Thom [36]. We refer the interested reader to the PhD thesis of Kyle Hofmann [20] for a very pleasant discussion of these topics.

Lemma 2.13 The sets $\operatorname{Hom}\left(\mathbb{Z}^{k}, G^{s}\right), \operatorname{Hom}\left(\mathbb{Z}^{k}, K^{G}\right), \operatorname{Hom}\left(\mathbb{Z}^{k}, K\right)$ and $\mathcal{X}_{H}$ are semialgebraic. 
Proof Embedding $G$ into $\mathrm{SL}_{n} \mathbb{C}$ for some $n$, notice that $K \subset G$ is equal to the intersection of $G$ with a conjugate of $\mathrm{SU}_{n}$. It follows that $K$ is semialgebraic. Since $\operatorname{Hom}\left(\mathbb{Z}^{k}, K\right)$ is the intersection of the algebraic set $\operatorname{Hom}\left(\mathbb{Z}^{k}, G\right) \subset G^{k}$ with the semialgebraic set $K^{k}$, it is itself semialgebraic.

Let $G_{0}$ be the identity component of $G, \mathbb{T} \subset G_{0}$ a maximal torus and consider the following algebraic subvariety of $\left(G_{0} \times \mathbb{T}\right)^{k}$ :

$$
\mathcal{X}=\left\{\left(\left(g_{1}, a_{1}\right), \ldots,\left(g_{k}, a_{k}\right)\right) \in\left(G_{0} \times \mathbb{T}\right)^{k} \mid\left[g_{i} a_{i} g_{i}^{-1}, g_{j} a_{j} g_{j}^{-1}\right]=\operatorname{Id}_{G}\right\} .
$$

Notice that $\mathcal{X}$ is an affine variety and hence a semialgebraic set of $\mathbb{C}^{N}$ for some suitable choice of $N$. We consider now the map:

$$
\alpha: \mathcal{X} \rightarrow \operatorname{Hom}\left(\mathbb{Z}^{k}, G_{0}\right), \quad\left(g_{i}, a_{i}\right)_{i=1, \ldots, k} \mapsto\left(g_{i} a_{i} g_{i}^{-1}\right)_{i=1, \ldots, k} .
$$

By definition, $\alpha(\mathcal{X}) \subset \operatorname{Hom}\left(\mathbb{Z}^{k}, G_{0}^{s}\right)$. On the other hand, since $G_{0}$ is connected it follows that every semisimple element in $G_{0}$ can be conjugated into $\mathbb{T}$. This implies that $\alpha(\mathcal{X})=\operatorname{Hom}\left(\mathbb{Z}^{k}, G_{0}^{s}\right)$. We have proved that $\operatorname{Hom}\left(\mathbb{Z}^{k}, G_{0}^{s}\right)$ is the image of a semialgebraic set under a polynomial map. A renowned theorem of Tarski [35] asserts that images under polynomial maps of semialgebraic sets are semialgebraic. This proves that $\operatorname{Hom}\left(\mathbb{Z}^{k}, G_{0}^{s}\right)$ is semialgebraic. Let now $d$ be the index of $G_{0}$ in $G$ and consider the map:

$$
\operatorname{Hom}\left(\mathbb{Z}^{k}, G\right) \rightarrow \operatorname{Hom}\left(\mathbb{Z}^{k}, G_{0}\right), \quad\left(a_{1}, \ldots, a_{k}\right) \mapsto\left(a_{1}^{d}, \ldots, a_{k}^{d}\right) .
$$

The set $\operatorname{Hom}\left(\mathbb{Z}^{k}, G^{s}\right)$ is the preimage under this algebraic map of the semialgebraic set $\operatorname{Hom}\left(\mathbb{Z}^{k}, G_{0}^{s}\right)$ and hence is semialgebraic.

Notice now that the same argument shows that $\operatorname{Hom}\left(\mathbb{Z}^{k}, K^{G}\right)$ is also semialgebraic.

It remains to prove that $\mathcal{X}_{H}$ is semialgebraic for every subgroup $H \subset G$. To begin with, observe that

$$
\operatorname{Fix}^{s}(H)=\left\{\rho \in \operatorname{Hom}\left(\mathbb{Z}^{k}, G^{s}\right) \mid H \subset \operatorname{Stab}_{G}(\rho)\right\}
$$

is semialgebraic because it is the intersection of the fixed-point set of $H$ on $\operatorname{Hom}\left(\mathbb{Z}^{k}, G\right)$, an affine variety, with the semialgebraic set $\operatorname{Hom}\left(\mathbb{Z}^{k}, G^{s}\right)$. Consider the polynomial map:

$$
\beta: G \times \operatorname{Fix}^{s}(H) \rightarrow \operatorname{Hom}\left(\mathbb{Z}^{k}, G^{s}\right), \quad \beta:(g, \rho) \mapsto g \rho g^{-1} .
$$

It follows directly from its definition that $\mathcal{X}_{H}$ is equal to the image of $\beta$. Hence, Tarski's Theorem shows again that $\mathcal{X}_{H}$ is semialgebraic, as claimed.

Proposition 2.8 follows directly from Lemmas 2.9, 2.10, 2.11, 2.13 and Theorem 2.12. 


\subsection{The real case}

We consider now the real case. More concretely, assume that $G \subset \mathrm{SL}_{n} \mathbb{C}$ is a reductive algebraic group defined over $\mathbb{R}$ and denote by

$$
G(\mathbb{R})=\{g \in G \mid \bar{g}=g\}
$$

the group of real points, where $\bar{g}$ is the complex conjugate of $g$. We denote by $G(\mathbb{R})_{0}$ the identity component of the Lie group $G(\mathbb{R})$. Notice that $G(\mathbb{R})_{0}$ has finite index in $G_{0}(\mathbb{R})$.

The following lemma is one of the key facts needed to reduce the real case to the complex case.

Lemma 2.14 Let $G \subset \mathrm{SL}_{n} \mathbb{C}$ be the group of complex points of an algebraic group defined over $\mathbb{R}$ and $H \subset G$ an algebraic subgroup also defined over $\mathbb{R}$. There are finitely many $G(\mathbb{R})$-conjugacy classes of subgroups $H^{\prime} \subset G$ that are defined over $\mathbb{R}$ and conjugate to $H$ by an element in $G$.

The reader can find a proof of Lemma 2.14 in Serre [32, III 4.4]; for the sake of completeness we give a simple argument.

Proof Let $N=\mathcal{N}_{G}(H)$ be the normalizer of $H$ in $G$ and identify the smooth variety $G / N$ with the set of subgroups of $G$ conjugate to $H$. The subgroup $N$ is a closed subgroup defined over $\mathbb{R}$ and hence $G / N$ and the map $G \rightarrow G / N$ are also defined over $\mathbb{R}$; compare with [7, Chapter II].

Suppose that $H^{\prime} \in G / N$ and let $g \in G$ with $H^{\prime}=g H g^{-1}$. Taking into account that $\overline{H^{\prime}}=\bar{g} \bar{H} \bar{g}^{-1}=\bar{g} H \bar{g}^{-1}$ we deduce that the set of real points

$$
(G / N)(\mathbb{R})=\{g N \in G / N \mid \bar{g} N=g N\}
$$

of $G / N$ is exactly the set of subgroups of $G$ defined over $\mathbb{R}$ and conjugate to $H$ by an element in $G$.

Notice that the left action $G \curvearrowright G / N$ induces an action $G(\mathbb{R}) \curvearrowright(G / N)(\mathbb{R})$; the claim of Lemma 2.14 follows once we have proved that the latter action has only finitely many orbits. Taking into account that $(G / N)(\mathbb{R})$ is both a submanifold of $G / N$ and a real algebraic variety, and that as such, $(G / N)(\mathbb{R})$ has only finitely many connected components with respect to the Hausdorff topology [38], we deduce that it suffices to prove that $G(\mathbb{R}) \curvearrowright(G / N)(\mathbb{R})$ has open orbits.

To avoid introducing new notation, we will just prove that the $G(\mathbb{R})$-orbit of $H$ is open; the argument is identical for any other point of $(G / N)(\mathbb{R})$. Notice that the 
stabilizer of $H$ under the $G(\mathbb{R})$ action is the group $N(\mathbb{R})=N \cap G(\mathbb{R})$ of real points of $N$ and that the induced map

$$
G(\mathbb{R}) / N(\mathbb{R}) \rightarrow(G / N)(\mathbb{R})
$$

is locally injective. Observe also that the dimension of $(G / N)(\mathbb{R})$ as a manifold is at most as large as the complex dimension of $G / N$ :

$$
\operatorname{dim}_{\mathbb{R}}(G / N)(\mathbb{R}) \leq \operatorname{dim}_{\mathbb{C}} G / N .
$$

On the other hand, since $G$ and $N$ are groups defined over $\mathbb{R}$, we have

$$
\operatorname{dim}_{\mathbb{R}} G(\mathbb{R})=\operatorname{dim}_{\mathbb{C}} G \quad \text { and } \quad \operatorname{dim}_{\mathbb{R}} N(\mathbb{R})=\operatorname{dim}_{\mathbb{C}} N
$$

and hence

$$
\operatorname{dim}_{\mathbb{R}} G(\mathbb{R}) / N(\mathbb{R})=\operatorname{dim}_{\mathbb{C}} G / N \geq \operatorname{dim}_{\mathbb{R}}(G / N)(\mathbb{R}) .
$$

This implies that the locally injective map (2.4) is open.

The space $\operatorname{Hom}\left(\mathbb{Z}^{k}, G(\mathbb{R})\right)$ of representations of $\mathbb{Z}^{k}$ in $G(\mathbb{R})$ is the set of fixed points under complex conjugation in the variety $\operatorname{Hom}\left(\mathbb{Z}^{k}, G\right) \subset G \times \cdots \times G$; in particular, $\operatorname{Hom}\left(\mathbb{Z}^{k}, G(\mathbb{R})\right)$ is a real algebraic variety.

Notice that the action by conjugation $G \curvearrowright \operatorname{Hom}\left(\mathbb{Z}^{k}, G\right)$ induces the action by conjugation of $G(\mathbb{R})$ on $\operatorname{Hom}\left(\mathbb{Z}^{k}, G(\mathbb{R})\right)$. We prove that this action has finitely many orbit types of semisimple representations:

Proposition 2.6 (Real case) Let $G(\mathbb{R})$ be the set of real points of a reductive algebraic group defined over $\mathbb{R}$. The actions of $G(\mathbb{R})$ and $G(\mathbb{R})_{0}$ on $\operatorname{Hom}\left(\mathbb{Z}^{k}, G(\mathbb{R})\right.$ ) have only finitely many orbit types of semisimple representations.

Proof Consider the action $G \curvearrowright \operatorname{Hom}\left(\mathbb{Z}^{k}, G\right)$ by conjugation and observe that the stabilizer $\operatorname{Stab}_{G}(\rho)=\mathcal{Z}_{G}\left(\rho\left(\mathbb{Z}^{k}\right)\right)$ of any point $\rho \in \operatorname{Hom}\left(\mathbb{Z}^{k}, G(\mathbb{R})\right)$ is a closed subgroup defined over $\mathbb{R}$. By Proposition 2.6, the set of all stabilizers of semisimple points in $\operatorname{Hom}\left(\mathbb{Z}^{k}, G(\mathbb{R})\right)$ falls into finitely many $G_{0}$-conjugacy classes. Lemma 2.14 implies now that they also fall into finitely many $G(\mathbb{R})$-conjugacy classes, as we needed to prove. Since $G(\mathbb{R})_{0}$ has finite index in $G(\mathbb{R})$, the same is true for the action $G(\mathbb{R})_{0} \curvearrowright \operatorname{Hom}\left(\mathbb{Z}^{k}, G(\mathbb{R})\right)$.

Continuing with the same notation, let $G(\mathbb{R})^{s}=G^{s} \cap G(\mathbb{R})$ be the set of real semisimple elements and consider

$$
\operatorname{Hom}\left(\mathbb{Z}^{k}, G(\mathbb{R})^{s}\right)=\left\{\left(a_{1}, \ldots, a_{k}\right) \in \operatorname{Hom}\left(\mathbb{Z}^{k}, G\right) \mid a_{i} \in G(\mathbb{R})^{s} \forall i\right\},
$$


the set of real semisimple representations. As the fixed point set under complex conjugation, $\operatorname{Hom}\left(\mathbb{Z}^{k}, G(\mathbb{R})^{s}\right)$ is closed semialgebraic subset of $\operatorname{Hom}\left(\mathbb{Z}^{k}, G^{s}\right)$. This said, one can apply word-for-word the arguments used to prove Proposition 2.8 to show:

Proposition 2.8 (Real case) Suppose that $G(\mathbb{R})$ is the group of real points of a reductive algebraic group defined over $\mathbb{R}$, and let $K(\mathbb{R}) \subset G(\mathbb{R})$ be a maximal compact subgroup. There is a triangulation of $\operatorname{Hom}\left(\mathbb{Z}^{k}, G(\mathbb{R})^{s}\right)$ such that

$$
\mathcal{X}_{H}=\left\{\rho \in \operatorname{Hom}\left(\mathbb{Z}^{k}, G(\mathbb{R})^{s}\right) \mid \exists g \in G(\mathbb{R}) \text { with } g H g^{-1} \subset \operatorname{Stab}_{G(\mathbb{R})}(\rho)\right\}
$$

is a subcomplex for every subgroup $H \subset G(\mathbb{R})$. Moreover, $\operatorname{Hom}\left(\mathbb{Z}^{k}, K(\mathbb{R})\right)$ and $\operatorname{Hom}\left(\mathbb{Z}^{k}, K(\mathbb{R})^{G(\mathbb{R})}\right)$ are also subcomplexes.

\section{Well-centered groups}

Throughout this section we assume that $G$ is the group of complex points of a reductive algebraic group and that $K \subset G$ is a maximal compact subgroup; the real case will only be discussed at the end. Recall that if $H \subset G$ is a subgroup, then $\mathcal{Z}_{G}(H)$ is the centralizer of $H$ in $G$. We denote by $C(H)$ the center of $H$.

\subsection{Diagonalizable groups}

A Zariski closed subgroup $A \subset G$ is diagonalizable if it is abelian and consists of semisimple elements. Notice that the Zariski closure of every abelian subgroup consisting of semisimple elements is diagonalizable. Also, the center of a reductive group $C(G)$ is diagonalizable. Conversely, the centralizer $\mathcal{Z}_{G}(A)$ of a diagonalizable subgroup $A \subset G$ is reductive.

We refer the reader to [7, Chapter 8] for basic facts on diagonalizable groups; we will just prove a few surely well-known facts for which we have not found a reference.

Lemma 3.1 Suppose that $G \subset \mathrm{SL}_{n} \mathbb{C}$ is a reductive algebraic group and that $A \subset G$ is a diagonalizable subgroup. Then:

- $A$ contains a unique maximal compact subgroup $\bar{A}$. Moreover, $\bar{A}$ is Zariski dense in $A$.

- $A$ contains a Zariski dense subgroup generated by $n$ elements. 
Proof Denote by $A_{0}$ the connected component of the identity so that we have an exact sequence

$$
0 \rightarrow A_{0} \rightarrow A \rightarrow A / A_{0} \rightarrow 0,
$$

where $A / A_{0}$ is a finite abelian group. This exact sequence splits [7, 8.7], and hence $A$ is the direct product of the torus $A_{0}$ and a finite subgroup $F$ of $G$ isomorphic to $A / A_{0}$. Identifying $A_{0}$ with $\mathbb{C}^{*} \times \cdots \times \mathbb{C}^{*}$ we obtain that

$$
\bar{A}=\mathbb{S}^{1} \times \cdots \times \mathbb{S}^{1} \times F
$$

is a maximal compact group that is clearly Zariski dense. Given any $\left(a_{0}, f\right) \in A_{0} \times$ $F=A$, consider the powers $\left(a_{0}, f\right)^{n|F|}=\left(a_{0}^{n|F|}, 1\right)$, where $|F|$ is the order of the finite group $F$. It follows that if $\left(a_{0}, f\right)$ belongs to some compact subgroup, then $a_{0} \in \mathbb{S}^{1} \times \cdots \times \mathbb{S}^{1}$. This proves that the maximal compact subgroup $\bar{A}$ is unique, and the first claim is proved.

Starting now with the proof of the second claim, observe that the finite subgroup $F \subset G \subset \mathrm{SL}_{n} \mathbb{C}$ is contained in an $\mathrm{SL}_{n} \mathbb{C}$-conjugate of the group of diagonal matrices with determinant one. It follows that $F$ is isomorphic to a subgroup of $\mathbb{Z} / q_{1} \mathbb{Z} \times \cdots \times$ $\mathbb{Z} / q_{n-1} \mathbb{Z}$ with $q_{i} \in \mathbb{Z}$. In particular, $F$ can be generated by $n-1$ elements. Since the torus $A_{0}$ contains a Zariski dense cyclic subgroup, it follows that $A$ has a Zariski dense subgroup generated by $n$ elements.

Suppose that $\left(a_{1}, \ldots, a_{k}\right) \in \operatorname{Hom}\left(\mathbb{Z}^{k}, K^{G}\right)$ and recall that this just means that each $a_{i}$ can be conjugated into $K$. Since $K$ consists of semisimple elements, Lemma 2.5 implies that the Zariski closure $A \subset G$ of the group generated by $a_{1}, \ldots, a_{k}$ is a diagonalizable subgroup of $G$. Each $a_{i}$ belongs to some compact subgroup of $A$ and by Lemma 3.1, $A$ contains a unique maximal compact subgroup $\bar{A}$; hence $a_{i} \in \bar{A}$ for all $i$. Since $\bar{A}$ can be conjugated into $K$, we have proven that the elements $a_{i}$ can be simultaneously conjugated into $K$.

Lemma 3.2 An element $\rho \in \operatorname{Hom}\left(\mathbb{Z}^{k}, G\right)$ belongs to $\operatorname{Hom}\left(\mathbb{Z}^{k}, K^{G}\right)$ if and only if there is $g \in G$ with $g \rho\left(\mathbb{Z}^{k}\right) g^{-1} \subset K$.

\subsection{Trans}

Given two subsets $X_{1}, X_{2}$ of $G$, we denote by

$$
\operatorname{Trans}_{G}\left(X_{1}, X_{2}\right)=\left\{g \in G \mid g X_{1} g^{-1} \subset X_{2}\right\}
$$

the subset of $G$ consisting of all elements that conjugate $X_{1}$ into $X_{2}$. Notice for instance that if $H$ is a subgroup of $G$, then

$$
\operatorname{Trans}_{G}(H, H)=\mathcal{N}_{G}(H)
$$


is the normalizer of $H$ in $G$. Abusing notation, we will denote the intersection of $\operatorname{Trans}_{G}(\cdot, \cdot)$ with the identity component $G_{0}$ of $G$ by

$$
\operatorname{Trans}_{G_{0}}\left(X_{1}, X_{2}\right)=\operatorname{Trans}_{G}\left(X_{1}, X_{2}\right) \cap G_{0},
$$

and accordingly:

$$
\begin{aligned}
& \mathcal{Z}_{G_{0}}(H)=\mathcal{Z}_{G}(H) \cap G_{0}, \\
& \mathcal{N}_{G_{0}}(H)=\mathcal{N}_{G}(H) \cap G_{0} .
\end{aligned}
$$

The sets $\operatorname{Trans}_{K}(\cdot, \cdot)$ and $\operatorname{Trans}_{K_{0}}(\cdot, \cdot)$ are defined accordingly.

Suppose that $A, A^{\prime} \subset G$ are diagonalizable. It is easy to see that $\operatorname{Trans}_{G}\left(A, A^{\prime}\right)$ is an algebraic variety [7, Chapter I] and hence has finitely many connected components. We show now that each one of them is homeomorphic to the connected component of the identity in the centralizer $\mathcal{Z}_{G}(A)$ of $A$ in $G$.

Lemma 3.3 Suppose that $A, A^{\prime}$ are diagonalizable subgroups of $G$, and let $V$ be a connected component of $\operatorname{Trans}_{G}\left(A, A^{\prime}\right)$. For all $g_{0} \in V$, the map

$$
\mathcal{Z}_{G}(A)_{0} \rightarrow V, \quad h \mapsto g_{0} h
$$

is an isomorphism. Here, $\mathcal{Z}_{G}(A)_{0}$ is the identity component of the centralizer of $A$ in $G$.

In particular, the Weyl groups $W_{A}=\mathcal{N}_{G}(A) / \mathcal{Z}_{G}(A)$ and $W_{A}^{0}=\mathcal{N}_{G_{0}}(A) / \mathcal{Z}_{G_{0}}(A)$ are finite for every diagonalizable subgroup $A$ of $G$.

Proof With the same notation as in the statement of the lemma, observe that by Lemma 3.1 we have

$$
\operatorname{Trans}_{G}\left(A, A^{\prime}\right)=\operatorname{Trans}_{G}\left(\bar{A}, \bar{A}^{\prime}\right),
$$

where $\bar{A}, \bar{A}^{\prime}$ are unique maximal compact subgroups of $A$ and $A^{\prime}$. Endowed with the compact open topology, $\operatorname{Hom}\left(\bar{A}, \bar{A}^{\prime}\right)$ is a totally disconnected space. Hence, the map

$$
\operatorname{Trans}\left(A, A^{\prime}\right) \rightarrow \operatorname{Hom}\left(A, A^{\prime}\right), \quad g \mapsto\left\{a \mapsto g a g^{-1}\right\}
$$

is constant on each connected component (compare with $[7,8.10]$ ). In other words, it follows that

$$
g a g^{-1}=g_{0} a\left(g_{0}\right)^{-1}
$$

for all $a \in A$ and all $g \in V$. This implies in particular that $g_{0}^{-1} g \in \mathcal{Z}_{G}(A)_{0}$ for all $g \in V$. Since on the other hand the morphism

$$
\mathcal{Z}_{G}(A)_{0} \rightarrow V, \quad h \mapsto g_{0} h
$$

is injective, the claim follows. 
The following result, of capital importance in this paper, relates $\operatorname{Trans}_{G}$ and $\operatorname{Trans}_{K}$, where $K \subset G$ is a maximal compact subgroup.

Lemma 3.4 If $X_{1}, X_{2} \subset K$ are subsets of $K$, then the inclusion

$$
\operatorname{Trans}_{K}\left(X_{1}, X_{2}\right) \hookrightarrow \operatorname{Trans}_{G}\left(X_{1}, X_{2}\right)
$$

is a homotopy equivalence. In particular we have:

(1) $X_{1}, X_{2} \subset K$ are conjugate in $G$ if and only if they are in $K$.

(2) If $H \subset K$ is a subgroup then the inclusion $\mathcal{N}_{K}(H) \hookrightarrow \mathcal{N}_{G}(H)$ is a homotopy equivalence.

Before proving Lemma 3.4 we need to recall a few facts about the Cartan decomposition. It is well-known that the Lie algebra $\mathfrak{g}$ of the reductive group $G$ contains an $\operatorname{Ad}(K)$ invariant linear subspace $\mathfrak{p} \subset \mathfrak{g}$ such that the map

$$
\mathfrak{p} \times K \rightarrow G, \quad(X, k) \mapsto e^{X} k
$$

is a diffeomorphism. In particular, we can write every element $g \in G$ uniquely as $g=e^{X_{g}} k_{g}$, where $X_{g} \in \mathfrak{p}$ and $k_{g} \in K$; this is the Cartan decomposition of $g$ associated to $K$.

Lemma 3.5 Suppose that $k \in K$ and $g=e^{X_{g}} k_{g} \in G$ are such that $g k g^{-1} \in K$. Then $k_{g} k k_{g}^{-1}=g k g^{-1}$ and $\operatorname{Ad}\left(g k g^{-1}\right)\left(X_{g}\right)=X_{g}$.

Proof We have $e^{X_{g}} k_{g} k k_{g}^{-1} e^{-X_{g}}=g k g^{-1}=k^{\prime} \in K$ and hence that:

$$
e^{X_{g}} k_{g} k k_{g}^{-1}=k^{\prime} e^{X_{g}}=e^{\operatorname{Ad}\left(k^{\prime}\right)\left(X_{g}\right)} k^{\prime} .
$$

The claim follows now from the uniqueness of the Cartan decomposition.

Proof of Lemma 3.4 By Lemma 3.5 we have for $g=e^{X} k \in G$ that $g X_{1} g^{-1}=X_{2}$ if and only if $k X_{1} k^{-1}=X_{2}$ and $X \in \operatorname{Fix}\left(\operatorname{Ad}\left(X_{2}\right)\right)=\left\{X \in \mathfrak{p} \mid \operatorname{Ad}(g)(X)=X \forall g \in X_{2}\right\}$. It follows that there is a diffeomorphism:

$$
\operatorname{Trans}_{G}\left(X_{1}, X_{2}\right) \simeq \operatorname{Trans}_{K}\left(X_{1}, X_{2}\right) \times \operatorname{Fix}\left(\operatorname{Ad}\left(X_{2}\right)\right) .
$$

Notice that $\operatorname{Fix}\left(\operatorname{Ad}\left(X_{2}\right)\right)$ is contractible because it is a linear subspace of $\mathfrak{p}$. It follows that the inclusion

$$
\operatorname{Trans}_{K}\left(X_{1}, X_{2}\right) \rightarrow \operatorname{Trans}_{G}\left(X_{1}, X_{2}\right)
$$

is a homotopy equivalence. 
Remark With the notation of Lemma 3.5, let $G_{0}$ and $K_{0}$ be, respectively, the identity connected components of $G$ and $K$. Notice that $g \in G_{0}$ if and only if $k_{g} \in K_{0}$. In particular, the argument given to prove Lemma 3.4 also shows that the inclusion

$$
\operatorname{Trans}_{K_{0}}\left(X_{1}, X_{2}\right) \rightarrow \operatorname{Trans}_{G_{0}}\left(X_{1}, X_{2}\right)
$$

is a homotopy equivalence for any two subsets $X_{1}, X_{2} \subset K$.

\subsection{Well-centered subgroups}

We will be interested in a particular class of diagonalizable subgroups of the reductive group $G$ and its maximal compact subgroup $K$.

A diagonalizable subgroup $A \subset G$ is well-centered in $G$ if it is the center of its centralizer: $A=C\left(\mathcal{Z}_{G}(A)\right)$. Similarly, an abelian subgroup $H \subset K$ is well-centered in $K$ if $H=C\left(\mathcal{Z}_{K}(H)\right)$. Recall that the Zariski closure in $G$ of every abelian semisimple subgroup is diagonalizable.

Well-centered groups will play a central role in this paper; we discuss here briefly what they are in the particular case that $G=\mathrm{SL}_{n} \mathbb{C}$.

Example: well-centered groups in $\mathbf{S L}_{\boldsymbol{n}} \mathbb{C}$ It is well-known that every diagonalizable subgroup $A$ of $\mathrm{SL}_{n} \mathbb{C}$ is "diagonalizable", meaning that there is a direct sum decomposition

$$
\mathbb{C}^{n}=V_{1} \oplus \cdots \oplus V_{r}
$$

such that every element $a \in A$ preserves each one of the factors $V_{i}$ and acts on it by a homothety. In fact, taking the direct sum decomposition (3.1) with the smallest possible number of factors, we have the following description of the centralizer of $A$ :

$$
\mathcal{Z}_{\mathrm{SL}_{n} \mathbb{C}}(A)=\mathrm{SL}_{n} \mathbb{C} \cap\left(\mathrm{GL}\left(V_{1}\right) \times \cdots \times \mathrm{GL}\left(V_{r}\right)\right) .
$$

We might refer to the unique direct sum decomposition satisfying (3.2) as the canonical direct sum decomposition diagonalizing $A$; notice that its factors are not ordered.

The center of the centralizer of $A$ is then the subgroup of $\mathrm{SL}_{n} \mathbb{C}$ consisting of all elements diagonalized by the canonical direct sum decomposition (3.1) associated to $A$ :

$$
C\left(\mathcal{Z}_{\mathrm{SL}_{n} \mathbb{C}}(A)\right)=\left\{g \in \mathrm{SL}_{n} \mathbb{C} \mid \forall i=1, \ldots, r \exists \lambda_{i} \text { with } g v_{i}=\lambda_{i} v_{i} \forall v_{i} \in V_{i}\right\}
$$

From this discussion we deduce that:

There is a natural correspondence between the sets of well-centered groups in $\mathrm{SL}_{n} \mathbb{C}$ and of unordered direct sum decompositions of $\mathbb{C}^{n}$. 
Returning now to the general setting, we state for further reference a few general facts about well-centered groups:

\section{Lemma 3.6}

(1) We have $C\left(\mathcal{Z}_{G}(A)\right)=\mathcal{Z}_{G}\left(\mathcal{Z}_{G}(A)\right)$ for any $A \subset G$ abelian.

(2) Suppose that $A_{1}$ and $A_{2}$ are well-centered groups in $G$. Then $A_{1} \supset A_{2}$ if and only if $\mathcal{Z}_{G}\left(A_{1}\right) \subset \mathcal{Z}_{G}\left(A_{2}\right)$.

(3) Suppose that $A \subset G$ is an abelian subgroup consisting of semisimple elements. Then $\hat{A}=C\left(\mathcal{Z}_{G}(A)\right)$ is the smallest well-centered group containing $A$.

The proof of Lemma 3.6 is a straightforward computation in elementary group theory; notice only that in (3), the proviso that $A$ consist of semisimple elements is just to ensure that $\hat{A}$ is diagonalizable. We leave the proof of Lemma 3.6 to the reader.

The following is the central observation of this section:

Lemma 3.7 Suppose that $H \subset K$ is well-centered in $K$. Then $H$ is the unique maximal compact subgroup in the well-centered group $C\left(\mathcal{Z}_{G}(H)\right)$. In particular

$$
H=K \cap C\left(\mathcal{Z}_{G}(H)\right)
$$

is Zariski dense in $C\left(\mathcal{Z}_{G}(H)\right)$.

Proof By Lemma 3.6, $\hat{H}=C\left(\mathcal{Z}_{G}(H)\right)$ is well-centered and in particular diagonalizable. Lemma 3.1 implies hence that $\hat{H}$ has a unique maximal compact subgroup $\bar{H} \subset \hat{H}$. Since $\bar{H}$ is also Zariski dense, by Lemma 3.1 it will suffice to prove that $H=\bar{H}$. To begin with, notice that

$$
H \subset \bar{H} \text {. }
$$

We have to prove the opposite inclusion. Since $\bar{H}$ is compact, there is $g=e^{X} k \in G$ with $g \bar{H} g^{-1} \subset K$. By Lemma 3.5 we have $g h g^{-1}=k h k^{-1}$ for all $h \in H$. So, replacing $g$ by $k^{-1} g$, we may assume that $g \in \mathcal{Z}_{G}(H)$. Now, since $\hat{H}$ is the center of $\mathcal{Z}_{G}(H)$ we have that $g \hat{H} g^{-1}=\hat{H}$. By uniqueness of the maximal compact subgroup $\bar{H}$ of $\hat{H}$, we have $g \bar{H} g^{-1}=\bar{H}$. But this proves that $\bar{H} \subset K$. Hence

$$
\bar{H} \subset K \cap \mathcal{Z}_{G}(H)=\mathcal{Z}_{K}(H) .
$$

Now, $\bar{H}$ is central in $\mathcal{Z}_{G}(H)$ and hence a fortiori in $\mathcal{Z}_{K}(H)$. This shows that

$$
\bar{H} \subset C\left(\mathcal{Z}_{K}(H)\right)=H .
$$

This proves the claim. 
Example: well-centered groups in $\mathbf{S L}_{\boldsymbol{n}} \mathbb{C}$ and polynomials As a matter of example, we discuss the third claim of Lemma 3.6 for $G=\mathrm{SL}_{n} \mathbb{C}$ and $A$ generated by a single semisimple element $a \in \mathrm{SL}_{n} \mathbb{C}$. Denote by (3.1) the canonical direct sum decomposition associated to the Zariski closure of $A=\langle a\rangle$ and consider

$$
\mathcal{A}=\left\{g \in M_{n} \mathbb{C} \mid \forall i=1, \ldots, r \exists \lambda_{i} \text { with } g v_{i}=\lambda_{i} v_{i} \forall v_{i} \in V_{i}\right\},
$$

ie, the linear subspace of the vector space of all $n \times n$ matrices which are diagonalized by (3.1). The space $\mathcal{A}$ has dimension $r$ and the Vandermonde determinant implies that the elements $\mathrm{Id}, a, a^{2}, \ldots, a^{r-1} \in \mathcal{A}$ are linearly independent. It follows that $\mathcal{A}$ can be identified with the subring $\mathbb{C}[a]$ of $M_{n} \mathbb{C}$ generated by $a$. From (3.3) we deduce hence that

$$
C\left(\mathcal{Z}_{\mathrm{SL}_{n} \mathbb{C}}(a)\right)=\mathbb{C}[a] \cap \mathrm{SL}_{n} \mathbb{C} .
$$

Taking into account that $\mathcal{Z}_{G}(a)=\mathcal{Z}_{\mathrm{SL}_{n} \mathbb{C}}(a) \cap G$ for every subgroup $G$ of $\mathrm{SL}_{n} \mathbb{C}$ we deduce:

Lemma 3.8 Suppose that $G \subset \mathrm{SL}_{n} \mathbb{C}$ is an algebraic group and that $a \in G$ is semisimple. Then $C\left(\mathcal{Z}_{G}(a)\right)=\mathbb{C}[a] \cap G$ is the smallest well-centered group containing $a$.

\subsection{Manifolds of well-centered groups}

Continuing with the same notation, let

$$
\begin{aligned}
& \mathcal{G}=\{A \subset G \mid A \text { well-centered in } G\}, \\
& \mathcal{K}=\{H \subset K \mid H \text { well-centered in } K\} .
\end{aligned}
$$

The groups $G$ and $K$ act, from the left, on $\mathcal{G}$ and $\mathcal{K}$ by conjugacy.

Lemma 3.9 Let $G_{0}$ and $K_{0}$ be the identity components of $G$ and $K$, respectively. The actions $G_{0} \curvearrowright \mathcal{G}$ and $K_{0} \curvearrowright \mathcal{K}$ have finitely many orbits.

Proof By Lemma 3.1, there is some fixed $n$ such that every well-centered subgroup $A$ of $G$ contains a Zariski dense subgroup $B$ generated by $n$ elements. Observe that $A$ and $B$ have the same centralizer. Since $B$ is generated by $n$ elements, there is a semisimple representation $\mathbb{Z}^{n} \rightarrow G$ with image $B$. By Proposition 2.6, there are only finitely many $G_{0}$-conjugacy classes of centralizers of images of semisimple representations $\mathbb{Z}^{n} \rightarrow G$. This proves that there are finitely many $G_{0}$-conjugacy classes of centralizers of well-centered groups. 
Suppose now that $A$ and $A^{\prime}$ have $G_{0}$-conjugate centralizers. Then we have

$$
A^{\prime}=C\left(\mathcal{Z}_{G}\left(A^{\prime}\right)\right)=C\left(g \mathcal{Z}_{G}(A) g^{-1}\right)=g\left(C\left(\mathcal{Z}_{G}(A)\right)\right) g^{-1}=g A g^{-1} .
$$

It follows that $A$ and $A^{\prime}$ belong to the same orbit of $G_{0}$ under conjugation. We have proved that $G_{0} \curvearrowright \mathcal{G}$ has only finitely many orbits.

The claim for $K_{0} \curvearrowright \mathcal{K}$ follows from the validity of the claim for $G_{0} \curvearrowright \mathcal{G}$ and Lemma 3.4, the remark following the proof of Lemma 3.4, and Lemma 3.7.

Give $\mathcal{G}$ the topology in which each $G_{0}$-orbit has the obvious structure as a connected $G_{0}$-homogenous manifold. More precisely, we consider the connected component of $\mathcal{G}$ to which some well-centered group $A$ belongs as a smooth manifold via the canonical identification as a $G_{0}$-set with the homogenous space $G_{0} / \mathcal{N}_{G_{0}}(A)$; here $\mathcal{N}_{G_{0}}(A)$ is the intersection of $G_{0}$ with the normalizer $\mathcal{N}_{G}(A)$ of $A$ in $G$. Having a topology on each $G_{0}$-orbit we endow $\mathcal{G}$ with the disjoint union topology.

Similarly, we consider $\mathcal{K}$ with the topology in which each $K_{0}$-orbit is a connected $K_{0}$-homogenous manifold.

Example: manifolds of well-centered groups in $\mathbf{S L}_{\boldsymbol{n}} \mathbb{C}$ As mentioned above, there is a natural correspondence between the sets of well-centered groups in $\mathrm{SL}_{n} \mathbb{C}$ and of unordered direct sum decompositions of $\mathbb{C}^{n}$. In particular, the manifolds of wellcentered groups in $\mathrm{SL}_{n} \mathbb{C}$ are disjoint unions of quotients of products of open sets in Grassmannians. Similarly, the manifolds of well-centered groups in $\mathrm{SU}_{n}$ are disjoint unions of quotients of compact flag manifolds.

From the proof of Lemma 3.9 we deduce that two well-centered groups $A, A^{\prime} \in \mathcal{G}$ are in the same connected component of $\mathcal{G}$ if and only if they, or equivalently their centralizers, are $G_{0}$-conjugate. We state this fact for later reference:

Lemma 3.10 For any two well-centered groups $A, A^{\prime} \in \mathcal{G}$ the following are equivalent:

- $A$ and $A^{\prime}$ are in the same connected component of $\mathcal{G}$.

- $A$ and $A^{\prime}$ are conjugate by an element in $G_{0}$.

- $\mathcal{Z}_{G}(A)$ and $\mathcal{Z}_{G}\left(A^{\prime}\right)$ are conjugate by an element in $G_{0}$.

We come now to the main result of this section:

Proposition 3.11 The map $\iota: \mathcal{K} \rightarrow \mathcal{G}, \iota(H)=C\left(\mathcal{Z}_{G}(H)\right)$, is an injective homotopy equivalence. 
Proof Suppose that $H, H^{\prime} \in \mathcal{K}$ are such that $\iota(H)=\iota\left(H^{\prime}\right)$. By Lemma 3.7, $H$ and $H^{\prime}$ are each the unique maximal compact subgroup of the well-centered group $\iota(H)=\iota\left(H^{\prime}\right)$; in other words, $H=H^{\prime}$. This proves that $\iota$ is injective.

We prove next that $\iota$ induces a bijection between the connected components of $\mathcal{K}$ and $\mathcal{G}$. Suppose that $H, H^{\prime} \in \mathcal{K}$ are such that $\iota(H)$ and $\iota\left(H^{\prime}\right)$ belong to the same connected component of $\mathcal{G}$. In other words, there is $g \in G_{0}$ with $\iota\left(H^{\prime}\right)=g_{\iota}(H) g^{-1}$. We deduce again from Lemma 3.7 that $H^{\prime}=\mathrm{gHg}^{-1}$. The remark following the proof of Lemma 3.4 implies then that $H$ and $H^{\prime}$ are $K_{0}$-conjugate as well. This shows that $H$ and $H^{\prime}$ belong to the same connected component of $\mathcal{K}$. We have proven that the map between connected components is injective. Given now $A \in \mathcal{G}$, let $\bar{A}$ be the unique maximal compact subgroup of $A$, and let $g \in G_{0}$ be such that $g \bar{A} g^{-1} \subset K$. Since $g \bar{A} g^{-1}$ is the unique maximal compact subgroup of the well-centered group $g \mathrm{Ag}^{-1}$, it follows from Lemma 3.7 that $g \bar{A} g^{-1} \in \mathcal{K}$ and that

$$
\iota\left(g \bar{A} g^{-1}\right)=g A g^{-1} \text {. }
$$

Since $A$ and $g \mathrm{Ag}^{-1}$ belong to the same connected component of $\mathcal{G}$ we deduce that the map between the sets of connected components is also surjective.

It remains to prove that the restriction of $\iota$ to each connected component $\mathcal{K}_{0} \subset \mathcal{K}$ is a homotopy equivalence into the corresponding component $\mathcal{G}_{0} \subset \mathcal{G}$. Fix $H \in \mathcal{K}_{0}$ and let $A=\iota(H) \in \mathcal{G}_{0}$. By definition we have that $\mathcal{G}_{0}=\operatorname{Orbit}_{G_{0}}(A)$, that $\mathcal{K}_{0}=\operatorname{Orbit}_{K}(H)$, and that

$$
\operatorname{Stab}_{K_{0}}(H)=\mathcal{N}_{K_{0}}\left(H_{0}\right), \quad \operatorname{Stab}_{G_{0}}(A)=\mathcal{N}_{G_{0}}(A)=\mathcal{N}_{G_{0}}(H),
$$

where the last equality holds by Lemma 3.1 and Lemma 3.7.

The remark following Lemma 3.4 implies that the inclusion $\operatorname{Stab}_{K_{0}}(H) \rightarrow \operatorname{Stab}_{G_{0}}(A)$ is a homotopy equivalence. In particular we have a diagram as follows

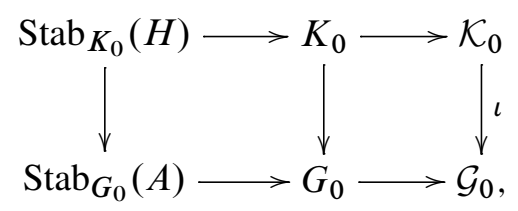

where the rows are fibrations and where the first two vertical arrows are homotopy equivalences. It follows that the third vertical arrow is also a homotopy equivalence, as claimed. 


\subsection{Orderings on $\pi_{0}(\mathcal{G})$ and $\pi_{0}(\mathcal{K})$}

We introduce a partial order on the set $\pi_{0}(\mathcal{G})$ of connected components of $\mathcal{G}$ as follows: if $\mathcal{G}_{1}$ and $\mathcal{G}_{2}$ are connected components of $\mathcal{G}$ then we say that

$$
\mathcal{G}_{1} \geq \mathcal{G}_{2}
$$

if for some, and hence for every, $A_{1} \in \mathcal{G}_{1}$ there is $A_{2} \in \mathcal{G}_{2}$ with $A_{1} \supset A_{2}$. Recall that Lemma 3.6 implies that this is equivalent to asking that for every $A_{1} \in \mathcal{G}_{1}$ there is $A_{2} \in \mathcal{G}_{2}$ with $\mathcal{Z}_{G}\left(A_{1}\right) \subset \mathcal{Z}_{G}\left(A_{2}\right)$.

On $\pi_{0}(\mathcal{K})$ we consider the analogously defined partial ordering: if $\mathcal{K}_{1}$ and $\mathcal{K}_{2}$ are connected components of $\mathcal{K}$, then we say that

$$
\mathcal{K}_{1} \geq \mathcal{K}_{2}
$$

if for some, and hence for every, $H_{1} \in \mathcal{K}_{1}$, there is $H_{2} \in \mathcal{K}_{2}$ with $H_{1} \supset H_{2}$. Lemmas 3.1, 3.6 and 3.7 imply that, as above, this is equivalent to asking that for every $H_{1} \in \mathcal{K}_{1}$ there is a $H_{2} \in \mathcal{K}_{2}$ with $\mathcal{Z}_{G}\left(H_{1}\right) \subset \mathcal{Z}_{G}\left(H_{2}\right)$.

The following claim follows directly from the definitions:

Lemma 3.12 The homotopy equivalence $\iota: \mathcal{K} \rightarrow \mathcal{G}$ induces an order-preserving bijection $\pi_{0}(\iota): \pi_{0}(\mathcal{K}) \rightarrow \pi_{0}(\mathcal{G})$.

Henceforth we will identify $\mathcal{K}$ and $\iota(\mathcal{K})$ and so feel free to write $\mathcal{K} \subset \mathcal{G}$.

Example: the orderings if $\boldsymbol{G}=\mathrm{SL}_{\boldsymbol{n}} \mathbb{C}$ As mentioned above, there is a natural correspondence between the manifolds of well-centered groups in $\mathrm{SL}_{n} \mathbb{C}$ and of unordered direct sum decompositions of $\mathbb{C}^{n}$. If $\mathcal{G}_{1}$ and $\mathcal{G}_{2}$ are connected components of the manifolds of unordered direct sum decompositions then $\mathcal{G}_{1} \geq \mathcal{G}_{2}$ if and only if for every direct sum decomposition $\mathbb{C}^{n}=C_{1} \oplus \cdots \oplus V_{r}$ in $\mathcal{G}_{1}$ there is a direct sum decomposition $\mathbb{C}^{n}=W_{1} \oplus \cdots \oplus W_{s}$ in $\mathcal{G}_{2}$ such that for each $i \in\{1, \ldots, r\}$ there is $j \in\{1, \ldots, s\}$ with $V_{i} \subset V_{j}$.

\subsection{The set $\mathcal{R}_{\mathcal{G}_{1}, \mathcal{G}_{2}}$}

Suppose that we have two components $\mathcal{G}_{1}, \mathcal{G}_{2}$ of $\mathcal{G}$ with $\mathcal{G}_{1} \geq \mathcal{G}_{2}$ and consider the set

$$
\mathcal{R}_{\mathcal{G}_{1}, \mathcal{G}_{2}}=\left\{\left(A_{1}, A_{2}\right) \in \mathcal{G}_{1} \times \mathcal{G}_{2} \mid A_{1} \supset A_{2}\right\} .
$$

Our next goal is to prove that the projections of $\mathcal{R}_{\mathcal{G}_{1}, \mathcal{G}_{2}}$ onto $\mathcal{G}_{1}$ and $\mathcal{G}_{2}$ are respectively a finite cover and a locally trivial fibration. Before doing so, notice that 
for any $\left(A_{1}, A_{2}\right) \in \mathcal{R}_{\mathcal{G}_{1}, \mathcal{G}_{2}}$ the groups $\mathcal{N}_{G_{0}}\left(A_{1}\right), \mathcal{N}_{G_{0}}\left(A_{2}\right)$ act from the right on $\operatorname{Trans}_{G_{0}}\left(A_{2}, A_{1}\right)$ :

$$
\begin{array}{ll}
\operatorname{Trans}_{G_{0}}\left(A_{2}, A_{1}\right) \times \mathcal{N}_{G_{0}}\left(A_{2}\right) \rightarrow \operatorname{Trans}_{G_{0}}\left(A_{2}, A_{1}\right), & (g, h) \mapsto g h, \\
\operatorname{Trans}_{G_{0}}\left(A_{2}, A_{1}\right) \times \mathcal{N}_{G_{0}}\left(A_{1}\right) \rightarrow \operatorname{Trans}_{G_{0}}\left(A_{2}, A_{1}\right), & (g, h) \mapsto h^{-1} g .
\end{array}
$$

We prove:

Lemma 3.13 Fixing $\left(A_{1}, A_{2}\right) \in \mathcal{R}_{\mathcal{G}_{1}, \mathcal{G}_{2}}$ the following holds:

- The projection $p_{2}: \mathcal{R}_{\mathcal{G}_{1}, \mathcal{G}_{2}} \rightarrow \mathcal{G}_{2}$ on the second factor is a locally trivial fiber bundle with fiber Trans $G_{0}\left(A_{2}, A_{1}\right) / \mathcal{N}_{G_{0}}\left(A_{1}\right)$.

- The projection $p_{1}: \mathcal{R}_{\mathcal{G}_{1}, \mathcal{G}_{2}} \rightarrow \mathcal{G}_{1}$ on the first factor is a finite covering with fiber $\operatorname{Trans}_{G_{0}}\left(A_{2}, A_{1}\right) / \mathcal{N}_{G_{0}}\left(A_{2}\right)$.

Proof Identifying $\mathcal{G}_{1}$ and $\mathcal{G}_{2}$ with the $G_{0}$-orbits of $A_{1}$ and $A_{2}$, we obtain identifications

$$
\mathcal{G}_{1}=G_{0} / \mathcal{N}_{G_{0}}\left(A_{1}\right), \quad \mathcal{G}_{2}=G_{0} / \mathcal{N}_{G_{0}}\left(A_{2}\right) .
$$

Consider the standard right action of $\mathcal{N}_{G_{0}}\left(A_{1}\right) \times \mathcal{N}_{G_{0}}\left(A_{2}\right)$ on $G_{0} \times G_{0}$

$$
\begin{aligned}
\left(G_{0} \times G_{0}\right) \times\left(\mathcal{N}_{G_{0}}\left(A_{1}\right) \times \mathcal{N}_{G_{0}}\left(A_{2}\right)\right) & \rightarrow G_{0} \times G_{0}, \\
\left(\left(g_{1}, g_{2}\right),\left(h_{1}, h_{2}\right)\right) & \mapsto\left(g_{1} h_{1}, g_{2} h_{2}\right),
\end{aligned}
$$

and notice that the set

$$
\widetilde{\mathcal{R}}=\left\{\left(g_{1}, g_{2}\right) \in G_{0} \times G_{0} \mid g_{1} A_{1} g_{1}^{-1} \supset g_{2} A_{2} g_{2}^{-1}\right\}
$$

is $\mathcal{N}_{G_{0}}\left(A_{1}\right) \times \mathcal{N}_{G_{0}}\left(A_{2}\right)$-invariant. In fact we have that

$$
\mathcal{R}_{\mathcal{G}_{1}, \mathcal{G}_{2}}=\tilde{\mathcal{R}} /\left(\mathcal{N}_{G_{0}}\left(A_{1}\right) \times \mathcal{N}_{G_{0}}\left(A_{2}\right)\right) .
$$

The projection $\tilde{p}_{2}: \widetilde{\mathcal{R}} \rightarrow G_{0}, \tilde{p}_{2}\left(g_{1}, g_{2}\right)=g_{2}$ has fibers

$$
\tilde{p}_{2}^{-1}\left(g_{2}\right)=\left\{\left(g_{1}, g_{2}\right) \in G_{0} \times G_{0} \mid g_{1}^{-1} g_{2} \in \operatorname{Trans}_{G_{0}}\left(A_{2}, A_{1}\right)\right\} .
$$

It follows that $\tilde{p}_{2}: \widetilde{\mathcal{R}} \rightarrow G_{0}$ is a locally trivial fiber bundle over $G_{0}$, with fiber $\operatorname{Trans}_{G_{0}}\left(A_{2}, A_{1}\right)$. From here we obtain that the projection

$$
p_{2}: \mathcal{R}_{\mathcal{G}_{1}, \mathcal{G}_{2}} \rightarrow \mathcal{G}_{2}
$$

is also a locally trivial fiber bundle with fibers $\operatorname{Trans}_{G_{0}}\left(A_{2}, A_{1}\right) / \mathcal{N}_{G_{0}}\left(A_{1}\right)$.

The same reasoning yields that the projection

$$
p_{1}: \mathcal{R}_{\mathcal{G}_{1}, \mathcal{G}_{2}} \rightarrow \mathcal{G}_{1}
$$


is also a locally trivial fiber bundle with fibers $\operatorname{Trans}_{G_{0}}\left(A_{2}, A_{1}\right) / \mathcal{N}_{G_{0}}\left(A_{2}\right)$. It follows from Lemma 3.3 that the quotient $\operatorname{Trans}_{G_{0}}\left(A_{2}, A_{1}\right) / \mathcal{N}_{G_{0}}\left(A_{2}\right)$ is finite. As it is a locally trivial fiber bundle with discrete fibers, $p_{1}: \mathcal{R}_{\mathcal{G}_{1}, \mathcal{G}_{2}} \rightarrow \mathcal{G}_{1}$ is a cover, as claimed.

Recall that we are identifying $\mathcal{K}$ with a subset of $\mathcal{G}$. Given $\mathcal{G}_{1}, \mathcal{G}_{2} \in \pi_{0}(\mathcal{G})$ with $\mathcal{G}_{1} \geq \mathcal{G}_{2}$, let $\mathcal{K}_{1} \subset \mathcal{G}_{1}$ and $\mathcal{K}_{2} \subset \mathcal{G}_{2}$ be the corresponding connected components of $\mathcal{K}$ and set

$$
\mathcal{O}_{\mathcal{G}_{1}, \mathcal{G}_{2}}=\mathcal{R}_{\mathcal{G}_{1}, \mathcal{G}_{2}} \cap\left(\mathcal{K}_{1} \times \mathcal{K}_{2}\right)=\left\{\left(H_{1}, H_{2}\right) \in \mathcal{K}_{1} \times \mathcal{K}_{2} \mid H_{1} \supset H_{2}\right\} .
$$

The same argument used to prove Lemma 3.13 yields:

Lemma 3.14 Fixing $\left(H_{1}, H_{2}\right) \in \mathcal{O}_{\mathcal{G}_{1}, \mathcal{G}_{2}}$, the following holds:

- The projection $p_{2}: \mathcal{O}_{\mathcal{G}_{1}, \mathcal{G}_{2}} \rightarrow \mathcal{K}_{2}$ on the second factor is a locally trivial fiber bundle with fiber $\operatorname{Trans}_{K_{0}}\left(H_{2}, H_{1}\right) / \mathcal{N}_{K_{0}}\left(H_{1}\right)$.

- The projection $p_{1}: \mathcal{O}_{\mathcal{G}_{1}, \mathcal{G}_{2}} \rightarrow \mathcal{K}_{1}$ on the first factor is a finite covering with fiber $\operatorname{Trans}_{K_{0}}\left(H_{2}, H_{1}\right) / \mathcal{N}_{K_{0}}\left(H_{2}\right)$.

\subsection{The real case}

Suppose now that the reductive group $G$ is defined over $\mathbb{R}$, let $G(\mathbb{R})$ be the group of real points and $K(\mathbb{R})$ a maximal compact subgroup of $G(\mathbb{R})$. Denote by $G(\mathbb{R})_{0}$ and $K(\mathbb{R})_{0}$, as always, the identity components of $G(\mathbb{R})$ and $K(\mathbb{R})$.

We will say that $A \subset G(\mathbb{R})$ is diagonalizable if its Zariski closure $\operatorname{Zar}(A) \subset G$ is diagonalizable and satisfies $A=\operatorname{Zar}(A)(\mathbb{R})$. A diagonalizable subgroup $A$ of $G(\mathbb{R})$ (resp. $K(\mathbb{R})$ ) is well-centered in $G(\mathbb{R})$ (resp. in $K(\mathbb{R})$ ) if it is equal to the center of its centralizer in $G(\mathbb{R})$ (resp. in $K(\mathbb{R})$ ). As above, we denote by $\mathcal{G}$ and $\mathcal{K}$ the set of all well-centered subgroups of $G(\mathbb{R})$ and $K(\mathbb{R})$, respectively.

We state now a few facts whose proofs we leave to the reader:

(1) If $A \subset G(\mathbb{R})$ is an abelian subgroup consisting of semisimple elements, then $\operatorname{Zar}(A)(\mathbb{R})$ (resp. $C\left(\mathcal{Z}_{G(\mathbb{R})}(A)\right)$ is the smallest diagonalizable subgroup of $G(\mathbb{R})$ (resp. smallest well-centered group in $G(\mathbb{R})$ ) containing $A$.

(2) If $A \subset G(\mathbb{R})$ is diagonalizable, then the intersection of $A$ and the unique maximal compact subgroup of $\operatorname{Zar}(A)$ is the unique maximal compact subgroup of $A$. Moreover, the Zariski closures of $A$ and its unique maximal compact subgroup agree. 
(3) If $A, A^{\prime} \subset G(\mathbb{R})$ are diagonalizable, then $\operatorname{Trans}_{G(\mathbb{R})}\left(A, A^{\prime}\right)$ is the subset of $\operatorname{Trans}_{G}\left(\operatorname{Zar}(A), \operatorname{Zar}\left(A^{\prime}\right)\right)$ fixed by complex conjugation and hence a real algebraic variety; as such, it has only finitely many connected components.

(4) If $A, A^{\prime} \subset G(\mathbb{R})$ are diagonalizable, $V \subset \operatorname{Trans}_{G(\mathbb{R})}\left(A, A^{\prime}\right)$ is a connected component, and $g_{0} \in V$, then the map $\mathcal{Z}_{G(\mathbb{R})}(A)_{0} \rightarrow V, h \mapsto g_{0} h$ is an isomorphism; here, $\mathcal{Z}_{G(\mathbb{R})}(A)_{0}$ is the connected component of the identity in the Lie group $\mathcal{Z}_{G(\mathbb{R})}(A)$.

(5) If $H_{1}, H_{2} \subset K(\mathbb{R})$ are subgroups, then the maps

$$
\begin{aligned}
\operatorname{Trans}_{K(\mathbb{R})}\left(H_{1}, H_{2}\right) \hookrightarrow \operatorname{Trans}_{G(\mathbb{R})}\left(H_{1}, H_{2}\right), \\
\operatorname{Trans}_{K(\mathbb{R})_{0}}\left(H_{1}, H_{2}\right) \hookrightarrow \operatorname{Trans}_{G(\mathbb{R})_{0}}\left(H_{1}, H_{2}\right),
\end{aligned}
$$

are homotopy equivalences.

(6) If $H \in \mathcal{K}$ is well-centered in $K(\mathbb{R})$, then $H$ is the unique maximal compact subgroup of the well-centered group $C\left(\mathcal{Z}_{G(\mathbb{R})}\right)(H) \in \mathcal{G}$.

(7) The actions $G(\mathbb{R}) \curvearrowright \mathcal{G}$ and $K(\mathbb{R}) \curvearrowright \mathcal{K}$ have finitely many orbits.

Remarks about the proofs of (1)-(7) Claims (1), (2) and (3) are just elementary considerations; compare with the proof of Lemma 2.14 for (3). Lemma 3.3 and (3) imply (4). The group $G(\mathbb{R})$ has a Cartan decomposition, now called polar decomposition; this said, Lemma 3.5 holds and hence the proofs of Lemma 3.4 and Lemma 3.7 transfer word-for-word to prove (5) and (6). Claim (7) follows from Lemma 3.9 and Lemma 2.14.

Before going any further, observe that (4) implies:

Lemma 3.3 (Real case) The Weyl groups $W_{A}=\mathcal{N}_{G(\mathbb{R})}(A) / \mathcal{Z}_{G(\mathbb{R})}(A)$ and $W_{A}^{0}=$ $\mathcal{N}_{G(\mathbb{R})_{0}}(A) / \mathcal{Z}_{G(\mathbb{R})_{0}}(A)$ are finite for every diagonalizable subgroup $A$ of $G(\mathbb{R})$.

Endow $\mathcal{G}\left(\right.$ resp. $\mathcal{K}$ ) with the manifold structure induced by the action of $G(\mathbb{R})_{0}$ (resp. $\left.K(\mathbb{R})_{0}\right)$. With facts (1)-(6) on hand, we can repeat word-for-word the proof of Proposition 3.11 and show:

Proposition 3.11 (Real case) The map $\iota: \mathcal{K} \rightarrow \mathcal{G}, \iota(H)=C\left(\mathcal{Z}_{G(\mathbb{R})}(H)\right)$ is an injective homotopy equivalence.

At this point we can define orderings on $\pi_{0}(\mathcal{G})$ and $\pi_{0}(\mathcal{K})$ exactly as we did in Section 3.5 in the complex case. We have then:

Lemma 3.12 (Real case) The homotopy equivalence $\iota: \mathcal{K} \rightarrow \mathcal{G}$ induces an orderpreserving bijection $\pi_{0}(\iota): \pi_{0}(\mathcal{K}) \rightarrow \pi_{0}(\mathcal{G})$. 
As for the complex case, we identify $\mathcal{K}$ and $\iota(\mathcal{K}) \subset \mathcal{G}$. For any two components $\mathcal{G}_{1}, \mathcal{G}_{2}$ of $\mathcal{G}$ with $\mathcal{G}_{1} \geq \mathcal{G}_{2}$ containing components $\mathcal{K}_{1} \subset \mathcal{G}_{1}$ and $\mathcal{K}_{2} \subset \mathcal{G}_{2}$ of $\mathcal{K}$ we define the sets $\mathcal{R}_{\mathcal{G}_{1}, \mathcal{G}_{2}}$ and $\mathcal{O}_{\mathcal{G}_{1}, \mathcal{G}_{2}}$ exactly as we did in (3.4) and (3.5). Armed with Facts (1)-(7) we can repeat word-for-word the same arguments as in Section 3.6 to prove:

Lemma 3.13 (Real case) Fixing $\left(A_{1}, A_{2}\right) \in \mathcal{R}_{\mathcal{G}_{1}, \mathcal{G}_{2}}$, the following hold:

- The projection $p_{2}: \mathcal{R}_{\mathcal{G}_{1}, \mathcal{G}_{2}} \rightarrow \mathcal{G}_{2}$ on the second factor is a locally trivial fiber bundle with fiber $\operatorname{Trans}_{G(\mathbb{R})_{0}}\left(A_{2}, A_{1}\right) / \mathcal{N}_{G(\mathbb{R})_{0}}\left(A_{1}\right)$.

- The projection $p_{1}: \mathcal{R}_{\mathcal{G}_{1}, \mathcal{G}_{2}} \rightarrow \mathcal{G}_{1}$ on the first factor is a finite covering with fiber $\operatorname{Trans}_{G(\mathbb{R})_{0}}\left(A_{2}, A_{1}\right) / \mathcal{N}_{G(\mathbb{R})_{0}}\left(A_{2}\right)$.

Lemma 3.14 (Real case) Fixing $\left(H_{1}, H_{2}\right) \in \mathcal{O}_{\mathcal{G}_{1}, \mathcal{G}_{2}}$, the following holds:

- The projection $p_{2}: \mathcal{O}_{\mathcal{G}_{1}, \mathcal{G}_{2}} \rightarrow \mathcal{K}_{2}$ on the second factor is a locally trivial fiber bundle with fiber $\operatorname{Trans}_{K(\mathbb{R})_{0}}\left(H_{2}, H_{1}\right) / \mathcal{N}_{K(\mathbb{R})_{0}}\left(H_{1}\right)$.

- The projection $p_{1}: \mathcal{O}_{\mathcal{G}_{1}, \mathcal{G}_{2}} \rightarrow \mathcal{K}_{1}$ on the first factor is a finite covering with fiber $\operatorname{Trans}_{K(\mathbb{R})_{0}}\left(H_{2}, H_{1}\right) / \mathcal{N}_{K(\mathbb{R})_{0}}\left(H_{2}\right)$.

\section{The canonical bundle over $\mathcal{G}$}

As before, let $G$ be the group of either complex or real points of a reductive algebraic group (defined over $\mathbb{R}$ in the latter case), let $K \subset G$ be a maximal compact subgroup, and recall that $\mathcal{G}$ is the manifold of all well-centered subgroups of $G$. As always $G_{0}$ and $K_{0}$ are the identity components of $G$ and $K$. In this section we study a certain flat bundle

$$
\pi: \mathcal{D} \rightarrow \mathcal{G}
$$

We take a flat bundle to mean a bundle endowed with a complete flat connection; in more concrete terms, the total space $\mathcal{D}$ is foliated in such a way that the restriction of $\pi$ to each leaf of the foliation is a covering of $\mathcal{G}$.

We also construct for all $k$ a map

$$
\Psi: \mathcal{D}(k) \rightarrow \operatorname{Hom}\left(\mathbb{Z}^{k}, G^{s}\right),
$$

where $\mathcal{D}(k) \rightarrow \mathcal{G}$ is the $k$-fold direct product of the bundle $\mathcal{D} \rightarrow \mathcal{G}$ with itself. Finally, we describe how $\Psi$ is related to the ordering of $\pi_{0}(\mathcal{G})$ and the isotopies induced by the flat connection. 


\subsection{Canonical bundle}

Endow $\mathcal{D}=\{(A, a) \in \mathcal{G} \times G \mid a \in A\}$ with the subspace topology and consider the projection

$$
\pi: \mathcal{D} \rightarrow \mathcal{G}, \quad(A, a) \mapsto A
$$

onto the first factor. We claim that $\pi: \mathcal{D} \rightarrow \mathcal{G}$ is a locally trivial bundle.

Fix a connected component $\mathcal{G}_{0}$ of $\mathcal{G}$ set

$$
\mathcal{D}_{\mathcal{G}_{0}}=\pi^{-1}\left(\mathcal{G}_{0}\right)=\left\{(A, a) \in \mathcal{G}_{0} \times G \mid a \in A\right\}
$$

and consider the restriction of $\pi$ to $\mathcal{D}_{\mathcal{G}_{0}}$. In order to prove that $\pi: \mathcal{D} \rightarrow \mathcal{G}$ is a locally trivial bundle, it suffices to prove it for $\pi: \mathcal{D}_{\mathcal{G}_{0}} \rightarrow \mathcal{G}_{0}$.

Fixing a base point $A \in \mathcal{G}_{0}$ recall that, by construction, $\mathcal{G}_{0}$ is equal to the orbit of $A$ under the action of $G_{0}$ by conjugation. It follows that the map

$$
\sigma_{0}: G_{0} \times A \rightarrow \mathcal{D}_{\mathcal{G}_{0}}, \quad(g, a) \mapsto\left(g A g^{-1}, g_{a g}{ }^{-1}\right)
$$

is surjective. The normalizer $\mathcal{N}_{G_{0}}(A)$ of $A$ in $G_{0}$ acts from the right on $G_{0} \times A$ as follows:

$$
\left(G_{0} \times A\right) \times \mathcal{N}_{G_{0}}(A) \rightarrow G_{0} \times A, \quad((g, a), h) \mapsto(g, a) \cdot h=\left(g h, h^{-1} a h\right) .
$$

A simple computation shows that the fibers of the map $\sigma_{0}$ agree with the $\mathcal{N}_{G_{0}}(A)$-orbits on $G_{0} \times A$. It follows that $\sigma_{0}$ descends to a homeomorphism

$$
\tau_{0}:\left(G_{0} \times A\right) / \mathcal{N}_{G_{0}}(A) \rightarrow \mathcal{D}_{\mathcal{G}_{0}} .
$$

Notice that, as $G_{0}$ acts transitively on $\mathcal{G}_{0}$, the base point $A$ yields a canonical identification $\mathcal{G}_{0}=G_{0} / \mathcal{N}_{G_{0}}(A)$. The projection $G_{0} \times A \rightarrow G_{0}$ onto the first factor induces a projection

$$
\pi^{\prime}:\left(G_{0} \times A\right) / \mathcal{N}_{G_{0}}(A) \rightarrow G_{0} / \mathcal{N}_{G_{0}}(A)=\mathcal{G}_{0} .
$$

Moreover, the projection $\pi^{\prime}$ and the homeomorphism $\tau_{0}$ are such that the following diagram commutes:

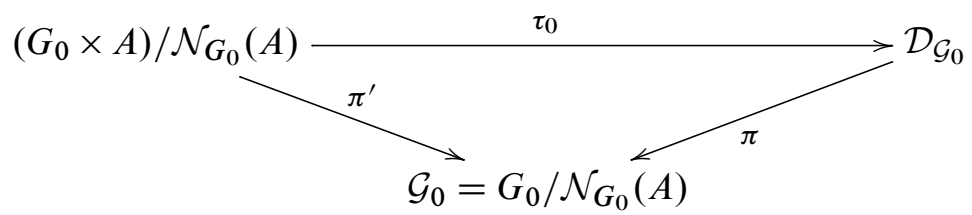

In particular, in order to prove that $\pi: \mathcal{D}_{\mathcal{G}_{0}} \rightarrow \mathcal{G}_{0}$ is a locally trivial bundle it will be sufficient to prove it for

$$
\pi^{\prime}:\left(G_{0} \times A\right) / \mathcal{N}_{G_{0}}(A) \rightarrow G_{0} / \mathcal{N}_{G_{0}}(A) .
$$


Recall that by Lemma 3.3, the group

$$
W_{A}^{0}=\mathcal{N}_{G_{0}}(A) / \mathcal{Z}_{G_{0}}(A)
$$

is finite. Taking into account that

$$
\begin{aligned}
\left(G_{0} \times A\right) / \mathcal{N}_{G_{0}}(A) & =\left(\left(G_{0} / \mathcal{Z}_{G_{0}}(A)\right) \times A\right) / W_{A}^{0}, \\
G_{0} / \mathcal{N}_{G_{0}}(A) & =\left(G_{0} / \mathcal{Z}_{G_{0}}(A)\right) / W_{A}^{0},
\end{aligned}
$$

we obtain a commuting diagram as follows:

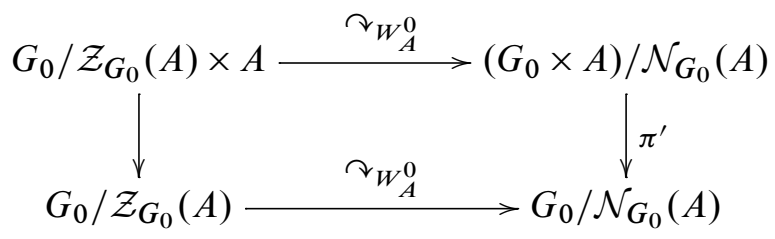

Here the left arrow is the projection onto the first factor, and the horizontal arrows are the orbit maps of the action of $W_{A}^{0}$.

By construction, $W_{A}^{0}$ acts on the trivial bundle $G_{0} / \mathcal{Z}_{G_{0}}(A) \times A \rightarrow G_{0} / \mathcal{Z}_{G_{0}}(A)$ by bundle homomorphisms. Moreover, the action is free and a fortiori discrete because $W_{A}^{0}$ is finite. It follows that the quotient $\pi^{\prime}:\left(G_{0} \times A\right) / \mathcal{N}_{G_{0}}(A) \rightarrow G_{0} / \mathcal{N}_{G_{0}}(A)$ is a locally trivial bundle. We have proved that

$$
\pi: \mathcal{D} \rightarrow \mathcal{G}
$$

is a locally trivial bundle. We will refer to this bundle as the canonical bundle over $\mathcal{G}$. Continuing with the same notation, endow now the trivial bundle $\left(G_{0} / \mathcal{Z}_{G_{0}}(A)\right) \times A \rightarrow$ $G_{0} / \mathcal{Z}_{G_{0}}(A)$ with the flat connection induced by the product structure. In other words, the leaves of the foliation associated to this flat connection are the sets of the form $\left(G_{0} / \mathcal{Z}_{G_{0}}(A)\right) \times\{a\}$. Since the group $W_{A}^{0}$ preserves the product structure, it follows that this flat connection descends to a flat connection on the canonical bundle. We have hence:

Proposition 4.1 The canonical bundle $\pi: \mathcal{D} \rightarrow \mathcal{G}$ is a flat locally trivial bundle.

\subsection{Horizontal lifts}

The use of having a flat connection on the bundle $\pi: \mathcal{D} \rightarrow \mathcal{G}$ is that to every curve $\gamma:[0,1] \rightarrow \mathcal{G}$ and to every point $a \in \pi^{-1}(\gamma(0)) \subset \mathcal{D}$ we can associate the horizontal lift of $\gamma$ starting at $a$. This is namely the unique curve

$$
\tilde{\gamma}:[0,1] \rightarrow \mathcal{D}
$$


with $\tilde{\gamma}(0)=a$ and $\pi(\tilde{\gamma}(t))=\gamma(t)$ for all $t \in[0,1]$, and whose image is contained in a single leaf of the foliation associated to the flat connection.

Our next goal is to give a concrete description of the horizontal lifts of curves $\gamma:[0,1] \rightarrow$ $\mathcal{G}$. To do so, let $\mathcal{G}_{0}$ be the connected component of $\mathcal{G}$ containing the image of $\gamma$, fix $A \in \mathcal{G}_{0}$, and recall that the map

$$
G_{0} \rightarrow \mathcal{G}_{0}, \quad g \mapsto g A g^{-1}
$$

is a fiber bundle with fiber $\mathcal{N}_{G_{0}}(A)$. Hence, every curve $\gamma:[0,1] \rightarrow \mathcal{G}$ admits a (far from unique) lift $[0,1] \rightarrow G_{0}, t \mapsto g_{t}$. The condition that $t \mapsto g_{t}$ is a lift of $\gamma$ amounts to

$$
\gamma(t)=g_{t} A g_{t}^{-1}=\left(g_{t} g_{0}^{-1}\right) \gamma(0)\left(g_{t} g_{0}^{-1}\right)^{-1} \text { for all } t .
$$

Notice that any element in $\pi^{-1}(\gamma(0)) \in \mathcal{D}$ is of the form $(\gamma(0), a)$, where $a \in \gamma(0)=$ $g_{0} A g_{0}^{-1} \subset G$. The curve

$$
\tilde{\gamma}:[0,1] \rightarrow \mathcal{D}, \quad t \mapsto\left(\left(g_{t} g_{0}^{-1}\right) \gamma(0)\left(g_{t} g_{0}^{-1}\right)^{-1},\left(g_{t} g_{0}^{-1}\right) a\left(g_{t} g_{0}^{-1}\right)^{-1}\right)
$$

is both parallel and a lift of $\gamma$; in other words, $\tilde{\gamma}$ is the horizontal lift of $\gamma$ with $\tilde{\gamma}(0)=(\gamma(0), a)$. We have proved:

Lemma 4.2 (Horizontal lift) Let $\gamma:[0,1] \rightarrow \mathcal{G}$ be a continuous curve and fix a point $A$ in the connected component of $\mathcal{G}$ containing the image of $\gamma$.

For any $a \in \gamma(0) \in \mathcal{G}$, the horizontal lift $\tilde{\gamma}:[0,1] \rightarrow \mathcal{D}$ of $\gamma$ starting at $\tilde{\gamma}(0)=(\gamma(0), a)$ is given by

$$
\tilde{\gamma}(t)=\left(\left(g_{t} g_{0}^{-1}\right) \gamma(0)\left(g_{t} g_{0}^{-1}\right)^{-1},\left(g_{t} g_{0}^{-1}\right) a\left(g_{t} g_{0}^{-1}\right)^{-1}\right),
$$

where $[0,1] \rightarrow G_{0}, t \mapsto g_{t}$ is an arbitrary curve with $\gamma(t)=g_{t} A g_{t}^{-1}$ for all $t$.

\subsection{The evaluation map}

Fix now $k$, recall that $\operatorname{Hom}\left(\mathbb{Z}^{k}, G^{s}\right)$ is the set of commuting semisimple $k$-tuples in $G$, and denote by

$$
\pi: \mathcal{D}(k) \rightarrow \mathcal{G}
$$

the $k^{\text {th }}$ fibered product of the bundle $\mathcal{D} \rightarrow \mathcal{G}$. In more concrete terms, the total space $\mathcal{D}(k)$ is given by

$$
\mathcal{D}(k)=\left\{\left(A, a_{1}, \ldots, a_{k}\right) \in \mathcal{G} \times G \times \cdots \times G \mid a_{1}, \ldots, a_{k} \in A\right\} .
$$

As a fibered product of flat bundles, $\mathcal{D}(k)$ is naturally endowed with a flat connection. 
Forgetting for a moment the bundle structure of $\mathcal{D}(k) \rightarrow \mathcal{G}$, we consider the following continuous map defined on the total space:

$$
\Psi: \mathcal{D}(k) \rightarrow \operatorname{Hom}\left(\mathbb{Z}^{k}, G^{s}\right), \quad\left(A, a_{1}, \ldots, a_{k}\right) \mapsto\left(a_{1}, \ldots, a_{k}\right) .
$$

Here we are identifying representations of $\mathbb{Z}^{k}$ with $k$-commuting tuples in $G$. Notice that the evaluation map $\Psi$ is well-defined because every well-centered group $A \in \mathcal{G}$ is, by definition, abelian and consists of semisimple elements.

Lemma 4.3 The evaluation map $\Psi: \mathcal{D}(k) \rightarrow \operatorname{Hom}\left(\mathbb{Z}^{k}, G^{s}\right)$ is surjective.

Proof Suppose that we are given $\rho \in \operatorname{Hom}\left(\mathbb{Z}^{k}, G^{s}\right)$. By Lemma 2.5, the abelian group $\rho\left(\mathbb{Z}^{k}\right)$ consists of semisimple elements. In particular, its Zariski closure is diagonalizable. It follows hence from Lemma 3.6 that $A=C\left(\mathcal{Z}_{G}\left(\rho\left(\mathbb{Z}^{k}\right)\right)\right)$ is a wellcentered group containing $\rho\left(\mathbb{Z}^{n}\right)$. Let $a_{i}=\rho\left(e_{i}\right)$ where $e_{1}, \ldots, e_{k}$ is the standard basis of $\mathbb{Z}^{k}$. Then $\left(A, a_{1}, \ldots, a_{k}\right) \in \mathcal{D}(k)$ and $\rho=\Psi\left(A, a_{1}, \ldots, a_{k}\right)$ by construction.

Before moving on we observe the following useful fact:

Lemma 4.4 We have $\Psi\left(g A g^{-1}, g \alpha g^{-1}\right)=g \Psi(A, \alpha) g^{-1}$ for all $(A, \alpha) \in \mathcal{D}(k)$ and $g \in G$.

Example: horizontal lifting and evaluation map for $\mathrm{SL}_{\boldsymbol{n}} \mathbb{C}$ In the case of $\mathrm{SL}_{n} \mathbb{C}$, the combination of horizontal lifting and the evaluation map just means the following: Suppose that a matrix $A \in \mathrm{SL}_{\mathbb{C}}$ is diagonalized by a direct sum decomposition $\mathbb{C}^{n}=$ $V_{1} \oplus \cdots \oplus V_{r}$ and let $\lambda_{i}$ be the homothety factor of $A$ when restricted to $V_{i}$. If we perturb each one of the spaces $V_{i}$ slightly to get some other space $V_{i}^{\prime}$, then there is a unique matrix $A^{\prime} \in \mathrm{SL}_{n} \mathbb{C}$, which, restricted to $V_{i}^{\prime}$, is a homothety with factor $\lambda_{i}$.

\subsection{Compatibility of horizontal lifts}

Suppose that we are given $\mathcal{G}_{1}, \mathcal{G}_{2} \in \pi_{0}(\mathcal{G})$ with $\mathcal{G}_{1} \geq \mathcal{G}_{2}$, and recall that this means that some, and hence every, $A_{2} \in \mathcal{G}_{2}$ can be conjugated into some, and hence into every, $A_{1} \in \mathcal{G}_{1}$. Recall that by Lemma 3.13, the projections of the set

$$
\mathcal{R}_{\mathcal{G}_{1}, \mathcal{G}_{2}}=\left\{\left(A_{1}, A_{2}\right) \in \mathcal{G}_{1} \times \mathcal{G}_{2} \mid A_{1} \supset A_{2}\right\}
$$

onto $\mathcal{G}_{1}$ and $\mathcal{G}_{2}$ are respectively a finite cover and a locally trivial fibration. 
Lemma 4.5 (Compatibility of horizontal lifts) Suppose that we are given a curve

$$
[0,1] \rightarrow \mathcal{R}_{\mathcal{G}_{1}, \mathcal{G}_{2}}, \quad t \mapsto\left(\gamma_{1}(t), \gamma_{2}(t)\right)
$$

and two points $\left(\gamma_{1}(0), \alpha_{1}\right) \in \mathcal{D}_{\mathcal{G}_{1}}(k)$ and $\left(\gamma_{2}(0), \alpha_{2}\right) \in \mathcal{D}_{\mathcal{G}_{2}}(k)$, and consider the horizontal lifts

$$
\tilde{\gamma}_{1}:[0,1] \rightarrow \mathcal{D}_{\mathcal{G}_{1}}(k), \quad \tilde{\gamma}_{2}:[0,1] \rightarrow \mathcal{D}_{\mathcal{G}_{2}}(k)
$$

of $\gamma_{1}$ and $\gamma_{2}$, with $\tilde{\gamma}_{i}(0)=\left(\gamma_{i}(0), \alpha_{i}\right)$ for $i=1,2$.

If $\Psi\left(\gamma_{1}(0), \alpha_{1}\right)=\Psi\left(\gamma_{2}(0), \alpha_{2}\right)$, then $\Psi\left(\tilde{\gamma}_{1}(t)\right)=\Psi\left(\tilde{\gamma}_{2}(t)\right)$ for all $t$.

Proof Setting $\left(A_{1}, A_{2}\right)=\left(\gamma_{1}(0), \gamma_{2}(0)\right)$, we obtain, as so often above, the identifications

$$
\mathcal{G}_{1}=G_{0} / \mathcal{N}_{G_{0}}\left(A_{1}\right), \quad \mathcal{G}_{2}=G_{0} / \mathcal{N}_{G_{0}}\left(A_{2}\right)
$$

Consider $G_{0} \rightarrow \mathcal{G}_{1}$ as a fiber bundle, and let $t \rightarrow g_{t}$ be any continuous lift of the curve $t \mapsto \gamma_{1}(t)$ with $g_{1}(0)=$ Id. Recall that by Lemma 3.13, the projection

$$
p_{1}: \mathcal{R}_{\mathcal{G}_{1}, \mathcal{G}_{2}} \rightarrow \mathcal{G}_{1}
$$

is a finite covering. The two curves

$$
\begin{aligned}
{[0,1] \rightarrow \mathcal{R}_{\mathcal{G}_{1}, \mathcal{G}_{2}}, \quad t \mapsto\left(\gamma_{1}(t), \gamma_{2}(t)\right), } \\
{[0,1] \rightarrow \mathcal{R}_{\mathcal{G}_{1}, \mathcal{G}_{2},}, \quad t \mapsto\left(g_{t} A_{1} g_{t}^{-1}, g_{t} A_{2} g_{t}^{-1}\right)=\left(\gamma_{1}(t), g_{t} A_{2} g_{t}^{-1}\right), }
\end{aligned}
$$

are lifts of $t \mapsto \gamma_{1}(t)$ under the covering $p_{1}: \mathcal{R}_{\mathcal{G}_{1}, \mathcal{G}_{2}} \rightarrow \mathcal{G}_{1}$; since both curves agree for $t=0$, it follows that $\gamma_{2}(t)=g_{t} A_{2} g_{t}^{-1}$ for all $t$. This proves that the curve $t \mapsto g_{t}$ is also a lift of the curve $t \mapsto \gamma_{2}(t)$ for the fiber bundle $G_{0} \rightarrow \mathcal{G}_{2}$.

Lemma 4.2 shows that the horizontal lift $\tilde{\gamma}_{i}:[0,1] \rightarrow \mathcal{D}_{i}$ of $\gamma_{i}$ with $\tilde{\gamma}_{i}(0)=\left(\gamma_{i}(0), \alpha_{i}\right)$ is given by

$$
\tilde{\gamma}_{i}(t)=\left(g_{t} \gamma_{i}(0) g_{t}^{-1}, g_{t} \alpha_{i} g_{t}^{-1}\right)
$$

for $i=1,2$. Lemma 4.4 implies that

$$
\Psi\left(\tilde{\gamma}_{i}(t)\right)=g_{t} \Psi\left(\tilde{\gamma}_{i}(0)\right) g_{t}^{-1} .
$$

The claim follows. 


\subsection{An interesting subbundle}

Continuing with the same notation, recall that by Lemma 3.1, every well-centered group $A \in \mathcal{G}$ contains a uniquely determined maximal compact subgroup $\bar{A}$. Consider the subset

$$
\overline{\mathcal{D}}=\{(A, a) \in \mathcal{G} \times G \mid a \in \bar{A}\} \subset \mathcal{D}
$$

and notice that the uniqueness of the maximal compact subgroups implies that $\overline{g A g^{-1}}=$ $g \bar{A} g^{-1}$ for all $A \in \mathcal{G}$ and all $g \in G$. It follows hence from Lemma 4.2 that for any $(A, a) \in \overline{\mathcal{D}}$ and any curve $\gamma:[0,1] \rightarrow \mathcal{G}$ with $\gamma(0)=A$, we have $\tilde{\gamma}(t) \in \overline{\mathcal{D}}$ for all $t$, where $\tilde{\gamma}$ is the horizontal lift of $\gamma$ with $\tilde{\gamma}(0)=(A, a)$. In other words, the subset $\overline{\mathcal{D}}$ of the total space of the bundle $\mathcal{D} \rightarrow \mathcal{G}$ is parallel.

This implies directly that the subset

$$
\overline{\mathcal{D}}(k)=\left\{\left(A, a_{1}, \ldots, a_{k}\right) \in \mathcal{G} \times G \times \cdots \times G \mid a_{1}, \ldots, a_{k} \in \bar{A}\right\}
$$

is a parallel subset of the bundle $\pi: \mathcal{D}(k) \rightarrow \mathcal{G}$. Observe now that any parallel subset of a flat bundle is a flat bundle in its own right. We have proved:

Lemma 4.6 $\pi: \overline{\mathcal{D}}(k) \rightarrow \mathcal{G}$ is a flat subbundle of $\pi: \mathcal{D}(k) \rightarrow \mathcal{G}$.

Recall at this point that $\mathcal{K}$ is the manifold of well-centered subgroups of $K$, and that by Proposition 3.11 the map

$$
\iota: \mathcal{K} \rightarrow \mathcal{G}, \quad \iota(H)=C\left(\mathcal{Z}_{G}(H)\right)
$$

is an injective homotopy equivalence. Recall also the definition (2.3) of $\operatorname{Hom}\left(\mathbb{Z}^{k}, K^{G}\right)$.

Lemma 4.7 With the same notation as above, we have:

(1) $\Psi(\overline{\mathcal{D}}(k))=\operatorname{Hom}\left(\mathbb{Z}^{k}, K^{G}\right)$.

(2) $\Psi\left(\overline{\mathcal{D}}(k) \cap \pi^{-1} \mathcal{K}\right)=\operatorname{Hom}\left(\mathbb{Z}^{k}, K\right)$.

Proof Recall that $\left(A, a_{1}, \ldots, a_{k}\right) \in \overline{\mathcal{D}}(k)$ if and only if $a_{1}, \ldots, a_{k} \in \bar{A}$ where $\bar{A}$ is the unique maximal compact subgroup of $A$. Since $\bar{A}$ is compact, there is $g \in G$ with $\bar{A} \subset g K g^{-1}$. In particular, $a_{1}, \ldots, a_{k} \in K^{G}=\bigcup_{g \in G} g K g^{-1}$; this proves that

$$
\Psi\left(A, a_{1}, \ldots, a_{k}\right)=\left(a_{1}, \ldots, a_{k}\right) \in \operatorname{Hom}\left(\mathbb{Z}^{k}, K^{G}\right)
$$

and hence that $\Psi(\mathcal{D}(k)) \subset \operatorname{Hom}\left(\mathbb{Z}^{k}, K^{G}\right)$. The opposite inclusion follows from a similar, simpler, argument (compare with Lemma 3.2); this proves (1). 
Continuing with the same notation assume now that $A \in \mathcal{K} \subset \mathcal{G}$ and recall that this means that there is a well-centered $H \subset K$ with $A=C\left(\mathcal{Z}_{G}(H)\right)$. Lemma 3.7 shows that $H$ is the maximal compact subgroup of $A$, ie, $H=\bar{A}$. Since $H \subset K$, it follows that $\Psi\left(A, a_{1}, \ldots, a_{k}\right)=\left(a_{1}, \ldots, a_{k}\right) \in \operatorname{Hom}\left(\mathbb{Z}^{k}, K\right)$ for any $a_{1}, \ldots, a_{k} \in \bar{A}=H$. This proves that $\Psi\left(\overline{\mathcal{D}}(k) \cap \pi^{-1} \mathcal{K}\right) \subset \operatorname{Hom}\left(\mathbb{Z}^{k}, K\right)$. The opposite inclusion follows again by a similar, again simpler, argument. This proves (2).

\section{The homotopic framework}

In this section we introduce some terminology and state a topological result, Proposition 5.3, which will play a key role in the proof of Theorem 1.1 . We defer its proof to the Appendix.

All topological spaces in this section will be CW-complexes, or more precisely, homeomorphic to CW-complexes.

\subsection{Regular coverings of simplicial complexes}

Suppose that $X$ is a (finite-dimensional, locally finite) simplicial complex and denote by $C(X)$ the set of all closed cells of $X$. Abusing terminology, we will not distinguish between the abstract complex $X$ and its geometric realization. We denote by $X^{(d)}$ the $d$-skeleton of $X$, ie, the simplicial complex consisting of all the cells of $X$ of dimension at most $d$; the set of closed $d$-dimensional cells we denote by $C_{d}(X)$. Recall also that an open covering of a space is good if all the possible intersections are either empty or contractible.

Definition An open covering $\mathcal{U}=\left\{U_{c}\right\}_{c \in C(X)}$ of a simplicial complex $X$ with components indexed by the set of closed cells of $X$ is regular if the following are satisfied:

- $\mathcal{U}$ is a good covering.

- For all $d, \bigcup_{c \in C_{d}(X)} \bar{U}_{c}$ is a closed regular neighborhood in $X \backslash X^{(d-1)}$ of $X^{(d)} \backslash \bigcup_{c \in C\left(X^{(d-1)}\right)} U_{c}$. Here $\bar{U}_{c}$ is the closure of $U_{c}$ in $X$.

- If $c, c^{\prime} \in C(X)$ are cells with $U_{c} \cap U_{c^{\prime}} \neq \varnothing$ then either $c \subset c^{\prime}$ or $c^{\prime} \subset c$. In particular, if $c$ and $c^{\prime}$ are distinct cells of the same dimension then $U_{c} \cap U_{c^{\prime}}=\varnothing$.

- For any cell $c \in C(X)$ we have $c \subset \cup_{c^{\prime} \subset c} U_{c^{\prime}}$.

The reader can find in Figure 1 an example of a regular covering of the 2-dimensional simplex. Clearly, every simplicial complex has a regular covering. Later we will need the following lemma, whose proof we leave to the reader: 


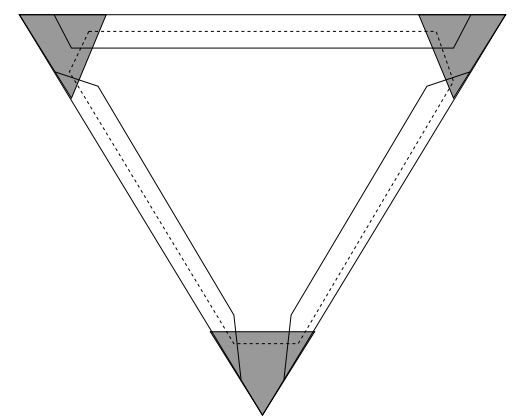

Figure 1: The shaded regions are the sets $U_{c}$ for the vertices. The solid lines bound the neighborhoods $U_{c}$ for the edges and the dashed line is the boundary of $U_{c}$ for the 2-dimensional cell.

Lemma 5.1 (Smashing Lemma) Let $X$ be simplicial complex and $\mathcal{U}=\left\{U_{c}\right\}_{c \in C(X)}$ a regular covering. There is a continuous (non-simplicial) map

$$
\Phi: X \rightarrow X
$$

with $\Phi\left(U_{c}\right)=\Phi(c)=c$ for any closed cell $c \in C(X)$.

In the particular case represented in Figure 1, the map $\Phi$ provided by Lemma 5.1 sends the shaded regions to the vertices, the regions bounded by solid lines to the edges, and the region bounded by the dashed line onto the whole simplex.

\subsection{Fibered relations}

Recall that a fibration is a continuous surjective map $\pi: S \rightarrow S^{\prime}$ with the homotopy lifting property. In this paper we are not lucky enough to find ourselves working with fibrations. What we encounter are fibered relations.

Definition A subset $\mathcal{R} \subset S \times S^{\prime}$ of the cartesian product $S \times S^{\prime}$ of two CW-complexes is a fibered relation if $\mathcal{R} \rightarrow S^{\prime}$ is a fibration.

If $K \subset S$ and $K^{\prime} \subset S^{\prime}$ are such that $\mathcal{O}=\mathcal{R} \cap\left(K \times K^{\prime}\right)$ is a fibered relation in $K \times K^{\prime}$, then we say that $(\mathcal{R}, \mathcal{O})$ is a fibered relation pair.

Remark In all cases of interest in this paper, the spaces $S, S^{\prime}, K, K^{\prime}, \mathcal{R}$ and $\mathcal{O}$ are smooth manifolds, and the projections are locally trivial fiber bundles.

It is easy to construct examples of fibered relations. For instance, $\mathcal{R}=S \times S^{\prime}$ is a fibered relation. The graph $\{(s, \pi(s)) \mid s \in S\} \subset S \times S^{\prime}$ of any fibration $\pi: S \rightarrow S^{\prime}$ is 
also a fibered relation. Finally, if $\widetilde{\mathcal{R}} \subset \widetilde{S} \times \widetilde{S}^{\prime}$ is a fibered relation and $\alpha: \widetilde{S} \rightarrow S$ and $\beta: \widetilde{S}^{\prime} \rightarrow S^{\prime}$ are coverings, then $\mathcal{R}=(\alpha \times \beta)(\widetilde{\mathcal{R}}) \subset S \times S^{\prime}$ is a fibered relation.

We will work simultaneously with various fibered relations. Suppose that we are given the following data:

(*) Let $\mathcal{P}=(\mathcal{P}, \geq)$ be a partially ordered set, $\mathcal{S}=\left\{S_{\Delta} \mid \Delta \in \mathcal{P}\right\}$ and $\mathcal{K}=\left\{K_{\Delta} \mid\right.$ $\Delta \in \mathcal{P}\}$ set of CW-complexes indexed by $\mathcal{P}$ with $K_{\Delta} \subset S_{\Delta}$ for all $\Delta$. For all $\Delta, \Delta^{\prime} \in \mathcal{P}$ with $\Delta \geq \Delta^{\prime}$ let

$$
\mathcal{R}_{\Delta, \Delta^{\prime}} \subset S_{\Delta} \times S_{\Delta^{\prime}} \text { and } \quad \mathcal{O}_{\Delta, \Delta^{\prime}}=\mathcal{R}_{\Delta, \Delta^{\prime}} \cap\left(K_{\Delta} \times K_{\Delta^{\prime}}\right) \subset K_{\Delta} \times K_{\Delta^{\prime}}
$$

such that $\left(\mathcal{R}_{\Delta, \Delta^{\prime}}, \mathcal{O}_{\Delta, \Delta^{\prime}}\right)$ is fibered relation pair. Set also

$$
\begin{aligned}
& \mathcal{R}=\left\{\mathcal{R}_{\Delta, \Delta^{\prime}} \mid \Delta \geq \Delta^{\prime}, \Delta, \Delta^{\prime} \in \mathcal{P}\right\}, \\
& \mathcal{O}=\left\{\mathcal{O}_{\Delta, \Delta^{\prime}} \mid \Delta \geq \Delta^{\prime}, \Delta, \Delta^{\prime} \in \mathcal{P}\right\} .
\end{aligned}
$$

Assuming $(*)$, let $X$ be a simplicial complex and recall that the set of all closed cells is denoted by $C(X)$. A $\mathcal{P}$-labeling of a simplicial complex $X$ is a map

$$
\delta: C(X) \rightarrow(\mathcal{P}, \geq)
$$

satisfying $\delta(c) \geq \delta\left(c^{\prime}\right)$ for all faces $c, c^{\prime} \in C(X)$ with $c \supset c^{\prime}$. A $\mathcal{P}$-labeled simplicial complex is a pair $(X, \delta)$ where $\delta$ is a $\mathcal{P}$-labeling of $X$.

Continuing with notation as in $(*)$, suppose that $(X, \delta)$ is a $\mathcal{P}$-labeled simplicial complex. To every regular cover $\mathcal{U}=\left\{U_{c}\right\}_{c \in C(X)}$ of $X$ we associate the bundle

$$
\mathcal{S} \times_{\delta} \mathcal{U}:=\bigsqcup_{c \in C(X)}\left(S_{\delta(c)} \times U_{c}\right) \rightarrow \bigsqcup_{c \in C(X)} U_{c},
$$

where $\bigsqcup$ stands for disjoint union. As a bundle $\mathcal{S} \times_{\delta} \mathcal{U}$ has no interest: it is just the disjoint union of trivial bundles. However, a rather non-trivial structure is added if we take into account the intersections of the components of $\mathcal{U}$ and that we have the set $\mathcal{R}$ of fibered relations.

Definition A section of $\left(\mathcal{S} \times{ }_{\delta} \mathcal{U}, \mathcal{R}\right)$ is a collection of continuous maps

$$
\left\{\sigma_{c}\right\}_{c \in C(X)}=\left\{\sigma_{c}: U_{c} \rightarrow S_{\delta(c)} \mid c \in C(X)\right\}
$$

with $\left(\sigma_{c}(x), \sigma_{c^{\prime}}(x)\right) \in \mathcal{R}_{\delta(c), \delta\left(c^{\prime}\right)}$ for all $c, c^{\prime} \in C(X), c \supset c^{\prime}$, and all $x \in U_{c} \cap U_{c^{\prime}}$.

Two sections $\left\{\sigma_{c}\right\}_{c \in C(X)}$ and $\left\{\sigma_{c}^{\prime}\right\}_{c \in C(X)}$ of $\left(\mathcal{S} \times_{\delta} \mathcal{U}, \mathcal{R}\right)$ are homotopic if for every $c \in C(X)$ there is a continuous map

$$
[0,1] \times U_{c} \rightarrow S_{\delta(c)}, \quad(t, x) \mapsto \sigma_{c}^{t}(x)
$$


with $\sigma_{c}^{0}=\sigma_{c}$ and $\sigma_{c}^{1}=\sigma_{c}^{\prime}$, such that $\left\{\sigma_{c}^{t}\right\}_{c \in C(X)}$ is a section for all $t$. If $W \subset X$ is such that $\sigma_{c}^{t}(x)=\sigma_{c}(x)$ for all $c \in C(X)$, all $x \in W \cap U_{c}$, and all $t$, then we say that the homotopy $\left\{\sigma_{c}^{t}\right\}_{c \in C(X)}$ is constant on $W$.

Notation In order to lighten the burden of notation we will from now on write

$$
\mathcal{S} \times{ }_{\delta} \mathcal{U}=\left(\mathcal{S} \times{ }_{\delta} \mathcal{U}, \mathcal{R}\right)
$$

dropping any reference to the set $\mathcal{R}$ of fibered relations.

\subsection{Fibered relations associated to a group $G$}

Let $G$ be the group of either complex or real points of a reductive algebraic group (defined over $\mathbb{R}$ in the latter case) and $K \subset G$ a maximal compact subgroup. As in Section 3.4 let $\mathcal{G}$ be the manifold of all well-centered subgroups of $G$ and consider $\pi_{0}(\mathcal{G})$ with the partial order introduced in Section 3.5. Recall that every component $\mathcal{G}_{i}$ contains a unique component $\mathcal{K}_{i}$ of the manifold $\mathcal{K}$ of well-centered groups in $K$. The following is a direct consequence of Lemmas 3.13 and 3.14:

Lemma 5.2 Let $\mathcal{G}_{1}, \mathcal{G}_{2}$ and $\mathcal{K}_{1}, \mathcal{K}_{2}$ be connected components of the manifolds $\mathcal{G}$ and $\mathcal{K}$ of well-centered groups in $G$ and $K$, respectively; assume that $\mathcal{G}_{1} \geq \mathcal{G}_{2}$ and that $\mathcal{K}_{i} \subset \mathcal{G}_{i}$ for $i=1,2$. The pair

$$
\left(\mathcal{R}_{\mathcal{G}_{1}, \mathcal{G}_{2}}, \mathcal{O}_{\mathcal{G}_{1}, \mathcal{G}_{2}}\right) \subset\left(\mathcal{G}_{1} \times \mathcal{G}_{2}, \mathcal{K}_{1} \times \mathcal{K}_{2}\right),
$$

where $\mathcal{R}_{\mathcal{G}_{1}, \mathcal{G}_{2}}$ and $\mathcal{O}_{\mathcal{G}_{1}, \mathcal{G}_{2}}$ are as in (3.4) and (3.5), is a fibered relation pair.

To conclude the analogy with $(*)$, set

$$
\begin{gathered}
\mathcal{R}_{G}=\left\{\mathcal{R}_{\mathcal{G}_{1}, \mathcal{G}_{2}} \mid \mathcal{G}_{1} \geq \mathcal{G}_{2}, \mathcal{G}_{1}, \mathcal{G}_{2} \in \pi_{0}(\mathcal{G})\right\}, \\
\mathcal{O}_{G}=\left\{\mathcal{O}_{\mathcal{G}_{1}, \mathcal{G}_{2}} \mid \mathcal{G}_{1} \geq \mathcal{G}_{2}, \mathcal{G}_{1}, \mathcal{G}_{2} \in \pi_{0}(\mathcal{G})\right\} .
\end{gathered}
$$

Given now a $\pi_{0}(\mathcal{G})$-labeled simplicial complex $(X, \delta)$ and a regular cover $\mathcal{U}$ of $X$ we consider the bundle $\mathcal{G} \times{ }_{\delta} \mathcal{U}$ and sections therein as defined above.

Remark To clarify the nature of the different objects, when working with $\mathcal{G} \times{ }_{\delta} \mathcal{U}$ we will denote by $\mathcal{G}_{\delta(c)}$ the connected component $\delta(c) \in \pi_{0}(\mathcal{G})$ for $c \in C(X)$.

The following result asserts that, up to slightly reducing the domain, every section of $\mathcal{G} \times{ }_{\delta} \mathcal{U}$ can be homotoped to a section of $\mathcal{K} \times{ }_{\delta} \mathcal{U}$. 
Proposition 5.3 Let $G$ be the group of either complex or real points of a reductive algebraic group, $K \subset G$ a maximal compact subgroup, $\mathcal{G}$ and $\mathcal{K}$ the manifolds of well-centered groups in $G$ and $K$, and consider the sets of fibered relations $\mathcal{R}_{G}$ and $\mathcal{O}_{G}$ defined in (5.1) and (5.2).

Let $(X, \delta)$ be a $\pi_{0}(\mathcal{G})$-labeled complex, and $\mathcal{U}=\left\{U_{c}\right\}_{c \in C(X)}$ and $\mathcal{V}=\left\{V_{c}\right\}_{c \in C(X)}$ regular covers of $X$ with $V_{c}$ relatively compact in $U_{c}$ for all $c \in C(X)$. Finally, let $\left\{\sigma_{c}\right\}_{c \in C(X)}$ be a section of $\mathcal{G} \times_{\delta} \mathcal{U}$, and $W \subset X$ with $\sigma_{c}(w) \in \mathcal{K}_{\delta(c)}$ for all $c \in C(X)$ and all $w \in U_{c} \cap W$.

The restriction of $\left\{\sigma_{c}\right\}_{c \in C(X)}$ to $\mathcal{G} \times_{\delta} \mathcal{V}$ is homotopic to a section $\left\{\sigma_{c}^{\prime}\right\}_{c \in C(X)}$ with $\sigma_{c}^{\prime}\left(V_{c}\right) \subset \mathcal{K}_{\delta(c)}$ for all $c \in C(X)$; moreover the homotopy is constant on $W$.

Recall that by Proposition 3.11 the manifold $\mathcal{G}$ retracts to the submanifold $\mathcal{K}$. In light of this fact, the reader may be willing to believe that the proof of Proposition 5.3 is a very tedious and painful exercise in topology; we thereby defer it to the Appendix. Essentially the idea is to observe that the set of fibered relation pairs $\left(\mathcal{R}_{G}, \mathcal{O}_{G}\right)$ satisfies some conditions (transitivity, reflexivity, simultaneous lifting and relative retraction properties) that allow us to work with sets of fibered relations very much as one would with single fibrations.

\section{Proof of Theorem 1.1}

In this section we prove Theorem 1.1 assuming the following result:

Proposition 6.1 Let $G$ be the group of complex points of a connected reductive algebraic group, $K$ a maximal compact subgroup of $G$, and recall that $G$ acts on $\operatorname{Hom}\left(\mathbb{Z}^{k}, G\right)$ by conjugation. There is a continuous $G$-equivariant map

$$
\Phi:[0,1] \times\left(\operatorname{Hom}\left(\mathbb{Z}^{k}, G\right), \operatorname{Hom}\left(\mathbb{Z}^{k}, K\right)\right) \rightarrow\left(\operatorname{Hom}\left(\mathbb{Z}^{k}, G\right), \operatorname{Hom}\left(\mathbb{Z}^{k}, K\right)\right)
$$

with the following properties:

- $\Phi(0, \rho)=\rho$ and $\Phi(1, \rho) \in \operatorname{Hom}\left(\mathbb{Z}^{k}, K^{G}\right)$ for all $\rho \in \operatorname{Hom}\left(\mathbb{Z}^{k}, G\right)$.

- There is an open covering $\mathcal{W}$ of $\operatorname{Hom}\left(\mathbb{Z}^{k}, K^{G}\right)$ such that for every $W \in \mathcal{W}$ one of the following non-exclusive alternatives holds:

(1) All the elements in $\Phi(\{1\} \times W)$ have the same orbit type.

(2) All the elements in $W$ have the same orbit type.

If moreover $G$ is defined over $\mathbb{R}$, then $\Phi$ is equivariant under complex conjugation. 
Recall that by Lemma 3.2, $\operatorname{Hom}\left(\mathbb{Z}^{k}, K^{G}\right)$ is the set of representations whose image is contained in a conjugate of $K$. In particular, $\operatorname{Hom}\left(\mathbb{Z}^{k}, K^{G}\right)$ is contained in the set $\operatorname{Hom}\left(\mathbb{Z}^{k}, G^{s}\right)$ of semi-simple representations and contains $\operatorname{Hom}\left(\mathbb{Z}^{k}, K\right)$. Recall also that two elements $\rho, \rho^{\prime}$ in $\operatorname{Hom}\left(\mathbb{Z}^{k}, G\right)$ have the same orbit type if their stabilizers $\operatorname{Stab}_{G}(\rho)$ and $\operatorname{Stab}_{G}\left(\rho^{\prime}\right)$ are conjugate in $G$.

We will prove Proposition 6.1 in Section 8.

Theorem 1.1 Let $G$ be the group of complex or real points of a reductive algebraic group, defined over $\mathbb{R}$ in the latter case, and $K \subset G$ a maximal compact subgroup. For all $k$ there is a weak retraction of $\operatorname{Hom}\left(\mathbb{Z}^{k}, G\right)$ to $\operatorname{Hom}\left(\mathbb{Z}^{k}, K\right)$.

For the sake of concreteness, suppose that $G$ is the group of complex points. With the same notation as in Proposition 6.1, let

$$
\phi:\left(\operatorname{Hom}\left(\mathbb{Z}^{k}, K^{G}\right), \operatorname{Hom}\left(\mathbb{Z}^{k}, K\right)\right) \rightarrow\left(\operatorname{Hom}\left(\mathbb{Z}^{k}, K^{G}\right), \operatorname{Hom}\left(\mathbb{Z}^{k}, K\right)\right)
$$

be the restriction of $\Phi(1, \cdot)$ to $\operatorname{Hom}\left(\mathbb{Z}^{k}, K^{G}\right)$ and notice that $G$-equivariance implies that

$$
\operatorname{Stab}_{G}(\phi(\rho)) \supset \operatorname{Stab}_{G}(\rho)
$$

for all $\rho \in \operatorname{Hom}\left(\mathbb{Z}^{k}, G\right)$.

Theorem 1.1 will follow once we show that $\phi$ is homotopic, as a map of pairs, to a map whose image is contained in $\operatorname{Hom}\left(\mathbb{Z}^{k}, K\right)$. To see why this is the case suppose that

$$
[0,1] \times \operatorname{Hom}\left(\mathbb{Z}^{k}, K^{G}\right) \rightarrow \operatorname{Hom}\left(\mathbb{Z}^{k}, K^{G}\right), \quad(t, \rho) \mapsto \phi^{t}(\rho)
$$

is a homotopy with $\phi^{0}=\phi$, with $\phi^{1}\left(\operatorname{Hom}\left(\mathbb{Z}^{k}, K^{G}\right)\right) \subset \operatorname{Hom}\left(\mathbb{Z}^{k}, K\right)$, and with $\phi^{t}\left(\operatorname{Hom}\left(\mathbb{Z}^{k}, K\right)\right) \subset \operatorname{Hom}\left(\mathbb{Z}^{k}, K\right)$ for all $t$. Then the map

$$
f:[0,1] \times \operatorname{Hom}\left(\mathbb{Z}^{k}, G\right) \rightarrow \operatorname{Hom}\left(\mathbb{Z}^{k}, G\right)
$$

given by

$$
\begin{array}{ll}
f(t, \rho)=\Phi(3 t, \rho) & \text { for } t \in\left[0, \frac{1}{3}\right], \\
f(t, \rho)=\Phi(3 t-1, \phi(\rho)) & \text { for } t \in\left[\frac{1}{3}, \frac{2}{3}\right], \\
f(t, \rho)=\phi^{3 t-2}(\phi(\rho)) & \text { for } t \in\left[\frac{2}{3}, 1\right],
\end{array}
$$

is a continuous homotopy between $f(0, \rho)=\rho$ and $f(1, \rho)=\phi^{1}(\phi(\rho))$. Clearly, the image of $f(1, \cdot)$ is contained in $\operatorname{Hom}\left(\mathbb{Z}^{k}, K\right)$ because the image of $\phi^{1}(\cdot)$ is. Also, since $\Phi(t, \cdot)$ and $\phi^{t}(\cdot)$ preserve $\operatorname{Hom}\left(\mathbb{Z}^{k}, K\right)$ for all $t$ it follows that the same is true for $f(t, \cdot)$. In other words, $f(\cdot, \cdot)$ is a weak retraction of $\operatorname{Hom}\left(\mathbb{Z}^{k}, G\right)$ to $\operatorname{Hom}\left(\mathbb{Z}^{k}, K\right)$. 
It remains to prove that the homotopy $\phi^{t}$ exists. We sketch briefly how we will prove its existence, but before doing so refer to Section 3.4, Section 4.3 and Section 4.5 for the definitions of $\mathcal{G}, \mathcal{K}, \pi: \mathcal{D}(k) \rightarrow \mathcal{G}$ and $\pi: \overline{\mathcal{D}}(k) \rightarrow \mathcal{G}$.

The strategy Considering $X=\operatorname{Hom}\left(\mathbb{Z}^{k}, K^{G}\right)$ as a simplicial complex with set of cells $C(X)$, we will associate to the map $\phi$

- a regular covering $\mathcal{U}=\left\{U_{c}\right\}_{c \in C(X)}$ of $X$, and

- a labeling $\delta: C(X) \rightarrow \pi_{0}(\mathcal{G})$.

In order to remind the reader of the nature of the different objects we will denote by $\mathcal{G}_{\delta(c)}$ the connected component $\delta(c)$ of $\mathcal{G}$; accordingly, we also set $\overline{\mathcal{D}}_{\delta(c)}(k)=\pi^{-1}\left(\mathcal{G}_{\delta(c)}\right)$. Besides the covering $\mathcal{U}$ and the labeling $\delta$, we also associate to the map $\phi$

- a section $\bigsqcup_{c \in C(X)} \sigma_{c}: \bigsqcup_{c \in C(X)} U_{c} \rightarrow \bigsqcup_{c \in C(X)} \mathcal{G}_{\delta(c)}$ of $\mathcal{G} \times_{\delta} \mathcal{U}$, and

- a map $\bigsqcup_{c \in C(X)} \tilde{\sigma}_{c}: \bigsqcup_{c \in C(X)} U_{c} \rightarrow \bigsqcup_{c \in C(X)} \overline{\mathcal{D}}_{\delta(c)}(k)$

such that the following diagram commutes:

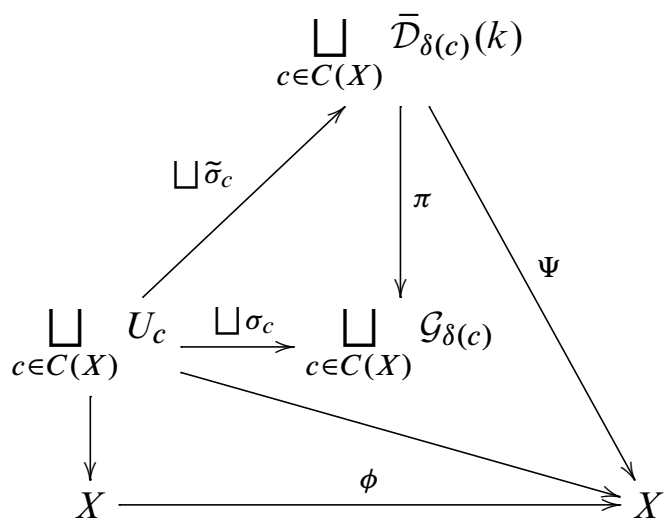

Here $\Psi: \overline{\mathcal{D}}(k) \rightarrow \operatorname{Hom}\left(\mathbb{Z}^{k}, G\right)$ is the evaluation map defined in Section 4.3 and the unlabeled vertical arrow is the obvious map $\bigsqcup U_{c} \rightarrow \bigcup U_{c}=X$.

These objects in place, we apply Proposition 5.3 to obtain a homotopy of the section $\sqcup \sigma_{c}$ to a section which takes values in $\mathcal{K} \times{ }_{\delta} \mathcal{U}$. The flatness of the bundle $\pi: \overline{\mathcal{D}} \rightarrow \mathcal{G}$ allows us to lift this homotopy to a homotopy of the map $\bigsqcup \widetilde{\sigma}_{c}$. Composing the elements of this last homotopy with the evaluation map $\Psi$, we obtain a one parameter family of maps $\bigsqcup U_{c} \rightarrow X$ with the property that the image of the final map is contained in $\operatorname{Hom}\left(\mathbb{Z}^{k}, K\right)$. The compatibility of horizontal lifts, Lemma 4.5, implies then that this one parameter family of maps descends to a one parameter family of maps $X \rightarrow X$ 
starting with $\phi$ and ending with a map whose image is $\operatorname{Hom}\left(\mathbb{Z}^{k}, K\right)$; this is the desired homotopy.

Still assuming that $G$ is the group of complex points, we now put the proof of Theorem 1.1 into full swing:

The simplicial complex Consider the triangulation $\mathcal{T}_{0}$ of $\operatorname{Hom}\left(\mathbb{Z}^{k}, K^{G}\right)$ provided by Proposition 2.8 and notice that by Proposition 6.1 there is a refinement $\mathcal{T}$ of $\mathcal{T}_{0}$ with the property that every closed cell $c \in \mathcal{T}$ has an open neighborhood $W_{c}$ such that:

(1) either any two $\rho, \rho^{\prime} \in \phi\left(W_{c}\right)$ have the same orbit type, or

(2) any two $\rho, \rho^{\prime} \in W_{c}$ have the same orbit type.

Consider from now on

$$
X=\left(\operatorname{Hom}\left(\mathbb{Z}^{k}, K^{G}\right), \mathcal{T}\right)
$$

as a simplicial complex and notice that by Proposition $2.8, \operatorname{Hom}\left(\mathbb{Z}^{k}, K\right) \subset X$ is a subcomplex.

Remark As a triangulation, $\mathcal{T}$ is locally finite. In particular, we can assume without loss of generality that $W_{c^{\prime}} \subset W_{c}$ for any two closed cells $c, c^{\prime}$ of $X$ with $c^{\prime} \subset c$.

The labeling With the same notation as in Section 3, let $\mathcal{G}$ and $\mathcal{K}$ be the manifolds of all well-centered groups in $G$ and $K$ and recall that by Proposition 3.11 the map

$$
\iota: \mathcal{K} \rightarrow \mathcal{G}, \quad H \mapsto C\left(\mathcal{Z}_{G}(H)\right)
$$

is an injective homotopy equivalence. Recall also that the inclusion relation between well-centered groups yields an order on the sets $\pi_{0}(\mathcal{G})$ and $\pi_{0}(\mathcal{K})$ of connected components of $\mathcal{G}$ and $\mathcal{K}$ and that the map $\iota$ induces an order-preserving bijection $\pi_{0}(\mathcal{G}) \rightarrow \pi_{0}(\mathcal{K})$ by Lemma 3.12 .

Our next goal is to define a $\pi_{0}(\mathcal{G})$-labeling on the complex $X$, but first we remind the reader of a few facts:

- $\operatorname{Stab}_{G}(\rho)=\mathcal{Z}_{G}\left(\rho\left(\mathbb{Z}^{k}\right)\right)$ for all $\rho \in \operatorname{Hom}\left(\mathbb{Z}^{k}, G\right)$.

- By Lemma 3.6, the group $C\left(\mathcal{Z}_{G}\left(\rho\left(\mathbb{Z}^{k}\right)\right)\right)=C\left(\operatorname{Stab}_{G}(\rho)\right)$ is well-centered, ie, an element of $\mathcal{G}$, for any $\rho \in \operatorname{Hom}\left(\mathbb{Z}^{k}, K^{G}\right)$.

- By Lemma 3.10, $\rho, \rho^{\prime} \in \operatorname{Hom}\left(\mathbb{Z}^{k}, K^{G}\right)$ have the same $G_{0}$-orbit type if and only if $C\left(\operatorname{Stab}_{G}(\rho)\right)$ and $C\left(\operatorname{Stab}_{G}\left(\rho^{\prime}\right)\right)$ belong to the same connected component of $\mathcal{G}$.

Notice that it follows from these observations, together with (1) and (2) above, that for every cell $c \in C(X)$ one of the following non-mutually exclusive alternatives holds: 
$\left(1^{\prime}\right)$ Either there is a component $\mathcal{G}_{0}$ of $\mathcal{G}$ with

$$
C\left(\operatorname{Stab}_{G}(\phi(\rho))\right), C\left(\operatorname{Stab}_{G}\left(\phi\left(\rho^{\prime}\right)\right)\right) \in \mathcal{G}_{0}
$$

for all $\rho, \rho^{\prime} \in W_{c}$, or

$\left(2^{\prime}\right)$ there is a component $\mathcal{G}_{1}$ of $\mathcal{G}$ with

$$
C\left(\operatorname{Stab}_{G}(\rho)\right), C\left(\operatorname{Stab}_{G}\left(\rho^{\prime}\right)\right) \in \mathcal{G}_{1}
$$

for all $\rho, \rho^{\prime} \in W_{c}$.

Keeping the notation above, we define a function $\delta: C(X) \rightarrow \pi_{0}(\mathcal{G})$ by

$$
\begin{array}{ll}
\delta(c)=\mathcal{G}_{0} & \text { if }\left(1^{\prime}\right) \text { is satisfied, and } \\
\delta(c)=\mathcal{G}_{1} & \text { otherwise. }
\end{array}
$$

We claim that $(X, \delta)$ is a labeled complex.

Lemma 6.2 The function $\delta: C(X) \rightarrow \pi_{0}(\mathcal{G})$ is a labeling, ie, $\delta(c) \geq \delta\left(c^{\prime}\right)$ for all $c, c^{\prime} \in C(X)$ with $c \supset c^{\prime}$.

Proof Suppose first that $\left(1^{\prime}\right)$ holds for $c$. The assumption that $W_{c^{\prime}} \subset W_{c}$ for all $c^{\prime} \subset c$ implies that $\left(1^{\prime}\right)$ also holds for $c^{\prime}$. This proves that $\delta\left(c^{\prime}\right)=\delta(c)$.

Assume now that $\left(1^{\prime}\right)$ is not satisfied for $c$. In particular

$$
C\left(\operatorname{Stab}_{G}(\rho)\right), C\left(\operatorname{Stab}_{G}\left(\rho^{\prime}\right)\right) \in \delta(c)
$$

for all $\rho, \rho^{\prime} \in W_{c}$. Notice that if $\left(1^{\prime}\right)$ is also not satisfied for $c^{\prime}$, then we again have $\delta\left(c^{\prime}\right)=\delta(c)$. So, suppose that $\left(1^{\prime}\right)$ is satisfied for $c^{\prime}$. Recall that for $\rho \in W_{c^{\prime}} \subset W_{c}$ we have

$$
\operatorname{Stab}_{G}(\rho) \subset \operatorname{Stab}_{G}(\phi(\rho))
$$

by Proposition 6.1. It follows hence from Lemma 3.6 that

$$
C\left(\operatorname{Stab}_{G}(\rho)\right) \supset C\left(\operatorname{Stab}_{G}(\phi(\rho))\right) .
$$

Since $C\left(\operatorname{Stab}_{G}(\rho)\right) \in \delta(c)$ and $C\left(\operatorname{Stab}_{G}(\phi(\rho))\right) \in \delta\left(c^{\prime}\right)$ this means that $\delta(c) \geq \delta\left(c^{\prime}\right)$, as we needed to prove.

From now on we consider $X=(X, \delta)$ as a $\pi_{0}(\mathcal{G})$-labeled complex. We will denote the connected component $\delta(c)$ of $\mathcal{G}$ by $\mathcal{G}_{\delta(c)}$ for $c \in C(X)$. 
The cover and the section Fix once and for all a regular cover $\mathcal{U}=\left\{U_{c}\right\}_{c \in C(X)}$ of $X$ with $U_{c} \subset W_{c}$ for all $c \in C(X)$. Our next goal is to associate to the map $\phi$ a section $\left\{\sigma_{c}\right\}_{c \in C(X)}$ of

$$
\mathcal{G} \times{ }_{\delta} \mathcal{U}=\bigsqcup_{c \in C(X)} \mathcal{G}_{\delta(c)} \times U_{c} \rightarrow \bigsqcup_{c \in C(X)} U_{c}
$$

For $\rho \in U_{c}$ we set, with the same notation as before,

$$
\begin{aligned}
& \sigma_{c}(\rho)=C\left(\operatorname{Stab}_{G}(\phi(\rho))\right) \text { if }\left(1^{\prime}\right) \text { is satisfied, and } \\
& \sigma_{c}(c)=C\left(\operatorname{Stab}_{G}(\rho)\right) \quad \text { otherwise, }
\end{aligned}
$$

and notice that by definition $\sigma_{c}(\rho) \in \mathcal{G}_{\delta(c)}$. We have hence for all $c \in C(X)$ a map

$$
\sigma_{c}: U_{c} \rightarrow \mathcal{G}_{\delta(c)} .
$$

We summarize in the following lemma a few properties of the maps $\sigma_{c}$ :

Lemma 6.3 Each one of the maps $\sigma_{c}: U_{c} \rightarrow \mathcal{G}_{\delta(c)}$ is continuous. Moreover, the collection of maps $\left\{\sigma_{c}\right\}_{c \in C(X)}$ is a section of $\mathcal{G} \times{ }_{\delta} \mathcal{U}$.

Proof Suppose that we have a sequence $\left(\rho_{n}\right)$ in $U_{c}$ converging to $\rho \in U_{c}$ and suppose for the sake of concreteness that $\left(1^{\prime}\right)$ holds for $c$. Observe that the continuity of $\phi$ implies that $\phi\left(\rho_{n}\right) \rightarrow \phi(\rho)$. Noticing that

$$
\phi(\rho) \in \operatorname{Hom}\left(\mathbb{Z}^{k}, K^{G}\right) \subset \operatorname{Hom}\left(\mathbb{Z}^{k}, G^{s}\right)
$$

is a semisimple representation, it follows from Theorem 2.7 that the orbit of $\phi(\rho)$ under $G$ is closed in the affine variety $\operatorname{Hom}\left(\mathbb{Z}^{k}, G\right)$. Hence, Theorem 2.4 and its following remark imply there is a sequence $\left(g_{n}\right)$ in $g$ converging to Id such that

$$
g_{n} \operatorname{Stab}_{G}\left(\phi\left(\rho_{n}\right)\right) g_{n}^{-1} \subset \operatorname{Stab}_{G}(\phi(\rho)) .
$$

On the other hand, since we are assuming that $\left(1^{\prime}\right)$ is satisfied, we know $\operatorname{Stab}_{G}(\phi(\rho))$ and $\operatorname{Stab}\left(\phi\left(\rho_{n}\right)\right)$ are conjugate for all $n$, hence

$$
g_{n} \operatorname{Stab}_{G}\left(\phi\left(\rho_{n}\right)\right) g_{n}^{-1}=\operatorname{Stab}_{G}(\phi(\rho)) .
$$

It follows now directly from $g_{n} \rightarrow \mathrm{Id}$ and the definition of $\sigma_{c}$ that

$$
\sigma_{c}\left(\rho_{n}\right) \rightarrow \sigma_{c}(\rho)
$$

as we needed to prove. This proves that the map $\sigma_{c}$ is continuous if $\left(1^{\prime}\right)$ is satisfied for $c$. If $\left(1^{\prime}\right)$ is not satisfied then the argument is word-for-word the same, once we remove the appearance of $\phi$ everywhere. 
It remains to prove that the collection of maps $\left\{\sigma_{c}\right\}_{c \in C(X)}$ is a section of $\mathcal{G} \times{ }_{\delta} \mathcal{U}$. The argument is the same as that we used in Lemma 6.2 to prove that $\delta$ is a labeling, and we leave it to the reader to make the necessary modifications.

Before moving on, notice that it follows directly from the definitions that

$$
\sigma_{c}\left(U_{c} \cap \operatorname{Hom}\left(\mathbb{Z}^{k}, K\right)\right) \subset \mathcal{K}_{\delta(c)}
$$

for all $c \in C(X)$.

The lift Consider as in Section 4 the $k^{\text {th }}$ power

$$
\pi: \mathcal{D}(k) \rightarrow \mathcal{G}
$$

of the canonical bundle and set $\mathcal{D}_{\delta(c)}(k)=\pi^{-1}\left(\mathcal{G}_{\delta(c)}\right)$ for $c \in C(X)$. In more concrete terms:

$$
\mathcal{D}_{\delta(c)}(k)=\left\{\left(A, a_{1}, \ldots, a_{k}\right) \in \mathcal{G}_{\delta(c)} \times G \times \cdots \times G \mid a_{i} \in A \forall i\right\}
$$

Denote also by $\overline{\mathcal{D}}_{\delta(c)}(k)$ the intersection of $\mathcal{D}_{\delta(c)}(k)$ with the total space of the flat subbundle $\pi: \overline{\mathcal{D}}(k) \rightarrow \mathcal{G}$ introduced in Section 4.5. Explicitly, $\overline{\mathcal{D}}_{\delta(c)}$ is the set of those $\left(A, a_{1}, \ldots, a_{k}\right) \in \mathcal{D}_{\delta(c)}$ such that the elements $a_{1}, \ldots, a_{k}$ belong to the unique maximal compact subgroup of $A$.

Notice now that for $c \in C(X)$ and $\rho \in U_{c}$ we have that

$$
(\phi(\rho))\left(\mathbb{Z}^{n}\right) \subset C\left(\operatorname{Stab}_{G}(\phi(\rho))\right) \subset C\left(\operatorname{Stab}_{G}(\rho)\right)
$$

and hence that $(\phi(\rho))\left(\mathbb{Z}^{n}\right) \subset \sigma_{c}(\rho)$. Also, as $\phi(\rho) \in \operatorname{Hom}\left(\mathbb{Z}^{k}, K^{G}\right)$ it follows that $(\phi(\rho))\left(e_{i}\right)$ is contained in the unique maximal compact subgroup of $\sigma_{c}(\rho)$, where $e_{1}, \ldots, e_{k}$ is the standard basis of $\mathbb{Z}^{k}$. It follows from these two considerations that for all $c \in C(X)$ the map

$$
\tilde{\sigma}_{c}: U_{c} \rightarrow \overline{\mathcal{D}}_{\delta(c)}(k), \quad \tilde{\sigma}_{c}(\rho)=\left(\sigma_{c}(\rho),(\phi(\rho))\left(e_{1}\right), \ldots,(\phi(\rho))\left(e_{k}\right)\right)
$$

is well-defined.

It follows directly from the construction and the definitions that

$$
\left(\pi \circ \tilde{\sigma}_{c}\right)(\rho)=\sigma_{c}(\rho) \text { and } \phi(\rho)=\left(\Psi \circ \tilde{\sigma}_{c}\right)(\rho)
$$

for all $\rho \in U_{c}$. Here, $\Psi: \mathcal{D}(k) \rightarrow \operatorname{Hom}\left(\mathbb{Z}^{k}, G\right)$ is the evaluation map defined in Section 4.3; recall that $\Psi(\overline{\mathcal{D}}(k)) \subset \operatorname{Hom}\left(\mathbb{Z}^{k}, K^{G}\right)$ by Lemma 4.7.

At this point we have constructed all the objects in the commuting diagram (6.2) above. It remains to construct the homotopy of $\phi$. 
The homotopy Proposition 5.3 asserts that, up to reducing each one of the open sets $U_{c}$ slightly, there is a continuous map

$$
\bigsqcup_{c \in C(X)}\left([0,1] \times U_{c}\right) \rightarrow \bigsqcup_{c \in C(X)} \mathcal{G}_{\delta(c)}
$$

sending $(t, \rho) \in[0,1] \times U_{c}$ to $\sigma_{c}^{t}(\rho)$ such that the following properties are satisfied:

(i) For all $t$ the collection of maps $\left\{\sigma_{c}^{t}\right\}_{c \in C(X)}$ is a section of $\mathcal{G} \times{ }_{\delta} \mathcal{U}$.

(ii) $\sigma_{c}^{0}=\sigma_{c}$ for all $c \in C(X)$.

(iii) $\sigma_{c}^{1}\left(U_{c}\right) \subset \mathcal{K}_{\delta(c)}$ for all $c \in C(X)$.

(iv) $\sigma_{c}^{t}(\rho)=\sigma_{c}(\rho)$ for all $c \in C(X)$ and all $\rho \in U_{c}$ with $\sigma_{c}(\rho) \in \mathcal{K}_{\delta(c)}$.

At this point, recall that for all $c \in C(X)$, the bundle $\pi: \overline{\mathcal{D}}_{\delta(c)} \rightarrow \mathcal{G}_{\delta(c)}$ is flat, and that we have the map $\widetilde{\sigma}_{c}: U_{c} \rightarrow \overline{\mathcal{D}}_{\delta(c)}$ with

$$
\pi \circ \tilde{\sigma}_{c}=\sigma_{c}=\sigma_{c}^{0} .
$$

Consider for all $c \in C(X)$ the horizontal lift

$$
[0,1] \times U_{c} \rightarrow \overline{\mathcal{D}}_{\delta(c)}, \quad(t, \rho) \mapsto \widetilde{\sigma}_{c}^{t}(\rho)
$$

of the homotopy $[0,1] \times U_{c} \rightarrow \mathcal{G}_{\delta(c)},(t, \rho) \mapsto \sigma_{c}^{t}(\rho)$ satisfying $\widetilde{\sigma}_{c}^{0}(\cdot)=\widetilde{\sigma}_{c}(\cdot)$. As the horizontal lift of a continuous homotopy, the map $(t, \rho) \mapsto \widetilde{\sigma}_{c}^{t}(\rho)$ is continuous as well.

Lemma 6.4 The map

$$
\bigsqcup_{c \in C(X)}\left([0,1] \times U_{c}\right) \rightarrow X, \quad[0,1] \times U_{c} \ni(t, \rho) \mapsto \Psi\left(\widetilde{\sigma}_{c}^{t}(\rho)\right)
$$

descends to a continuous map

$$
[0,1] \times X \rightarrow X, \quad(t, \rho) \mapsto \phi^{t}(\rho)
$$

with $\phi^{0}=\phi$. Moreover, $\phi^{t}(\rho)=\phi(\rho)$ for all $t$ and all $\rho \in \operatorname{Hom}\left(\mathbb{Z}^{k}, K\right)$.

Proof Suppose that we have two cells $c, c^{\prime}$ with $U_{c} \cap U_{c}^{\prime} \neq \varnothing$, and notice that, by the definition of a regular covering, one of them is contained in the other, say, $c^{\prime} \subset c$. This implies, hence, that $\delta(c) \geq \delta\left(c^{\prime}\right)$. The condition that the maps $\left\{\sigma_{c}^{t}\right\}_{c \in C(X)}$ form a section of $\mathcal{G} \times{ }_{\delta} \mathcal{U}$ implies that for all $\rho \in U_{c} \cap U_{c^{\prime}}$ we have

$$
\left(\sigma_{c}^{t}(\rho), \sigma_{c^{\prime}}^{t}(\rho)\right) \in \mathcal{R}_{\delta(c), \delta\left(c^{\prime}\right)}
$$

for all $t$. Recall now that we have

$$
\Phi\left(\widetilde{\sigma}_{c}(\rho)\right)=\phi(\rho)=\Phi\left(\widetilde{\sigma}_{c^{\prime}}(\rho)\right)
$$


and that $\tilde{\sigma}_{c}^{t}(\rho)$ and $\tilde{\sigma}_{c^{\prime}}^{t}(\rho)$ are both obtained through horizontal lifting. Lemma 4.5 asserts that we have

$$
\Phi\left(\widetilde{\sigma}_{c}^{t}(\rho)\right)=\Phi\left(\widetilde{\sigma}_{c^{\prime}}^{t}(\rho)\right)
$$

for all $t$. We have proved that the map

$$
\begin{aligned}
& \bigsqcup_{c \in C(X)}\left([0,1] \times U_{c}\right) \rightarrow X, \\
& {[0,1] \times U_{c} \ni(t, \rho) \mapsto \widetilde{\Phi}\left(\sigma_{c}^{t}(\rho)\right),}
\end{aligned}
$$

descends to a map

$$
[0,1] \times X \rightarrow X, \quad(t, \rho) \mapsto \phi^{t}(\rho)
$$

given by $\phi^{t}(\rho)=\Phi\left(\widetilde{\sigma}_{c}^{t}(\rho)\right)$ for any $c \in C(X)$ with $\rho \in U_{c}$; notice that by construction $\phi^{0}=\phi$. The map $(t, \rho) \mapsto \phi^{t}(\rho)$ is continuous because its restriction for each one of the sets $[0,1] \times U_{c}$ is the continuous map $(t, \rho) \mapsto \Phi\left(\widetilde{\sigma}_{c}^{t}(\rho)\right)$.

Finally, suppose that $\rho \in \operatorname{Hom}\left(\mathbb{Z}^{k}, K\right)$ and that $c \in C(X)$ is a cell with $\rho \in U_{c}$. By (6.3) we have $\sigma_{c}(\rho) \in \mathcal{K}_{\delta(c)}$ and hence, by (iv) above, we have that $\sigma_{c}^{t}(\rho)=\sigma_{c}(\rho)$ for all $t$. This in turn implies that $\widetilde{\sigma}_{c}^{t}(\rho)=\widetilde{\sigma}_{c}(\rho)$ for all $t$. Hence

$$
\phi^{t}(\rho)=\Phi\left(\widetilde{\sigma}_{c}^{t}(\rho)\right)=\Phi\left(\widetilde{\sigma}_{c}(\rho)\right)=\phi(\rho)
$$

again for all $t$. This concludes the proof of Lemma 6.4.

The end At this point, we are nearly done with the proof of Theorem 1.1. It only remains to prove that the final map $\phi^{1}$ of the homotopy provided by Lemma 6.4 takes values in $\operatorname{Hom}\left(\mathbb{Z}^{k}, K\right)$. In order to see that this is the case, recall that by construction we have $\sigma_{c}^{1}\left(U_{c}\right) \subset \mathcal{K}_{\delta(c)}$ for all $c \in C(X)$, hence

$$
\tilde{\sigma}_{c}^{1}\left(U_{c}\right) \subset \overline{\mathcal{D}}_{\delta(c)} \cap \pi^{-1}\left(\mathcal{K}_{\delta(c)}\right) .
$$

Lemma 4.7 implies now that

$$
\phi^{1}\left(U_{c}\right)=\Phi\left(\widetilde{\sigma}_{c}^{1}\left(U_{c}\right)\right) \subset \operatorname{Hom}\left(\mathbb{Z}^{k}, K\right) .
$$

The claim follows because the sets $U_{c}$ cover $\operatorname{Hom}\left(\mathbb{Z}^{k}, K^{G}\right)$.

We have proved that the map (6.1) is homotopic as a map of pairs to a map that takes values in $\operatorname{Hom}\left(\mathbb{Z}^{k}, K\right)$. This concludes the proof of Theorem 1.1 if $G$ is the group of complex points.

The real case Suppose now that $G=G(\mathbb{R})$ is the group of real points, and let $K(\mathbb{R})$ and $K$ be maximal compact subgroups of $G(\mathbb{R})$ and $G$ with $K(\mathbb{R}) \subset K$. The map 
$\Phi(1, \cdot)$ provided by Proposition 6.1 is equivariant under complex conjugation. In particular, $\Phi(1, \cdot)$ maps $\operatorname{Hom}\left(\mathbb{Z}^{k}, G(\mathbb{R})\right)$ to $\operatorname{Hom}\left(\mathbb{Z}^{k}, K(\mathbb{R})^{G(\mathbb{R})}\right)$, and $\operatorname{Hom}\left(\mathbb{Z}^{k}, K(\mathbb{R})\right)$ to itself. Consider the map

$$
\phi: \operatorname{Hom}\left(\mathbb{Z}^{k}, K(\mathbb{R})^{G(\mathbb{R})}\right) \rightarrow \operatorname{Hom}\left(\mathbb{Z}^{k}, K(\mathbb{R})^{G(\mathbb{R})}\right)
$$

obtained by restricting $\Phi(1, \cdot)$ to $\operatorname{Hom}\left(\mathbb{Z}^{k}, K(\mathbb{R})^{G(\mathbb{R})}\right)$. Now we can repeat word-forword, referring to the appropriate results in Section 2.7 and Section 3.7, the argument given in the complex case.

The next two sections are devoted to the proof of Proposition 6.1.

\section{Approximated Jordan decomposition}

The main tool needed to prove Proposition 6.1 is what we call the approximated Jordan decomposition. This construction is the goal of the present section.

\subsection{Jordan decomposition}

Recall that $g \in \mathrm{SL}_{n} \mathbb{C}$ is unipotent if one of the following three equivalent conditions are satisfied:

- $g$ has characteristic polynomial $\chi_{g}(T)=(T-1)^{n}$.

- $g-\mathrm{Id}$ is nilpotent, meaning that $(g-\mathrm{Id})^{n}=0$.

- $g$ - Id has spectral radius $\rho(g-$ Id $)=0$ where the spectral radius of a linear transformation is the maximum of the norms of its eigenvalues.

Given arbitrary $g \in \mathrm{SL}_{n} \mathbb{C}$, there is $h \in \mathrm{SL}_{n} \mathbb{C}$ such that $h g h^{-1}$ is in Jordan normal form. Denote by $a$ the diagonal part of $h g h^{-1}$, and set

$$
g_{s}=h^{-1} a h \quad \text { and } \quad g_{u}=g g_{s}^{-1} .
$$

By construction, $g_{s}$ is semisimple and $g_{u}$ is unipotent. Moreover, it is well-known that $g_{s}$ and $g_{u}$ are polynomials in $g$, meaning that $g_{s}, g_{u} \in \mathbb{C}[g] \subset M_{n} \mathbb{C}$. In particular, if $h \in \mathrm{SL}_{n} \mathbb{C}$ commutes with $g$, then it also commutes with $g_{s}$ and $g_{u}$. Suppose now that $G \subset \mathrm{SL}_{n} \mathbb{C}$ is an algebraic group. A priori, the semisimple part $g_{s}$ and the unipotent part $g_{u}$ of $g \in G$ only belong to the ambient group $\mathrm{SL}_{n} \mathbb{C}$, but in fact $g_{s}, g_{u} \in G$ as well [7]. To summarize, we have:

Jordan decomposition Let $G$ be an algebraic group. For every $g \in G$ there are uniquely determined elements, $g_{s} \in G$ semisimple and $g_{u} \in G$ unipotent, with $g=$ $g_{s} g_{u}$, and such that if $h \in G$ commutes with $g$, then it also commutes with each of $g_{s}$ and $g_{u}$. 
It is a rather unlucky fact that the Jordan decomposition $g \mapsto\left(g_{s}, g_{u}\right)$ is not continuous; the goal of this section is to construct a continuous approximation of this decomposition. Letting $G$ act on itself and on $G \times G$ by conjugation, we prove:

Theorem 1.7 Let $G \subset \mathrm{SL}_{n} \mathbb{C}$ be an algebraic group and $H_{1}, \ldots, H_{r} \subset G$ a finite collection of algebraic subgroups of $G$. For every $\epsilon>0$ there is a continuous $G-$ equivariant map

$$
G \rightarrow G \times G, \quad g \mapsto\left(g_{s}^{\epsilon}, g_{u}^{\epsilon}\right)
$$

satisfying the following properties for all $g \in G$ :

(1) $g=g_{s}^{\epsilon} g_{u}^{\epsilon}=g_{u}^{\epsilon} g_{s}^{\epsilon}$.

(2) $g_{s}^{\epsilon}$ and $g_{u}^{\epsilon}$ are polynomials in $g$, ie, they belong to $\mathbb{C}[g] \subset M_{n} \mathbb{C}$.

(3) If $h \in G$ commutes with $g \in G$, then $h$ also commutes with $g_{s}^{\epsilon}$ and $g_{u}^{\epsilon}$.

(4) $g_{s}^{\epsilon}$ is semisimple and $g_{u}^{\epsilon}-$ Id has spectral radius at most $\epsilon$.

(5) If $K \subset G$ is a maximal compact group then $g_{s}^{\epsilon}, g_{u}^{\epsilon} \in K$ for all $g \in K$.

(6) $g_{s}^{\epsilon}, g_{s}^{u} \in H_{i}$ for all $g \in H_{i}$.

(7) If moreover $G$ is defined over $\mathbb{R}$, then $\bar{g}_{s}^{\epsilon}=\overline{g_{s}^{\epsilon}}$ and $\bar{g}_{u}^{\epsilon}=\overline{g_{u}^{\epsilon}}$, where ${ }^{-}$is complex conjugation.

We refer to the decomposition

$$
g=g_{s}^{\epsilon} g_{u}^{\epsilon}
$$

provided by Theorem 1.7 as the approximated Jordan decomposition for the following reason. The elements $g_{s}^{\epsilon}$ and $g_{u}^{\epsilon}$ have the same commutativity properties as those of the Jordan decomposition, and while $g_{u}^{\epsilon}$ is not unipotent, it is almost so: recall that $u \in \mathrm{SL}_{n} \mathbb{C}$ is unipotent if and only if $\rho(u-\mathrm{Id})=0$.

To prove Theorem 1.7 we will work in the ambient group $\mathrm{SL}_{n} \mathbb{C}$ : we construct the desired map there and then restrict it to $G$. In this setting, it is easy to understand the reason for the lack of continuity of the Jordan decomposition and essentially the idea behind the construction of the proof of Theorem 1.7 is to preempt its discontinuities. The precise statement we will prove is the following:

Proposition 7.1 For every $\epsilon>0$ there is a continuous $\mathrm{SL}_{n} \mathbb{C}$-equivariant map

$$
\mathrm{SL}_{n} \mathbb{C} \rightarrow \mathrm{SL}_{n} \mathbb{C} \times \mathrm{SL}_{n} \mathbb{C}, \quad g \mapsto\left(g_{s}^{\epsilon}, g_{u}^{\epsilon}\right)
$$

satisfying the following properties for all $g \in \mathrm{SL}_{n} \mathbb{C}$ :

(1) $g=g_{s}^{\epsilon} g_{u}^{\epsilon}=g_{u}^{\epsilon} g_{s}^{\epsilon}$. 
(2) $g_{s}^{\epsilon}$ and $g_{u}^{\epsilon}$ are polynomials in $g$, ie, they belong to $\mathbb{C}[g] \subset M_{n} \mathbb{C}$.

(3) $g_{s}^{\epsilon}$ is semisimple and $g_{u}^{\epsilon}$ - Id has at most spectral radius $\epsilon$.

(4) $g_{s}^{\epsilon}, g_{u}^{\epsilon} \in \mathrm{SU}_{n}$ for all $g \in \mathrm{SU}_{n}$.

(5) $\bar{g}_{s}^{\epsilon}=\overline{g_{s}^{\epsilon}}$ and $\bar{g}_{u}^{\epsilon}=\overline{g_{u}^{\epsilon}}$, where $\bar{r}$ is complex conjugation.

Moreover, if $G_{1}, \ldots, G_{r} \subset \mathrm{SL}_{n} \mathbb{C}$ is a finite collection of algebraic groups then the map (7.1) can be chosen in such a way that $g_{s}^{\epsilon}, g_{u}^{\epsilon} \in G_{i}$ for all $g \in G_{i}$.

With the same notation as in Theorem 1.7, notice that we may conjugate $G \subset \mathrm{SL}_{n} \mathbb{C}$ so that the maximal compact subgroup $K$ is contained in $\mathrm{SU}_{n}$. In particular, Theorem 1.7 follows immediately once we have proved Proposition 7.1. Before proving the latter, we require a few preparatory remarks, but first we introduce some notation used throughout the remainder of this section.

We denote by $\mathbb{S}_{n} \subset \mathrm{SL}_{n} \mathbb{C}$ the standard maximal torus of $\mathrm{SL}_{n} \mathbb{C}$, ie, the group of diagonal matrices with determinant 1 . Recall that the Weyl-group

$$
W\left(\mathbb{S}_{n}, \mathrm{SL}_{n} \mathbb{C}\right)=\mathcal{N}_{\mathrm{SL}_{n} \mathbb{C}}\left(\mathbb{S}_{n}\right) / \mathcal{Z}_{\mathrm{SL}_{n} \mathbb{C}}\left(\mathbb{S}_{n}\right)
$$

is the symmetric group $\Sigma_{n}$ on $n$ symbols. Here, $\mathcal{N}_{\mathrm{SL}_{n} \mathbb{C}}\left(\mathbb{S}_{n}\right)$ and $\mathcal{Z}_{\mathrm{SL}_{n} \mathbb{C}}\left(\mathbb{S}_{n}\right)$ are, as always, the normalizer and the centralizer of $\mathbb{S}_{n}$ in $\mathrm{SL}_{n} \mathbb{C}$.

We identify $\mathbb{S}_{n}$ with a subset of $\mathbb{C}^{n}$ :

$$
\mathbb{S}_{n} \simeq\left\{\left(\lambda_{i}\right)_{i=1, \ldots, n} \in \mathbb{C}^{n} \mid \lambda_{1} \cdot \ldots \cdot \lambda_{n}=1\right\}, \quad\left(\begin{array}{lll}
\lambda_{1} & & \\
& \ddots & \\
& & \lambda_{n}
\end{array}\right) \simeq\left(\lambda_{1}, \ldots, \lambda_{n}\right)
$$

Under this identification, the action of the Weyl group $\Sigma_{n}$ on $\mathbb{S}_{n}$ is just the restriction of the standard action on $\mathbb{C}^{n}$.

\subsection{Diagonal matrices conjugated into an algebraic group}

It is well-known that if $G$ is a connected algebraic group then every semisimple element in $G$ can be conjugated into every maximal torus $\mathbb{T} \subset G$. We discuss now an extension of this observation for disconnected groups.

Lemma 7.2 Let $G \subset \mathrm{SL}_{n} \mathbb{C}$ be an algebraic group. There is a finite collection $\mathcal{T}$ of algebraic subgroups of the diagonal group $\mathbb{S}_{n} \subset \mathrm{SL}_{n} \mathbb{C}$ such that for every $g \in G$ and $h \in \mathrm{SL}_{n} \mathbb{C}$ with $h g h^{-1} \in \mathbb{S}_{n}$ there is $T \in \mathcal{T}$ such that $g \in h^{-1} T h \subset G$. 
Proof Let $R_{u}(G)$ be the unipotent radical of $G$ and observe that by the Levi decomposition theorem there is $G^{\prime} \subset G$ reductive with $G=R_{u}(G) \rtimes G^{\prime}$. Recall also that every semisimple element in $G$ can be conjugated into $G^{\prime}$. Since every element that can be conjugated into $\mathbb{S}_{n}$ is semisimple, it suffices to prove that the statement of the lemma holds for $G^{\prime}$. In other words, we may assume without loss of generality that $G$ is reductive to begin with.

By Theorem 2.1, the smooth variety $\mathrm{SL}_{n} \mathbb{C} / G$ is affine. Consider the left action

$$
\mathbb{S}_{n} \times \mathrm{SL}_{n} \mathbb{C} / G \rightarrow \mathrm{SL}_{n} \mathbb{C} / G, \quad(t, h G) \mapsto t h G
$$

and observe that it has a principal orbit by Theorem 2.4. In fact, since every subgroup of $\mathbb{S}_{n}$ is reductive, the argument in the proof of Proposition 2.6 shows that the action (7.3) has finitely many orbit types. Observe however that since $\mathbb{S}_{n}$ is abelian, finiteness of orbit types implies in fact that the collection $\mathcal{T}$ of stabilizers of points under the action (7.3) is finite.

Given now $g \in G$ and $h \in \mathrm{SL}_{n} \mathbb{C}$ suppose that $h g h^{-1}=t \in \mathbb{S}_{n}$. This means that $h^{-1} t h \in G$ and hence that $t h G=h G$, so $t \in \operatorname{Stab}_{\mathbb{S}_{n} \curvearrowright \mathrm{SL}_{n} \mathbb{C} / G}(h G)$. This proves that there is $T=\operatorname{Stab}_{\mathbb{S}_{n} \curvearrowright \mathrm{SL}_{n} \mathbb{C} / G}(h G) \in \mathcal{T}$ with $g \in h^{-1} T h$. Continuing with the same notation, observe that for every $t^{\prime} \in T$ we have $t^{\prime} h G=h G$ and hence $h^{-1} t^{\prime} h \in G$. This proves that $h^{-1} T h \subset G$, as claimed.

\subsection{Musings on sets of eigenvalues}

Suppose that we are given $g \in \mathrm{SL}_{n} \mathbb{C}$, and let $g_{s}$ be its semisimple part with respect to the Jordan decomposition. As $g_{s}$ is diagonalizable, there is $h \in \mathrm{SL}_{n} \mathbb{C}$ with $h g_{s} h^{-1} \in$ $\mathbb{S}_{n}$. Notice that if $h^{\prime} \in \mathrm{SL}_{n} \mathbb{C}$ is another element with $h^{\prime} g_{s} h^{-1} \in \mathbb{S}_{n}$, then $h g_{s} h^{-1}$ and $h^{\prime} g_{s} h^{\prime-1}$ differ by an element of the Weyl group $\Sigma_{n}$. Hence we can associate to $g$ the uniquely determined element $\mathcal{E}_{g} \in \mathbb{S}_{n} / \Sigma_{n}$.

Identifying $\mathbb{S}_{n}$ as above with a subset of $\mathbb{C}^{n}$, let $\left[\lambda_{1}, \ldots, \lambda_{n}\right] \in \mathbb{C}^{n} / \Sigma_{n}$ be an unordered $n$-tuple of complex numbers representing $\mathcal{E}_{g}$. It follows from the definition that $\mathcal{E}_{g}=$ $\left[\lambda_{1}, \ldots, \lambda_{n}\right]$ is the unordered tuple of the eigenvalues of $g$, counted with multiplicity. We can also consider the unordered tuple $\mathcal{E}_{g}=\left[\lambda_{1}, \ldots, \lambda_{n}\right]$ as an atomic measure on $\mathbb{C}$ with total measure $n$ :

$$
\mathcal{E}_{g}=\delta_{\lambda_{1}}+\cdots+\delta_{\lambda_{n}},
$$

where $\delta_{\lambda_{i}}$ is the Dirac measure in $\mathbb{C}$ with atom in $\lambda_{i}$.

Below we will pass freely between these three incarnations of the spectral measure $\mathcal{E}_{g}$ : as an element in $\mathbb{S}_{n} / \Sigma_{n}$, as an unordered tuple of $n$ complex numbers, and as a measure. 


\subsection{Continuity properties of eigenspaces}

Keeping notation as before, consider $\mathcal{E}_{g}$ as a measure and denote its support by $\left|\mathcal{E}_{g}\right|$. Given $\lambda \in\left|\mathcal{E}_{g}\right|$, ie, an eigenvalue of $g$, denote by

$$
E_{\lambda}^{g}=\operatorname{ker}(g-\lambda \mathrm{Id}) \quad \text { and } \quad \bar{E}_{\lambda}^{g}=\operatorname{ker}(g-\lambda \mathrm{Id})^{n}
$$

the $\lambda$-eigenspace and generalized $\lambda$-eigenspace of $g$. Observe that the $\mathcal{E}_{g}$-measure of the singleton $\{\lambda\}$ is given by $\mathcal{E}_{g}(\lambda)=\operatorname{dim} \bar{E}_{\lambda}^{g}$.

As $\mathbb{C}$ is algebraically closed, we have that $\mathbb{C}^{n}$ is the direct sum of the generalized eigenspaces $\bar{E}_{\lambda}^{g}$ :

$$
\mathbb{C}^{n}=\bigoplus_{\lambda \in\left|\mathcal{E}_{g}\right|} \bar{E}_{\lambda}^{g}
$$

We list a few well known linear algebraic facts:

(1) Suppose that $g, h \in \mathrm{SL}_{n} \mathbb{C}$ commute, and let $\lambda \in\left|\mathcal{E}_{g}\right|$ be an eigenvalue of $g$. Then $h E_{\lambda}^{g}=E_{\lambda}^{g}$ and $h \bar{E}_{\lambda}^{g}=\bar{E}_{\lambda}^{g}$.

(2) Suppose that $h \in \mathrm{SL}_{n} \mathbb{C}$ is such that for every $\lambda \in\left|\mathcal{E}_{g}\right|$ we have

(a) $h \bar{E}_{\lambda}^{g}=\bar{E}_{\lambda}^{g}$, and

(b) $\left.h\right|_{\bar{E}_{\lambda}^{g}} ^{g}$ is a homothety.

Then every $g^{\prime} \in \mathrm{SL}_{n} \mathbb{C}$ that commutes with $g$ also commutes with $h$, meaning that $h \in C\left(\mathcal{Z}_{\mathrm{SL}_{n} \mathbb{C}}(g)\right)$.

(3) The semisimple part $g_{s}$ of $g \in \mathrm{SL}_{n} \mathbb{C}$ is the unique linear transformation with $g_{s} v=\lambda v$ for all $v \in \bar{E}_{\lambda}^{g}$ and $\lambda \in\left|\mathcal{E}_{g}\right|$.

It is easy to find examples showing that the eigenspaces of $g$, generalized or not, do not depend continuously on $g$. The following well known lemmas show the kind of continuity we do have:

Lemma 7.3 The map $g \mapsto \mathcal{E}_{g}$ is continuous.

Proof The characteristic polynomial depends continuously on $g$; and the set of zeroes, counted with multiplicity, of a complex polynomial depends also continuously on the coefficients.

Lemma 7.4 Suppose that the sequence $\left(g_{i}\right)$ in $\mathrm{SL}_{n} \mathbb{C}$ converges to $g$. Suppose also that $\delta>0$ is such that any two distinct points in $\left|\mathcal{E}_{g}\right|$ are at distance at least $2 \delta$. Then for all $\lambda \in\left|\mathcal{E}_{g}\right|$ we have

$$
\lim _{i \rightarrow \infty} \bigoplus_{\lambda^{\prime} \in B_{\delta}(\lambda)} \bar{E}_{\lambda^{\prime}}^{g_{i}}=\bar{E}_{\lambda}^{g}
$$

Here $B_{\delta}(\lambda)$ is the ball in $\mathbb{C}$ of radius $\delta$ centered at $\lambda$. 
Sketch of proof If $\lambda_{i} \in\left|\mathcal{E}_{g_{i}}\right|$ is any sequence of eigenvalues of $g_{i}$ converging to $\lambda \in\left|\mathcal{E}_{g}\right|$, then, up to passing to a subsequence, the generalized eigenspaces $\bar{E}_{\lambda_{i}}^{g_{i}}$ converge to a subspace of $\bar{E}_{\lambda}^{g}$. It follows that

$$
\lim _{i \rightarrow \infty} \bigoplus_{\lambda^{\prime} \in B_{\delta}(\lambda)} \bar{E}_{\lambda^{\prime}}^{g_{i}} \subset \bar{E}_{\lambda}^{g}
$$

The equality follows as both spaces have the same dimension by Lemma 7.3.

\subsection{Smashing eigenvalues}

In the construction of the approximated Jordan decomposition we will need a technical lemma, proved next. Consider the following function $d: \mathbb{S}_{n} \times \mathbb{S}_{n} \rightarrow \mathbb{R}$ :

$$
d\left(\left(\begin{array}{ccc}
\lambda_{1} & & \\
& \ddots & \\
& & \lambda_{n}
\end{array}\right),\left(\begin{array}{ccc}
\lambda_{1}^{\prime} & & \\
& \ddots & \\
& & \lambda_{n}^{\prime}
\end{array}\right)\right)=\max _{i=1, \ldots, n}\left|\frac{\lambda_{i}}{\lambda_{i}^{\prime}}-1\right|
$$

The function $d(\cdot, \cdot)$ is not a distance, but we will think of it anyway as such. In this section we prove the following lemma:

Lemma 7.5 For all $\epsilon>0$ there is a $\delta \in(0, \epsilon)$ and a $\Sigma_{n}$-equivariant continuous map

$$
\Theta:\left(\mathbb{S}_{n}, \mathbb{S}_{n} \cap \mathrm{SU}_{n}\right) \rightarrow\left(\mathbb{S}_{n}, \mathbb{S}_{n} \cap \mathrm{SU}_{n}\right),
$$

$$
\Theta: \lambda=\left(\lambda_{i}\right) \mapsto \Theta(\lambda)=\left(\Theta_{i}(\lambda)\right),
$$

with $\overline{\Theta(\cdot)}=\Theta(\bar{\cdot})$ and such that for all $\lambda=\left(\lambda_{1}, \ldots, \lambda_{n}\right) \in \mathbb{S}_{n}$ and all $i, j \in\{1, \ldots, n\}$ the following holds:

(1) $d(\lambda, \Theta(\lambda)) \leq \epsilon$, and

(2) if $\left|\frac{\lambda_{i}}{\lambda_{j}}-1\right| \leq \delta$ then $\Theta_{i}(\lambda)=\Theta_{j}(\lambda)$.

Moreover, if $T_{1}, \ldots, T_{r} \subset \mathbb{S}_{n}$ are Zariski closed subgroups, then $\Theta$ can be chosen so that $\Theta\left(T_{i}\right) \subset T_{i}$ for all $i$.

Proof Let $\bar{\Sigma}_{n}$ be the finite group of transformations of $\mathbb{S}_{n}$ generated by the symmetric group $\Sigma_{n}$ and by complex conjugation $\left(\lambda_{i}\right) \mapsto\left(\bar{\lambda}_{i}\right)$. Choose a $\bar{\Sigma}_{n}$-invariant triangulation $\mathcal{T}$ of $\mathbb{S}_{n}$ that contains both $\mathbb{S}_{n} \cap \mathrm{SU}_{n}$ and the given subgroups $T_{1}, \ldots, T_{r}$ as subcomplexes, and consider $X=\mathbb{S}_{n} / \bar{\Sigma}_{n}$ with the induced structure of a simplicial 
complex. We may assume without loss of generality that for any simplex $c \in \mathcal{T}$ we have

$$
\max _{\lambda, \lambda^{\prime} \in c} d\left(\lambda, \lambda^{\prime}\right) \leq \epsilon
$$

Let now $\mathcal{U}=\left\{U_{c}\right\}_{c \in C(X)}$ be a regular covering of $X$ and choose $\delta \in\left(0, \frac{\epsilon}{2}\right)$ small enough so that

$$
\left\{x \in X \mid \min _{y \in c} d(x, y) \leq 2 \delta\right\} \subset \bigcup_{c^{\prime} \subset c} U_{c^{\prime}}
$$

for any cell $c \in C(X)$. For further use we also assume that $\delta<10^{-10}$.

Let now $\Theta^{\prime}: X \rightarrow X$ be the map provided by Lemma 5.1 and recall that $\Theta^{\prime}$ sends every closed simplex to itself. In particular, $\Theta^{\prime}$ induces uniquely a $\bar{\Sigma}_{n}$-equivariant map

$$
\Theta: \mathbb{S}_{n} \rightarrow \mathbb{S}_{n}
$$

which sends every closed simplex of $\mathcal{T}$ to itself. It follows from (7.4) that $\Theta$ satisfies (1). Notice also that as $\Theta$ maps every simplex to itself, it preserves every subcomplex in $\mathbb{S}_{n}$. It follows that $\Theta\left(\mathbb{S}_{n} \cap \mathrm{SU}_{n}\right) \subset \mathbb{S}_{n} \cap \mathrm{SU}_{n}$ and $\Theta\left(T_{i}\right) \subset T_{i}$ for all $i$.

It remains to prove that $\Phi$ satisfies (2). Given $\lambda=\left(\lambda_{1}, \ldots, \lambda_{n}\right) \in \mathbb{S}_{n}$, suppose that for some $i$ and $j$ we have

$$
\left|\frac{\lambda_{i}}{\lambda_{j}}-1\right| \leq \delta<10^{-10}
$$

and let $r \in \mathbb{C}$ be the unique complex number in the ball of radius $\frac{1}{2}$ around 1 with

$$
r^{2}=\frac{\lambda_{i}}{\lambda_{j}}
$$

We consider now the element $\lambda^{\prime}=\left(\lambda_{1}^{\prime}, \ldots, \lambda_{n}^{\prime}\right) \in \mathbb{S}_{n}$ with

$$
\begin{gathered}
\lambda_{k}^{\prime}=\lambda_{k} \quad \text { if } k \neq i, j, \\
\lambda_{i}^{\prime}=\lambda_{j}^{\prime}=r \lambda_{j},
\end{gathered}
$$

and notice that

$$
d\left(\lambda, \lambda^{\prime}\right)=\max \left\{|r-1|,\left|r^{-1}-1\right|\right\}<2 \delta .
$$

Denote by $c$ the minimal closed simplex in $\mathcal{T}$ containing $\lambda^{\prime}$ and observe that there is a cell $c^{\prime} \subset c$ with $\lambda \in U_{c^{\prime}}$ by (7.5). Since the map $\Theta$ maps $U_{c^{\prime}}$ to $c^{\prime}$, it follows that $\Theta(\lambda) \in c^{\prime} \subset c$. Observe at this point that $c$ is pointwise fixed by the stabilizer of $\lambda^{\prime}$ inside $\Sigma_{n} \subset \bar{\Sigma}_{n}$. In other words, for every $\lambda^{\prime \prime}=\left(\lambda_{1}^{\prime \prime}, \ldots, \lambda_{n}^{\prime \prime}\right) \in c$, we have 
$\lambda_{i}^{\prime \prime}=\lambda_{j}^{\prime \prime}$. This applies in particular to the point $\Theta(\lambda)$; this proves that $\Theta$ satisfies (2) and concludes the proof of Lemma 7.5.

\subsection{Proof of Proposition 7.1}

Suppose that $G_{1}, \ldots, G_{r} \subset \mathrm{SL}_{n} \mathbb{C}$ are algebraic groups, let $\mathcal{T}_{i}$ be the collection of subgroups of $\mathbb{S}_{n}$ associated by Lemma 7.2 to the subgroup $G_{i}$, and set $\mathcal{T}=\bigcup_{i} \mathcal{T}_{i}$. Suppose from now on that

$$
\Theta: \mathbb{S}_{n} \rightarrow \mathbb{S}_{n}
$$

is the map provided by Lemma 7.5 satisfying $\Theta(T) \subset T$ for all $T \in \mathcal{T}$.

Given $g \in \mathrm{SL}_{n} \mathbb{C}$ with semisimple part $g_{s}$, let $h \in \mathrm{SL}_{n} \mathbb{C}$ be such that $h g_{s} h^{-1} \in \mathbb{S}_{n}$. We define

$$
g_{s}^{\epsilon}=h^{-1}\left(\Theta\left(h g_{s} h^{-1}\right)\right) h .
$$

Notice that the equivariance of $\Theta$ under the action of the Weyl group of $\mathbb{S}^{n}$ implies that $g_{s}^{\epsilon}$ does not depend on the choice of $h$. In other words, the map

$$
\mathrm{SL}_{n} \mathbb{C} \rightarrow \mathrm{SL}_{n} \mathbb{C}, \quad g \mapsto g_{s}^{\epsilon}
$$

is well defined. Once we have defined $g_{s}^{\epsilon}$, we have no choice but to define $g_{u}^{\epsilon}$ as follows:

$$
\mathrm{SL}_{n} \mathbb{C} \rightarrow \mathrm{SL}_{n} \mathbb{C}, \quad g \mapsto g_{u}^{\epsilon}=g\left(g_{s}^{\epsilon}\right)^{-1} .
$$

Notice that by construction, $g_{s}^{\epsilon}$ and $g_{u}^{\epsilon}$ behave well with respect to conjugation, meaning that $\left(h g h^{-1}\right)_{s}^{\epsilon}=h g_{s}^{\epsilon} h^{-1}$ and $\left(h g h^{-1}\right)_{u}^{\epsilon}=h g_{u}^{\epsilon} h^{-1}$ for all $h \in \mathrm{SL}_{n} \mathbb{C}$. In other words, $g \mapsto\left(g_{s}^{\epsilon}, g_{u}^{\epsilon}\right)$ is $\mathrm{SL}_{n} \mathbb{C}$-equivariant.

Suppose now that $g, h \in \mathrm{SL}_{n} \mathbb{C}$ are such that $h g_{s} h^{-1} \in \mathbb{S}_{n}$ and notice that

$$
\bar{g}_{s}=\overline{g_{s}} \quad \text { and } \quad \bar{h} \bar{g}_{s} \bar{h}^{-1} \in \mathbb{S}_{n} .
$$

Taking into account that $\Theta$ is equivariant under complex conjugation, we have that:

$$
\begin{aligned}
\bar{g}_{s}^{\epsilon} & =\bar{h}^{-1} \Theta\left(\bar{h} \bar{g}_{s} \bar{h}^{-1}\right) \bar{h}=\bar{h}^{-1} \Theta\left(\bar{h} \bar{g}_{s} \bar{h}^{-1}\right) \bar{h} \\
& =\bar{h}^{-1} \Theta\left(\overline{h g_{s} h^{-1}}\right) \bar{h}=\bar{h}^{-1} \overline{\Theta\left(h g h^{-1}\right)} \bar{h} \\
& =\overline{h^{-1} \Theta\left(h g h^{-1}\right) h}=\overline{g_{s}^{\epsilon}} .
\end{aligned}
$$

This implies that $\bar{g}_{u}^{\epsilon}=\overline{g_{u}^{\epsilon}}$ for any $g \in \mathrm{SL}_{n} \mathbb{C}$.

Suppose now that we are given an element $g \in G_{i}$. The semisimple part $g_{s}$ also belongs to $G_{i}$. Since $g_{s}$ is diagonalizable, there is $h \in \mathrm{SL}_{n} \mathbb{C}$ with $h g_{s} h^{-1} \in \mathbb{T}$. By 
Lemma 7.2 there is $T \in \mathcal{T}_{i} \subset \mathcal{T}$ such that $h g_{s} h^{-1} \in T$. Since $\Theta$ preserves $T$ we have that $\Theta\left(h g_{s} h^{-1}\right)$ also belongs to $T$. Hence

$$
g_{s}^{\epsilon}=h^{-1} \Theta\left(h g_{s} h^{-1}\right) h \in h^{-1} T h \subset G_{i},
$$

where the last inclusion holds by Lemma 7.2. So, $g_{u}^{\epsilon}=g\left(g_{s}^{\epsilon}\right)^{-1}$ belongs to $G_{i}$ as well. A similar argument shows that $g_{s}^{\epsilon}, g_{u}^{\epsilon} \in \mathrm{SU}_{n}$ for $g \in \mathrm{SU}_{n}$.

It remains to prove that the maps $g \mapsto g_{s}^{\epsilon}$ and $g \mapsto g_{u}^{\epsilon}$ are continuous and satisfy (1), (2) and (3) in Proposition 7.1. Before doing so, we give a different description of $g_{s}^{\epsilon}$. Continuing with the same notation, identify $\mathbb{S}_{n}$ with a subset of $\mathbb{C}^{n}$ as in (7.2), let $\mathcal{E}_{g}=\left(\lambda_{1}, \ldots, \lambda_{n}\right) \in \mathbb{C}^{n}$ be the tuple associated to $h g_{s} h^{-1} \in \mathbb{S}_{n}$, and let $\left(\Theta_{1}\left(\mathcal{E}_{g}\right), \ldots, \Theta_{n}\left(\mathcal{E}_{g}\right)\right) \in \mathbb{C}^{n}$ be the tuple associated to $\Theta\left(h g_{s} h^{-1}\right)$. Then $g_{s}^{\epsilon}$ is the unique linear map with the following property

$$
g_{s}^{\epsilon}(v)=\Theta_{i}\left(\mathcal{E}_{g}\right) \cdot v \quad \text { for all } v \in \bar{E}_{\lambda_{i}}^{g} .
$$

Notice now that (7.6) implies that $g_{s}^{\epsilon}$ commutes with every element $h \in \mathrm{SL}_{n} \mathbb{C}$ that commutes with the semisimple part $g_{s}$ of $g$. In other words,

$$
g_{s}^{\epsilon} \in C\left(\mathcal{Z}_{\mathrm{SL}_{n} \mathbb{C}}\left(g_{s}\right)\right) .
$$

It follows hence from Lemma 3.8 that $g_{s}^{\epsilon}$ and its inverse are polynomials in $g_{s}$. Since $g_{s}$ is in turn a polynomial in $g$ we deduce that

$$
g_{s}^{\epsilon}, g_{u}^{\epsilon}=g\left(g_{s}^{\epsilon}\right)^{-1} \in \mathbb{C}[g] .
$$

This proves (2), and (1) follows immediately.

Starting with the proof of (3), notice that by construction, $g_{s}^{\epsilon}$ is conjugate to an element in $\mathbb{S}_{n}$ and hence is semisimple. To prove that $g_{u}^{\epsilon}$ - Id has very small spectral radius let, with the same notation as above,

$$
\mathcal{E}_{g}=\left(\lambda_{1}, \ldots, \lambda_{n}\right) \in \mathbb{C}^{n}
$$

be the tuple corresponding to $h g_{s} h^{-1} \in \mathbb{S}_{n}$. Considering $g$ in Jordan normal form we see that the eigenvalues of $g_{u}^{\epsilon}$ are given by $\lambda_{i} / \Theta_{i}\left(\mathcal{E}_{g}\right)$. It follows from Lemma 7.5 that

$$
\rho\left(g_{u}^{\epsilon}-\mathrm{Id}\right)=\max \left\{\left|\frac{\lambda_{i}}{\Theta_{i}\left(\mathcal{E}_{g}\right)}-1\right|, i=1, \ldots, n\right\}=d\left(\mathcal{E}_{g}, \Theta\left(\mathcal{E}_{g}\right)\right)<\epsilon .
$$

This proves (3).

At this point we have proven all the claims of Proposition 7.1, except for the continuity of the map $g \mapsto\left(g_{s}^{\epsilon}, g_{u}^{\epsilon}\right)=\left(g_{s}^{\epsilon}, g\left(g_{s}^{\epsilon}\right)^{-1}\right)$. It obviously suffices to prove that $g \mapsto g_{s}^{\epsilon}$ is continuous. 
Suppose that $\left(g_{i}\right)$ is a sequence in $\mathrm{SL}_{n} \mathbb{C}$ converging to some $g$. Let $\delta$ be as in Lemma 7.5, and choose $\delta_{0}<\delta$ positive, such that any two atoms of the measure $\mathcal{E}_{g}$ are at distance at least $2 \delta_{0}$. Recall that, associated to $g$ and $g_{i}$, we have direct sum decompositions of $\mathbb{C}^{n}$ into generalized eigenspaces. Given $\lambda$ in the support of $\mathcal{E}_{g}$, we define for all $i$ :

$$
V_{\lambda, i}=\bigoplus_{\substack{\lambda^{\prime} \in B_{\delta_{0}}(\lambda) \\ \mathcal{E}_{g_{i}}\left(\lambda^{\prime}\right)>0}} \bar{E}_{\lambda^{\prime}}^{g_{i}}
$$

It follows from Lemma 7.4 that

$$
\bar{E}_{\lambda}^{g}=\lim _{i \rightarrow \infty} V_{\lambda, i}
$$

for each eigenvalue $\lambda$ of $g$.

By construction the map $\left(g_{i}\right)_{S}^{\epsilon}$ is a homothety on each one of the eigenspaces of $g_{i}$. Moreover, as $\delta_{0}$ is assumed to be smaller than $\delta$, it follows from Lemma 7.5(2) that in fact the factor of the homothety of $\left(g_{i}\right)_{S}^{\epsilon}$ is equal for any two generalized eigenspaces of $g_{i}$ that are contained in the same $V_{\lambda, i}$; in other words, the restriction of $\left(g_{i}\right)_{s}^{\epsilon}$ to $V_{\lambda, i}$ is a homothety. Finally, the continuity of $\Theta$ ensures that the homothety factors of $\left(g_{i}\right)_{s}^{\epsilon}$ on $V_{\lambda, i}$ converge to the homothety factor of $g_{s}^{\epsilon}$ on $E_{\lambda}^{g}$. It follows that the maps $\left(g_{i}\right)_{s}^{\epsilon}$ converge to $g_{S}^{\epsilon}$, as claimed.

This concludes the proof of Proposition 7.1 .

Remark Notice that if $G$ is an algebraic group then we have by construction that $g_{s}^{\epsilon}=\left(g_{s}\right)_{s}^{\epsilon}$ for every $g \in G$ and every $\epsilon$ positive.

Before continuing, we wish to make a technical observation that will be important later on. Its proof, which we leave it to the reader, follows directly from the construction of the approximated Jordan decomposition:

Proposition 7.6 Suppose that $V \subset \mathbb{C}$ is a linear subspace, and suppose $g \in \mathrm{SL}_{n} \mathbb{C}$ is such that $g V=V$ and that the restriction $\left.g\right|_{V}$ of $g$ to $V$ is a homothety. If $\left(g_{j}\right)$ is a sequence of semisimple elements in $\mathrm{SL}_{n} \mathbb{C}$ converging to $g$, with

$$
g_{j} V=V
$$

for all $j$, then there is $j_{0}$ such that for all $j \geq j_{0}$ the following holds:

- $\left(g_{j}\right)_{s}^{\epsilon} V=V$, and

- the restriction $\left.\left(g_{j}\right)_{s}^{\epsilon}\right|_{V}$ of $\left(g_{j}\right)_{S}^{\epsilon}$ to $V$ is a homothety. 


\section{Retraction into $\operatorname{Hom}\left(\mathbb{Z}^{k}, K^{G}\right)$}

In this section we prove Proposition 6.1. However, before doing so we need to make a few observations on the exponential and the logarithm of a matrix.

\subsection{The exponential and the logarithm}

Recall the definition of the exponential and the logarithm as formal power series:

$$
e^{X}=\sum_{m=0}^{\infty} \frac{1}{m !} X^{m}, \quad \log (X)=\sum_{m=1}^{\infty} \frac{(-1)^{m+1}}{m}(X-\mathrm{Id})^{m} .
$$

If we substitute $X$ for an arbitrary square matrix, then the exponential series converges absolutely. The logarithmic series converges absolutely if the matrix $X-$ Id has spectral radius $\rho(X-\mathrm{Id})<1$.

Lemma 8.1 Given two commuting square matrices $g, h \in M_{n}(\mathbb{C})$ such that all the involved series converge absolutely, we have:

- $e^{\log (g)}=g$.

- $e^{g+h}=e^{g} e^{h}$.

- $\log (g h)=\log (g)+\log (h)$.

- $g$ commutes with both $e^{h}$ and $\log (h)$.

- $\overline{e^{g}}=e^{\bar{g}}$ and $\overline{\log (g)}=\log (\bar{g})$.

Aside from the simple facts listed in Lemma 8.1, we will also need the following fact, which is surely well-known to experts:

Lemma 8.2 Let $G \subset \mathrm{SL}_{n} \mathbb{C}$ be either an algebraic group or a compact group, and denote its Lie algebra by $\mathfrak{g}$. There is $\epsilon \in(0,1)$ so that $\log (g) \in \mathfrak{g}$ for all $g \in G$ with $\rho(g-$ Id $)<\epsilon$.

It is easy to construct examples showing that the constant $\epsilon$ cannot be chosen, even after fixing the ambient group $\mathrm{SL}_{n} \mathbb{C}$ and the abstract group $G$, to be independent of the embedding of $G$ in $\mathrm{SL}_{n} \mathbb{C}$.

Proof It is well-known that whenever $g \in G$ is unipotent, then $\log (g) \in \mathfrak{g}$. We consider now the semisimple case. To begin with, let $\mathcal{T}$ be the finite set of subgroups of the diagonal group $\mathbb{S}_{n} \subset \mathrm{SL}_{n} \mathbb{C}$ provided by Lemma 7.2 and for each $T_{i} \in \mathcal{T}$ let 
$\widehat{T}_{i} \subset G$ be a conjugate of $T_{i}$ and $\mathfrak{t}_{i} \subset \mathfrak{g}$ the Lie algebra of $\widehat{T}_{i}$. Since the topology of $\widehat{T}_{i}$ is the same as the induced topology, and since log is a local inverse of

$$
\mathfrak{g} \rightarrow G, \quad X \mapsto e^{X}
$$

near Id, it follows that there is small neighborhood $U \subset G$ of Id with

$$
\log \left(\widehat{T}_{i} \cap U\right) \subset \mathfrak{t}_{i}
$$

for all $i$. On the other hand, $\widehat{T}_{i}$ consists of semisimple commuting elements. Hence there is a basis $v_{1}, \ldots, v_{n}$ of $\mathbb{C}^{n}$ consisting of eigenvectors of $t \in \widehat{T}_{i}$ for all $t$. It follows that any element $t \in \widehat{T}_{i}$ with eigenvalues very close to 1 is very close to the identity. In other words, there is $\epsilon \in(0,1)$ so that for all $i$, any $t \in \widehat{T}_{i}$ with $\rho(t-\mathrm{Id})<\epsilon$ is in $U$. We have proved the claim for elements in the finite collection of subgroups $\left\{\widehat{T}_{i}\right\}$. Suppose now that $g \in G$ is an arbitrary semisimple element. By Lemma 7.2 there are $i$ and some $h \in G$ with $h g h^{-1} \in \widehat{T}_{i}$. Noticing that $\rho\left(h g h^{-1}-\mathrm{Id}\right)<\epsilon$ whenever $\rho(g-\mathrm{Id})<\epsilon$, we deduce that

$$
\log (g)=h^{-1} \log \left(h g h^{-1}\right) h=\operatorname{Ad}_{h^{-1}}\left(\log \left(h g h^{-1}\right)\right) \in \operatorname{Ad}_{h^{-1}} \mathfrak{t}_{i} \subset \mathfrak{g}
$$

for any $g \in G$ semisimple with $\rho(g-$ Id $)<\epsilon$.

To conclude the proof we consider now the case of a general element $g \in G$ with $\rho(g-\mathrm{Id})<\epsilon$. Consider the Jordan decomposition $g=g_{s} g_{u}$ of $g$, and notice that $\rho\left(g_{s}-\mathrm{Id}\right)=\rho(g-\mathrm{Id})<\epsilon<1$, and that $\rho\left(g_{u}-\mathrm{Id}\right)=0$. Hence, $\log \left(g_{s}\right)$ and $\log \left(g_{u}\right)$ are also absolutely converging power series. Since $g_{s}$ and $g_{u}$ commute, and since $\log \left(g_{u}\right), \log \left(g_{s}\right) \in \mathfrak{g}$, we deduce from Lemma 8.1 that

$$
\log (g)=\log \left(g_{s}\right)+\log \left(g_{u}\right) \in \mathfrak{g} .
$$

This concludes the proof of Lemma 8.2.

From Lemma 8.2 we obtain immediately the following corollary:

Corollary 8.3 Let $G \subset \mathrm{SL}_{n} \mathbb{C}$ be either an algebraic group or a compact group. There is $\epsilon \in(0,1)$ with $e^{t \log (g)} \in G$ for all $g \in G$ with $\rho(g-\mathrm{Id})<\epsilon$ and all $t$.

\subsection{Retraction on the level of $G$}

Recall that for $n$ sufficiently large, there is no retraction of $\mathrm{SL}_{n} \mathbb{C}$ to $\mathrm{SU}_{n}$ preserving commutativity [34]. What we prove now is that if $G \subset \mathrm{SL}_{n} \mathbb{C}$ is an algebraic group, then there is a weak retraction, preserving commutativity, of $G$ into the set $G^{s}$ of semisimple elements in $G$. 
Proposition 8.4 Suppose that $G \subset \mathrm{SL}_{n} \mathbb{C}$ is an algebraic group, and let $K \subset G$ be a maximal compact subgroup. For all $\epsilon>0$ small enough, there is a $G$-equivariant continuous map

$$
F:[0,1] \times(G, K) \rightarrow(G, K), \quad(t, g) \mapsto F_{t}(g)
$$

with

$$
F_{0}(g)=g \quad \text { and } \quad F_{1}(g)=g_{s}^{\epsilon}
$$

for all $g \in G$, where $g_{s}^{\epsilon}$ is as provided by Theorem 1.7. Moreover, if $G$ is defined over $\mathbb{R}$, then $F$ is equivariant under complex conjugation.

In particular,

- $F_{1}(G) \subset G^{s}$, and

- if $g, h \in G$ commute then so do $F_{t}(g)$ and $F_{t}(h)$ for all $t$.

Here, as always, $G$ acts on itself by conjugation.

Proof Suppose that $\epsilon$ is positive and smaller than the constant provided by Corollary 8.3 for the groups $G$ and $K$. For $g \in G$ consider the approximated Jordan decomposition $g=g_{s}^{\epsilon} g_{u}^{\epsilon}$ provided by Theorem 1.7 and recall that $\rho\left(g_{u}^{\epsilon}-\mathrm{Id}\right)<\epsilon$. We define the map $F$ as follows:

$$
F:[0,1] \times G \rightarrow \mathrm{SL}_{n} \mathbb{C}, \quad F_{t}(g)=g_{s}^{\epsilon} e^{(1-t) \cdot \log g_{u}^{\epsilon}} .
$$

The $G$-equivariance and continuity of $F$ follow directly from Theorem 1.7 and the absolute convergence of the involved series. As $g_{s}^{\epsilon}, g_{u}^{\epsilon} \in G$, we deduce from the choice of $\epsilon$ and Corollary 8.3 that $F_{t}(g)$ belongs to $G$ for all $t$. The same argument implies that whenever $g \in K$ we have $F_{t}(g) \in K$ for all $t$. Also, if $G$ is defined over $\mathbb{R}$, then equivariance of the approximated Jordan decomposition under complex conjugation implies that $F$ is equivariant as well.

Notice that the $G$-equivariance of $F$ implies that if $g, h \in G$ commute then so do $F_{t}(g)$ and $h$ for all $t$. This implies that $F_{t}(g)$ and $F_{t}(h)$ also commute for all $t$.

\subsection{Scaling}

As above, let $\mathbb{S}_{n}$ be the diagonal group of $\mathrm{SL}_{n} \mathbb{C}$, identified with a subset of $\mathbb{C}^{n}$ as in (7.2). Consider the map

$$
\bar{\sigma}:[0,1] \times \mathbb{S}_{n} \rightarrow \mathbb{S}_{n}, \quad\left(t,\left(\lambda_{i}\right)\right) \mapsto \bar{\sigma}_{t}\left(\left(\lambda_{i}\right)\right)=\left(e^{-t \log \left|\lambda_{i}\right|} \lambda_{i}\right),
$$


where $\log (\cdot)$ is the standard real $\operatorname{logarithm}$. Notice that $\bar{\sigma}$ is continuous and equivariant under the action of the Weyl group $\Sigma_{n}$, and that we have

$$
\bar{\sigma}_{0}(g)=g \quad \text { and } \quad \bar{\sigma}_{1}(g) \in \mathbb{S}_{n} \cap \mathrm{SU}_{n}
$$

for all $g \in \mathbb{S}_{n}$.

Given now $g \in \mathrm{SL}_{n} \mathbb{C}$ semisimple, there is $h$ with $h g h^{-1} \in \mathbb{S}_{n}$. Invariance of $\bar{\sigma}$ under the Weyl group implies that for all $t$ the element

$$
\sigma_{t}(g)=h^{-1} \bar{\sigma}_{t}\left(h g h^{-1}\right) h
$$

is independent of the choice of $h$. In particular, we obtain a well defined map

$$
\sigma:[0,1] \times \mathrm{SL}_{n}^{s} \mathbb{C} \rightarrow \mathrm{SL}_{n}^{s} \mathbb{C}, \quad(t, g) \mapsto \sigma_{t}(g),
$$

where $\mathrm{SL}_{n}^{s} \mathbb{C}$ is the set of semisimple elements in $\mathrm{SL}_{n} \mathbb{C}$. It is easy to see that $\sigma$ is continuous and that it satisfies the following properties:

(1) $\sigma_{0}(g)=g$ and $\sigma_{1}(g) \in \bigcup_{h \in \mathrm{SL}_{n} \mathbb{C}} h \mathrm{SU}_{n} h^{-1}$ for all $g \in \mathrm{SL}_{n}^{s} \mathbb{C}$.

(2) $\sigma_{t}(g)=g$ for all $g \in \mathrm{SU}_{n}$.

(3) $\sigma$ is $\mathrm{SL}_{n} \mathbb{C}$-equivariant; in particular, if $g$ and $h$ commute, then so do $\sigma_{t}(g)$ and $\sigma_{t}(h)$ for all $t$.

(4) If $g \in \mathrm{SL}_{n} \mathbb{R}$, then $\sigma_{t}(g) \in \mathrm{SL}_{n} \mathbb{R}$ for all $t$.

Suppose now that $G \subset \mathrm{SL}_{n} \mathbb{C}$ is an algebraic group, let $\mathcal{T}$ be finite collection of subgroups of the diagonal groups $\mathbb{S}_{n} \subset \mathrm{SL}_{n} \mathbb{C}$ provided by Lemma 7.2, and let $K$ be a maximal compact subgroup in $G$. Up to conjugating in $\mathrm{SL}_{n} \mathbb{C}$, we can assume that $K \subset \mathrm{SU}_{n}$. By Lemma 7.2, every semisimple element $g \in G$ there is $h \in \mathrm{SL}_{n} \mathbb{C}$ with $h g h^{-1} \in T_{i}$ for some $i$. Since the map $\bar{\sigma}_{t}(\cdot)$ preserves $T_{i}$ for all $t$, and since $h^{-1} T_{i} h \subset G$, we deduce from that $\sigma_{t}(g) \in G$ for all $t$ and all $g \in G$ semisimple. With notation as in (2.1), we have hence proved:

Lemma 8.5 Suppose that $G$ is a linear algebraic group, and let $K \subset G$ be a maximal compact subgroup. There is a $G$-equivariant continuous map

$$
\sigma:[0,1] \times G^{s} \rightarrow G^{s}, \quad(t, g) \mapsto \sigma_{t}(g)
$$

with:

(1) $\sigma_{0}(g)=g$ and $\sigma_{1}(g) \in K^{G}$ for all $g \in G^{s}$.

(2) $\sigma_{t}(g)=g$ for all $t$ and all $g \in K^{G}$.

In particular, if $g, h \in G$ commute, then so do $\sigma_{t}(g)$ and $\sigma_{t}(h)$ for all $t$.

Moreover, if $G$ is defined over $\mathbb{R}$, then $\sigma$ is equivariant under complex conjugation. 


\subsection{Proof of Proposition 6.1}

At this point we are finally ready to prove:

Proposition 6.1 Let $G$ be the group of complex points of a reductive algebraic group, $K$ a maximal compact subgroup of $G$, and recall that $G$ acts on $\operatorname{Hom}\left(\mathbb{Z}^{k}, G\right)$ by conjugation. There is a continuous $G$-equivariant map

$\Phi:[0,1] \times\left(\operatorname{Hom}\left(\mathbb{Z}^{k}, G\right), \operatorname{Hom}\left(\mathbb{Z}^{k}, K\right)\right) \rightarrow\left(\operatorname{Hom}\left(\mathbb{Z}^{k}, G\right), \operatorname{Hom}\left(\mathbb{Z}^{k}, K\right)\right)$

with the following properties:

- $\Phi(0, \rho)=\rho$ and $\Phi(1, \rho) \in \operatorname{Hom}\left(\mathbb{Z}^{k}, K^{G}\right)$ for all $\rho \in \operatorname{Hom}\left(\mathbb{Z}^{k}, G\right)$.

- There is an open covering $\mathcal{W}$ of $\operatorname{Hom}\left(\mathbb{Z}^{k}, K^{G}\right)$ such that for every $W \in \mathcal{W}$ one of the following non-exclusive alternatives holds:

(1) All the elements in $\Phi(\{1\} \times W)$ have the same orbit type.

(2) All the elements in $W$ have the same orbit type.

If moreover $G$ is defined over $\mathbb{R}$, then $\Phi$ is equivariant under complex conjugation.

Recall that two elements $\rho, \rho^{\prime}$ in $\operatorname{Hom}\left(\mathbb{Z}^{k}, G\right)$ have the same orbit type if their stabilizers $\operatorname{Stab}_{G}(\rho)$ and $\operatorname{Stab}_{G}\left(\rho^{\prime}\right)$ are conjugate in $G$.

Proof Choose $\epsilon>0$ small enough for Proposition 8.4 to apply, and let

$$
F:[0,1] \times G \rightarrow G, \quad(t, g) \mapsto F_{t}(g)
$$

be the map provided by that proposition. Recall that $F_{1}(g)=g_{s}^{\epsilon}$, where $g_{s}^{\epsilon} \in G^{s}$ is the approximated semisimple part of $g$ provided by Theorem 1.7. Let also

$$
\sigma:[0,1] \times G^{s} \rightarrow G^{s}, \quad(t, g) \mapsto \sigma_{t}(g)
$$

be the map provided by Lemma 8.5 .

Considering elements in $\operatorname{Hom}\left(\mathbb{Z}^{k}, G\right)$ as commuting $k$-tuples in $G$, we define $\Phi$ as follows:

$$
\begin{array}{ll}
\Phi\left(t,\left(a_{1}, \ldots, a_{k}\right)\right)=\left(F_{2 t}\left(a_{1}\right), \ldots, F_{2 t}\left(a_{k}\right)\right) & \text { for } t \leq \frac{1}{2} \\
\Phi\left(t,\left(a_{1}, \ldots, a_{k}\right)\right)=\left(\sigma_{2 t-1}\left(\left(a_{1}\right)_{s}^{\epsilon}\right), \ldots, \sigma_{2 t-1}\left(\left(a_{k}\right)_{s}^{\epsilon}\right)\right) & \text { for } t \geq \frac{1}{2} .
\end{array}
$$

By construction, the homotopy $\Phi$ is continuous, preserves $\operatorname{Hom}\left(\mathbb{Z}^{k}, K\right)$, and we have $\Phi(0, \rho)=\rho$ and $\Phi(1, \rho) \in \operatorname{Hom}\left(\mathbb{Z}^{k}, K^{G}\right)$ for all $\rho \in \operatorname{Hom}\left(\mathbb{Z}^{k}, G\right)$. Moreover, it follows from the $G$-equivariance of $F$ and $\sigma$ that $\Phi$ is $G$-equivariant as well. Also, 
if $G$ is defined over $\mathbb{R}$, then $\Phi$ is equivariant under complex conjugation because $F$ and $\sigma$ are.

We have proved all claims of Proposition 6.1 but the existence of the covering $\mathcal{W}$. Suppose now that $H \subset G$ is a subgroup, and consider the set

$$
\mathcal{X}_{H}=\left\{\rho \in \operatorname{Hom}\left(\mathbb{Z}^{k}, K^{G}\right) \mid \exists g \in G \text { with } g H g^{-1} \subset \operatorname{Stab}_{G}(\rho)\right\} .
$$

It follows from Proposition 2.8 that there is a triangulation of $\operatorname{Hom}\left(\mathbb{Z}^{k}, K^{G}\right)$ such that $\mathcal{X}_{H}$ is a (closed) subcomplex for all $H \subset G$.

Noting that $G$-equivariance implies $\Phi\left(1, \mathcal{X}_{H}\right) \subset \mathcal{X}_{H}$, observe that the existence of the open cover $\mathcal{W}$ follows once we have established the following claim:

Claim For every $H \subset G$ there is an open neighborhood $U$ of $\mathcal{X}_{H}$ with $\Phi(1, U) \subset \mathcal{X}_{H}$.

Proof of the claim Given a sequence $\left(a_{1}^{j}, \ldots, a_{k}^{j}\right)$ in $\operatorname{Hom}\left(\mathbb{Z}^{k}, K^{G}\right)$ converging to $\left(a_{1}, \ldots, a_{k}\right) \in \mathcal{X}_{H}$, notice that it follows from Theorem 2.7, Theorem 2.4 and the remark after the latter theorem, that there is a sequence $\left(g_{j}\right)$ in $G$ converging to $\operatorname{Id}_{G}$ and with

$$
\begin{aligned}
\operatorname{Stab}_{G}\left(g_{j} a_{1}^{j} g_{j}^{-1}, \ldots, g_{j} a_{k}^{j} g_{j}^{-1}\right) & =g_{j} \operatorname{Stab}_{G}\left(a_{1}^{j}, \ldots, a_{k}^{j}\right) g_{j}^{-1} \\
& \subset \operatorname{Stab}_{G}\left(a_{1}, \ldots, a_{k}\right)
\end{aligned}
$$

for all sufficiently large $j$. Observe also that by the $G$-equivariance of $\Phi$ and the $G$-invariance of $\mathcal{X}_{H}$, it suffices to prove that

$$
\Phi\left(1, g_{j} a_{1}^{j} g_{j}^{-1}, \ldots, g_{j} a_{k}^{j} g_{j}^{-1}\right) \in \mathcal{X}_{H}
$$

for all $j$ large enough. We may hence assume, without loss of generality, that

$$
\left(a_{1}^{j}, \ldots, a_{k}^{j}\right) \rightarrow\left(a_{1}, \ldots, a_{k}\right), \quad \operatorname{Stab}_{G}\left(a_{1}^{j}, \ldots, a_{k}^{j}\right) \subset \operatorname{Stab}_{G}\left(a_{1}, \ldots, a_{k}\right) \supset H
$$

for all $j$.

By assumption, the elements $a_{1}, \ldots, a_{k} \in G \subset \mathrm{SL}_{n} \mathbb{C}$ commute and are diagonalizable. In particular, they are simultaneously diagonalizable. Let

$$
\mathbb{C}^{n}=V_{1} \oplus \cdots \oplus V_{r}
$$

be the coarsest direct sum decomposition of $\mathbb{C}^{n}$ such that for all $i=1, \ldots, k$ and all $s=1, \ldots, r$ we have:

- $a_{i}\left(V_{s}\right)=V_{s}$, and

- the restriction $\left.a_{i}\right|_{V_{s}}$ of $a_{i}$ to $V_{s}$ is a homothety. 
Notice that the assumption that $\mathbb{C}^{n}=V_{1} \oplus \cdots \oplus V_{r}$ is the coarsest direct sum decomposition with these properties implies that

$$
\operatorname{Stab}_{G}\left(a_{1}, \ldots, a_{k}\right)=G \cap\left(\mathrm{GL}\left(V_{1}\right) \times \cdots \times \mathrm{GL}\left(V_{r}\right)\right) .
$$

For sufficiently large $j$ we have hence that the elements

$$
a_{1}^{j}, \ldots, a_{k}^{j} \in \operatorname{Stab}_{G}\left(a_{1}^{j}, \ldots, a_{k}^{j}\right) \subset \operatorname{Stab}_{G}\left(a_{1}, \ldots, a_{k}\right)
$$

preserve $V_{1}, \ldots, V_{r}$. Proposition 7.6 implies that for all $i$ and $s$, and for all sufficiently large $j$, we have

- $\left(a_{i}^{j}\right)_{s}^{\epsilon} V_{s}=V_{s}$, and

- the restriction $\left.\left(a_{i}^{j}\right)_{s}^{\epsilon}\right|_{V_{s}}$ of $\left(a_{i}^{j}\right)_{s}^{\epsilon}$ to $V_{s}$ is a homothety.

This implies that $\mathcal{Z}_{G}\left(\left(a_{i}^{j}\right)_{s}^{\epsilon}\right) \supset G \cap\left(\mathrm{GL}\left(V_{1}\right) \times \cdots \times \mathrm{GL}\left(V_{r}\right)\right)$ for all $j$. At this point notice that the $G$-equivariance of the map $\sigma$ provided by Lemma 8.5 implies that we also have

$$
\mathcal{Z}_{G}\left(\sigma_{1}\left(\left(a_{i}^{j}\right)_{s}^{\epsilon}\right)\right) \supset G \cap\left(\operatorname{GL}\left(V_{1}\right) \times \cdots \times \operatorname{GL}\left(V_{r}\right)\right)
$$

for all $j$. This shows that

$$
\begin{aligned}
\operatorname{Stab}_{G}\left(\Phi\left(1,\left(a_{1}^{j}, \ldots, a_{k}^{j}\right)\right)\right) & =\operatorname{Stab}_{G}\left(\sigma_{1}\left(\left(a_{1}^{j}\right)_{s}^{\epsilon}\right), \ldots, \sigma_{1}\left(\left(a_{k}^{j}\right)_{s}^{\epsilon}\right)\right) \\
& =\bigcap_{i=1}^{j} \mathcal{Z}_{G}\left(\left(a_{i}^{j}\right)_{s}^{\epsilon}\right) \\
& \supset G \cap\left(\operatorname{GL}\left(V_{1}\right) \times \cdots \times \operatorname{GL}\left(V_{r}\right)\right) \\
& =\operatorname{Stab}_{G}\left(a_{1}, \ldots, a_{k}\right) \supset H
\end{aligned}
$$

implying that $\Phi\left(1,\left(a_{1}^{j}, \ldots, a_{k}^{j}\right)\right) \in \mathcal{X}_{H}$, as we needed to prove.

Having established the claim, we have proved Proposition 6.1.

\section{Appendix}

While reading this appendix the reader should always keep in mind the terminology and notation introduced in Section 5. More concretely, notation will always be as in $(*)$ in Section 5 .

We will identify four properties of the set $(\mathcal{R}, \mathcal{O})$ of fibered relation pairs that ensure that if $(X, \delta)$ is a $\mathcal{P}$-labeled complex and $\mathcal{U}$ is a regular cover of $X$, then every section of $\mathcal{S} \times{ }_{\delta} \mathcal{U}$ is homotopic to a section of $\mathcal{K} \times{ }_{\delta} \mathcal{U} \subset \mathcal{S} \times{ }_{\delta} \mathcal{U}$. More precisely, we will 
define transitivity, reflexivity, and the simultaneous lifting and the relative retraction properties so that the following holds:

Proposition A.1 With notation as in (*), suppose that $\mathcal{R}$ is transitive and reflexive, that it satisfies the simultaneous lifting property, and that $(\mathcal{R}, \mathcal{O})$ satisfies the relative retraction property. Let also $(X, \delta)$ be a $\mathcal{P}$-labeled locally finite and finite-dimensional simplicial complex, and $\mathcal{U}=\left\{U_{c}\right\}_{c \in C(X)}$ and $\mathcal{V}=\left\{V_{c}\right\}_{c \in C(X)}$ regular covers of $X$ with $V_{c}$ relatively compact in $U_{c}$ for all $c \in C(X)$. Finally, let $\left\{\sigma_{c}\right\}_{c \in C(X)}$ be a section of $\mathcal{S} \times{ }_{\delta} \mathcal{U}$, and $W \subset X$ with $\sigma_{c}(w) \in K_{\delta(c)}$ for all $c \in C(X)$ and all $x \in U_{c} \cap W$.

The restriction of $\left\{\sigma_{c}\right\}_{c \in C(X)}$ to $\mathcal{S} \times_{\delta} \mathcal{V}$ is homotopic to a section $\left\{\sigma_{c}^{\prime}\right\}_{c \in C(X)}$ with $\sigma_{c}^{\prime}\left(V_{c}\right) \subset K_{\delta(c)}$ for all $c \in C(X)$; moreover the homotopy is constant on $W$.

Once Proposition A.1 is proved, we will show that the sets of fibered relation pairs $\left(\mathcal{R}_{G}, \mathcal{O}_{G}\right)$ associated in (5.1) and (5.2) to a group $G$ satisfies these four properties. Proposition 5.3 follows immediately.

\section{A.1 Transitivity and reflexivity}

The set $\mathcal{R}$ is transitive if

$$
\mathcal{R}_{\Delta, \Delta^{\prime \prime}} \supset\left\{(x, z) \in S_{\Delta} \times S_{\Delta^{\prime \prime}} \mid \exists y \in S_{\Delta^{\prime}} \text { with }(x, y) \in \mathcal{R}_{\Delta, \Delta^{\prime}},(y, z) \in \mathcal{R}_{\Delta^{\prime}, \Delta^{\prime \prime}}\right\}
$$

for all $\Delta, \Delta^{\prime}, \Delta^{\prime \prime} \in \mathcal{P}$ with $\Delta \geq \Delta^{\prime} \geq \Delta^{\prime \prime} ; \mathcal{R}$ is reflexive if

$$
\mathcal{R}_{\Delta, \Delta}=\left\{(x, x) \in S_{\Delta} \times S_{\Delta} \mid x \in S_{\Delta}\right\}
$$

for all $\Delta \in \mathcal{P}$.

Notice that transitivity and reflexivity of $\mathcal{R}$ imply that $\mathcal{O}$ is also transitive and reflexive.

\section{A.2 Simultaneous lifting}

Suppose now that $(X, \delta)$ is a $\mathcal{P}$-labeled simplicial complex, and let $\mathcal{U}=\left(U_{c}\right)_{c \in C(X)}$ be a regular cover. Consider the bundle

$$
\mathcal{S} \times{ }_{\delta} \mathcal{U}:=\bigsqcup_{c \in C(X)}\left(S_{\delta(c)} \times U_{c}\right) \rightarrow \bigsqcup_{c \in C(X)} U_{c}
$$

Our goal is to prove that, under certain conditions, one can homotope sections $\left\{\sigma_{c}\right\}_{c \in C(X)}$ of $\mathcal{S} \times_{\delta} \mathcal{U}$ to sections $\left\{\sigma_{c}^{\prime}\right\}_{c \in C(X)}$ such that $\sigma_{c}^{\prime}$ takes values in the subset $K_{\delta(c)} \subset S_{\delta(c)}$ for all $c \in C(X)$. In order to do so we want to be able to construct homotopies of sections skeleton by skeleton, ie, starting with the maps $\sigma_{c}: U_{c} \rightarrow \mathcal{S}_{\delta(c)}$ 
corresponding to the vertices of $X$, continuing with those corresponding to the edges, etc... We will see shortly that the following is the property allowing us to do so.

The set $\mathcal{R}$ has the simultaneous lifting property if:

(SL) Suppose that $\Delta_{0}, \Delta_{1}, \ldots, \Delta_{r} \in \mathcal{P}$ are such that $\Delta_{i} \geq \Delta_{0}$ for all $i$ and that $W_{0}, \ldots, W_{r}$ are contractible open sets in a CW-complex with $W_{i} \subset W_{0}$ for all $i$. Finally suppose that we have for each $i=0, \ldots, r$ a continuous map $\sigma_{i}: W_{i} \rightarrow S_{\Delta_{i}}$ with $\left(\sigma_{i}(x), \sigma_{j}(x)\right) \in \mathcal{R}_{\Delta_{i}, \Delta_{j}}$ for all $i, j \in\{0, \ldots, r\}$ with $\Delta_{i} \geq \Delta_{j}$ and all $x \in W_{i} \cap W_{j}$.

For any continuous map $\phi_{0}:[0,1] \times W_{0} \rightarrow S_{\Delta_{0}}$ with $\phi_{0}(0, x)=\sigma_{0}(x)$ there are continuous maps $\phi_{i}:[0,1] \times W_{i} \rightarrow \mathcal{S}_{\Delta_{i}}$ for $i=1, \ldots, r$ with $\phi_{i}(0, x)=\sigma_{i}(x)$ and with

$$
\left(\phi_{i}(t, x), \phi_{j}(t, x)\right) \in \mathcal{R}_{\Delta_{i}, \Delta_{j}}
$$

for all $t$, all $i, j \in\{0, \ldots, r\}$ with $\Delta_{i} \geq \Delta_{j}$ and all $x \in W_{i} \cap W_{j}$.

Moreover, if the homotopy $\phi_{0}$ is constant outside of some compact set $C \subset W_{0}$, then $\phi_{i}$ is also constant outside of $C \cap W_{i}$.

Lemma A.2 (Lifting Lemma) Suppose that $\mathcal{R}$ is transitive and reflexive, and satisfies the simultaneous lifting property and let $\mathcal{U}=\left\{U_{c}\right\}_{c \in C(X)}$ be a regular cover of a $\mathcal{P}$ labeled complex $(X, \delta)$.

Suppose moreover that $\left\{\sigma_{c}\right\}_{c \in C(X)}$ is a section of $\mathcal{S} \times{ }_{\delta} \mathcal{U}$ and that for some $c_{0} \in C(X)$ we have a continuous map

$$
[0,1] \times U_{c_{0}} \rightarrow \mathcal{S}_{\delta\left(c_{0}\right)}, \quad(t, x) \rightarrow \bar{\sigma}_{c_{0}}^{t}(x)
$$

with $\bar{\sigma}_{c_{0}}^{0}=\sigma_{c_{0}}$, such that:

(1) $\left(\bar{\sigma}_{c_{0}}^{t}(x), \sigma_{c}(x)\right) \in \mathcal{R}_{\delta\left(c_{0}\right), \delta(c)}$ for all $c_{0} \supsetneq c$ and all $x \in U_{c_{0}} \cap U_{c}$.

(2) $\bar{\sigma}_{c_{0}}^{t}(x)=\sigma_{c_{0}}(x)$ for all $x \notin C$, where $C \subset U_{c_{0}}$ is compact.

Then there is a homotopy of sections $\left\{\sigma_{c}^{t}\right\}_{c \in C(X)}$ of $\mathcal{S} \times_{\delta} \mathcal{U}$ satisfying

- $\sigma_{c}^{0}=\sigma_{c}$ for all $c \in C(X)$,

- $\sigma_{c_{0}}^{t}=\bar{\sigma}_{c_{0}}^{t}$ for all $t$,

- $\sigma_{c}^{t}=\sigma_{c}$ for all $c \in C(X)$ with $c_{0} \subsetneq c$ and all $t$, and

- the homotopy $\left\{\sigma_{c}^{t}\right\}_{c \in C(X)}$ is constant on $X \backslash C$. 
Proof Let $c_{1}, \ldots, c_{s} \in C(X) \backslash\left\{c_{0}\right\}$ be cells in $X$ with $U_{c_{i}} \cap U_{c_{0}} \neq \varnothing$ and observe that these are exactly the cells $c_{i}$ in $C(X)$ with either $c_{0} \subsetneq c_{i}$ or with $c_{i} \subsetneq c_{0}$. We may assume that $c_{1}, \ldots, c_{r}$ are those containing $c_{0}$ and $c_{r+1}, \ldots, c_{s}$ those contained in $c_{0}$. In particular $\delta\left(c_{i}\right) \geq \delta\left(c_{0}\right)$ for all $i=1, \ldots, r$. Applying the simultaneous lifting property to

- $\delta\left(c_{0}\right), \ldots, \delta\left(c_{r}\right)$,

- the contractible open sets $U_{c_{i}} \cap U_{c_{0}}$ for $i=0, \ldots, r$,

- the restrictions of $\sigma_{c_{i}}$ to $U_{c_{i}} \cap U_{c_{0}}$ again for $i=0, \ldots, r$, and

- the map $[0,1] \times U_{c_{0}} \rightarrow S_{\delta\left(c_{0}\right)},(t, x) \mapsto \bar{\sigma}_{c_{0}}^{t}(x)$,

we obtain for $i=1, \ldots, r$ a map

$$
[0,1] \times\left(U_{c_{0}} \cap U_{c_{i}}\right) \rightarrow S_{\delta\left(c_{i}\right)}, \quad(t, x) \mapsto \bar{\sigma}_{c_{i}}^{t}(x)
$$

satisfying $\left(\bar{\sigma}_{c_{i}}^{t}(x), \bar{\sigma}_{c_{j}}^{t}(x)\right) \in \mathcal{R}_{\delta(i), \delta(j)}$ for all $i, j \in\{0, \ldots, r\}$ with $c_{i} \supset c_{j}$ and all $x \in U_{c_{i}} \cap U_{c_{j}}$. A priori, the maps $\bar{\sigma}_{c_{i}}^{t}$ are only defined on $U_{c_{i}} \cap U_{c_{0}}$. However, since $\bar{\sigma}_{c_{0}}^{t}(x)=\sigma_{c_{0}}(x)$ for all $x \in U_{c_{0}} \backslash C$, we can extend $\bar{\sigma}_{c_{i}}^{t}$ continuously to $U_{c_{i}}$ by $\bar{\sigma}_{c_{i}}^{t}(x)=\sigma_{c_{i}}(x)$ for all $x \in U_{c_{i}} \backslash C$.

Suppose now that $c_{i} \subsetneq c_{0}$, ie, $i \in\{s+1, \ldots, r\}$. By the assumptions in the lemma we have that $\left(\bar{\sigma}_{c_{0}}^{t}(x), \sigma_{c_{i}}(x)\right) \in \mathcal{R}_{\delta\left(c_{0}\right), \delta\left(c_{i}\right)}$ for all $x \in U_{c_{0}} \cap U_{c_{i}}$. The transitivity of $\mathcal{R}$ implies thus that for all $j \in\{1, \ldots, r\}$ we have also $\left(\bar{\sigma}_{c_{j}}^{t}(x), \sigma_{c_{i}}(x)\right) \in \mathcal{R}_{\delta\left(c_{j}\right), \delta\left(c_{i}\right)}$ for all $x \in U_{c_{j}} \cap U_{c_{j}}$. It follows that $\left\{\sigma_{c}^{t}\right\}_{c \in C(X)}$ with

$$
\sigma_{c}^{t}=\bar{\sigma}_{c}^{t} \quad \text { for } c \in\left\{c_{0}, \ldots, c_{r}\right\}, \quad \sigma_{c}^{t}=\sigma_{c} \quad \text { for } c \notin\left\{c_{0}, \ldots, c_{r}\right\}
$$

is a section of $\mathcal{S} \times{ }_{\delta} \mathcal{U}$ for all $t$. It has the desired properties by construction.

\section{A.3 Relative retractions}

We will be interested later in pairs $\left(S_{\Delta}, K_{\Delta}\right)$ with the property that the inclusion $K_{\Delta} \rightarrow S_{\Delta}$ is a homotopy equivalence. However, since we are not working with single spaces but with sets of spaces, we need that the homotopy equivalence between $K_{\Delta}$ and $S_{\Delta}$ behaves well with respect to other pairs $\left(S_{\Delta^{\prime}}, K_{\Delta^{\prime}}\right)$.

The set $(\mathcal{R}, \mathcal{O})$ satisfies the relative retraction property if:

(RR1) For all $\Delta \in \mathcal{P}$ the inclusion $K_{\Delta} \rightarrow S_{\Delta}$ is a homotopy equivalence.

(RR2) For all $\Delta, \Delta^{\prime} \in \mathcal{P}$ with $\Delta \geq \Delta^{\prime}$ there is a retraction of $\left.\mathcal{R}_{\Delta, \Delta^{\prime}} \cap\left(S_{\Delta} \times K_{\Delta^{\prime}}\right)\right)$ to $\mathcal{O}_{\Delta, \Delta^{\prime}}=\mathcal{R}_{\Delta, \Delta^{\prime}} \cap\left(K_{\Delta} \times K_{\Delta^{\prime}}\right)$ that preserves the fibers of the projection $\left.\mathcal{R}_{\Delta, \Delta^{\prime}} \cap\left(S_{\Delta} \times K_{\Delta^{\prime}}\right)\right) \rightarrow K_{\Delta^{\prime}}$. 
We formulate the second condition in more concrete, but also more obscure terms:

Lemma A.3 (Reformulation of (RR2)) Suppose that $(\mathcal{R}, \mathcal{O})$ satisfies the relative retraction property. Given $\Delta_{0}, \Delta_{1} \in \mathcal{P}$ with $\Delta_{0} \geq \Delta_{1}$, an open set $W$ in a $C W$-complex, continuous maps $\sigma_{i}: W \rightarrow S_{\Delta_{i}}$ with $\left(\sigma_{0}(x), \sigma_{1}(x)\right) \in \mathcal{R}_{\Delta_{0}, \Delta_{1}}$ for all $x \in W$ and such that $\sigma_{1}(W) \subset K_{\Delta_{1}}$, and finally $U \subset W$ open and relatively compact, then the following holds:

There is a continuous map $\phi_{0}:[0,1] \times W \rightarrow \mathcal{S}_{\Delta_{0}}$ satisfying:

(1) $\phi_{0}(0, x)=\sigma_{0}(x)$ for all $x \in W$,

(2) $\left(\phi_{0}(t, x), \sigma_{1}(x)\right) \in \mathcal{R}_{\Delta_{0}, \Delta_{1}}$ for all $x \in W$ and all $t$,

(3) $\phi_{0}(t, x)=\sigma_{0}(x)$ for all $x$ outside some compact set $C \subset W$ and all $x \in W$ with $\sigma_{0}(x) \in K_{\Delta_{0}}$, and

(4) $\phi_{0}(1, x) \in K_{\Delta_{0}}$ for all $x \in U$.

Before the relative retraction property can be of any use to us, we need to extract from it something that takes into account the interplay between multiple members of the set $(\mathcal{R}, \mathcal{O})$ of fibered relations, and not only two of them. This is done in the following lemma:

Lemma A.4 (Retraction Lemma) Suppose that $\mathcal{R}$ is transitive and reflexive, and that $(\mathcal{R}, \mathcal{O})$ satisfies the relative retraction property. Let $\mathcal{U}=\left\{U_{c}\right\}_{c \in C(X)}$ and $\mathcal{V}=$ $\left\{V_{c}\right\}_{c \in C(X)}$ be regular covers of a $\mathcal{P}$-labeled complex $(X, \delta)$ with $V_{c}$ relatively compact in $U_{c}$ for all $c \in C(X)$.

Suppose moreover that $\left\{\sigma_{c}\right\}_{c \in C(X)}$ is a section of $\mathcal{S} \times_{\delta} \mathcal{U}$ and that for some $c_{0} \in C(X)$ the following holds:

$$
\sigma_{c}\left(U_{c}\right) \subset K_{\delta(c)} \forall c \in C(X) \text { with } c \subsetneq c_{0}
$$

There is a continuous map $\phi_{c_{0}}:[0,1] \times U_{c_{0}} \rightarrow \mathcal{S}_{\delta\left(c_{0}\right)}$ satisfying:

(1) $\phi_{c_{0}}(0, x)=\sigma_{c_{0}}(x)$ for all $x \in U_{c_{0}}$,

(2) $\left(\phi_{c_{0}}(t, x), \sigma_{c}(x)\right) \in \mathcal{R}_{\delta\left(c_{0}\right), \delta(c)}$ for all $c_{0} \supsetneq c, x \in V_{c_{0}} \cap V_{c}$ and $t$,

(3) $\phi_{c_{0}}(t, x)=\sigma_{c_{0}}(x)$ for all $x$ outside some compact set $C \subset U_{c_{0}}$ and all $x \in U_{c_{0}}$ with $\sigma_{c_{0}}(x) \in K_{\delta\left(c_{0}\right)}$, and

(4) $\phi_{c_{0}}(1, x) \in K_{\delta\left(c_{0}\right)}$ for all $x \in V_{c_{0}}$. 
Before proving Lemma A.4 we establish some terminology. Suppose that $\phi: X \rightarrow Y$ is a map between two CW-complexes and that for some (countable) set of indices $i, j, \ldots$ we have homotopies

$$
\phi_{i}:[0,1] \times X \rightarrow Y
$$

with $\phi_{i}(0, x)=\phi(x)$ for all $x \in X$. We say that the homotopies $\phi_{i}$ do not interfere with each other if for each $i$ there is a compact set $C_{i} \subset X$ of satisfying the following two properties:

- $\quad C_{i} \cap C_{j}=\varnothing$ for all $i \neq j$ and no sequence $\left(x_{i}\right)$ with $x_{i} \in C_{i}$ has a convergent subsequence.

- $\phi_{i}(t, x)=\phi(x)$ for all $x \notin C_{i}$ and all $t$.

If the homotopies $\phi_{i}$ do not interfere with each other, then the map

$$
\Phi:[0,1] \times X \rightarrow Y
$$

given by $\Phi(t, x)=\phi_{i}(t, x)$ for $x \in K_{i}$ and $\Phi(t, x)=\phi(x)$ for $x \notin \bigcup_{i} C_{i}$ is continuous. We will refer to $\Phi$ as the combination of the homotopies $\phi_{i}$.

Continuing with the same notation suppose that we have maps

$$
\Phi, \Phi^{\prime}:[0,1] \times X \rightarrow Y
$$

with $\Phi(1, x)=\Phi^{\prime}(0, x)$ for all $x \in X$. The continuous map $\Phi^{\prime \prime}:[0,1] \times X \rightarrow Y$ given by $\Phi^{\prime \prime}(t, x)=\Phi(2 t, x)$ for $t \leq \frac{1}{2}$ and $\Phi^{\prime \prime}(t, x)=\Phi^{\prime}(2 t-1, x)$ for $t \geq \frac{1}{2}$ is the juxtaposition of $\Phi$ and $\Phi^{\prime}$.

Proof of Lemma A.4 Let $D=\operatorname{dim}\left(c_{0}\right)$ be the dimension of $c_{0}$ and choose for $i=0, \ldots, D$ a regular cover $\mathcal{U}^{i}=\left\{U_{c}^{i}\right\}_{c \in C(X)}$ with $\mathcal{U}^{0}=\mathcal{U}, \mathcal{U}^{D}=\mathcal{V}$ and such that $U_{c}^{i+1}$ is relatively compact in $U_{c}^{i}$ for all $c \in C(X)$.

Suppose that $c \subsetneq c_{0}$ is a codimension 1 face of $c_{0}$. Since the relative retraction property is satisfied, we can apply Lemma A.3 to $\delta\left(c_{0}\right) \geq \delta(c)$, the open set $U_{c_{0}}^{0} \cap U_{c}^{0}$ and the restrictions of $\sigma_{c_{0}}$ and $\sigma_{c}$ to $U_{c_{0}}^{0} \cap U_{c}^{0}$. Noticing that $U_{c_{0}}^{1} \cap U_{c}^{1}$ is relatively compact in $U_{c_{0}}^{0} \cap U_{c}^{0}$ we obtain hence a continuous map

$$
\phi_{c}:[0,1] \times\left(U_{c_{0}}^{0} \cap U_{c}^{0}\right) \rightarrow S_{\delta\left(c_{0}\right)}
$$

satisfying:

(1) $\phi_{c}(0, x)=\sigma_{c_{0}}(x)$ for all $x \in U_{c_{0}}^{0} \cap U_{c}^{0}$,

(2) $\left(\phi_{c}(t, x), \sigma_{c}(x)\right) \in \mathcal{R}_{\delta\left(c_{0}\right), \delta(c)}$ for all $x \in U_{c_{0}}^{0} \cap U_{c}^{0}$ and $t$, 
(3) $\phi_{c}(t, x)=\sigma_{c_{0}}(x)$ for all $x$ outside some compact set $C \subset U_{c_{0}}^{0} \cap U_{c}^{0}$ and all $x \in U_{c_{0}}^{0} \cap U_{c}^{0}$ with $\sigma_{0}(x) \in K_{\delta\left(c_{0}\right)}$, and

(4) $\phi_{c}(1, x) \in K_{\delta\left(c_{0}\right)}$ for all $x \in U_{c_{0}}^{1} \cap U_{c}^{1}$.

Notice that defining $\phi_{c}(t, x)=\sigma_{c_{0}}(x)$ for all $x \in U_{c_{0}}^{0} \backslash U_{c}^{0}$, we obtain a continuous map $\phi_{c}$ defined on the whole of $[0,1] \times U_{c_{0}}^{0}$. Suppose now that we are given a further proper face $\bar{c}$ of $c_{0}$ with $U_{c_{0}}^{0} \cap U_{c}^{0} \cap U_{\bar{c}}^{0} \neq \varnothing$ and notice that this implies that $c_{0} \supset c \supset \bar{c}$. We obtain from (2) the assumption that $\left\{\sigma_{c}\right\}_{c \in C(X)}$ is a section, and from the transitivity of $\mathcal{R}$ that

$$
\left(\phi_{c}(t, x), \sigma_{\bar{c}}(x)\right) \in \mathcal{R}_{\delta\left(c_{0}\right), \delta(\bar{c})}
$$

for all $x \in U_{c_{0}}^{0} \cap U_{c}^{0} \cap U_{\bar{c}}^{0}$. On the other hand, if $x \in\left(U_{c_{0}}^{0} \backslash U_{c}^{0}\right) \cap U_{\bar{c}}^{0}$ we have by definition that $\phi_{c}(t, x)=\sigma_{c_{0}}(x)$; it follows that (A.1) is actually satisfied for all $x \in U_{c_{0}}^{0} \cap U_{\bar{c}}^{0}$ and hence:

$$
\left(\phi_{c}(t, x), \sigma_{\bar{c}}(x)\right) \in \mathcal{R}_{\delta\left(c_{0}\right), \delta(\bar{c})} \text { for all } c_{0} \supsetneq \bar{c}, x \in U_{c_{0}}^{0} \cap U_{\bar{c}}^{0} \text { and } t .
$$

Suppose that we are given a second codimension one face $c^{\prime}$ of $c_{0}$. Since $U_{c}^{0} \cap U_{c^{\prime}}^{0}=\varnothing$ the homotopies $\phi_{c}$ and $\phi_{c^{\prime}}$ do not interfere with each other. Combining all the homotopies corresponding to all the codimension one faces of $c_{0}$, we have then that for $d=1$ there is a map

$$
\psi^{d}:[0,1] \times U_{c_{0}} \rightarrow S_{\delta\left(c_{0}\right)}
$$

satisfying:

(a) $\quad \psi^{d}(0, x)=\sigma_{c_{0}}(x)$ for all $x \in U_{c_{0}}=U_{c_{0}}^{0}$,

(b) $\quad\left(\psi^{d}(t, x), \sigma_{c}(x)\right) \in \mathcal{R}_{\delta\left(c_{0}\right), \delta(c)}$ for all $c_{0} \supsetneq c, x \in U_{c_{0}}^{d-1} \cap U_{c}^{d-1}$ and $t$,

(c) $\psi^{d}(t, x)=\sigma_{c_{0}}(x)$ for all $x$ outside some compact set $C \subset U_{c_{0}}$ and for all $x \in U_{c_{0}}$ with $\sigma_{c_{0}}(x) \in K_{\delta\left(c_{0}\right)}$, and

(d) $\quad \psi^{d}(1, x) \in K_{\delta(c)}$ for all $x \in U_{c_{0}}^{d} \cap U_{c}^{d}$ where $c \subsetneq c_{0}$ has at most codimension $d$.

Arguing by induction, we may assume that for some $d \geq 1$ we have a map $\psi^{d}:[0,1] \times$ $U_{c_{0}} \rightarrow S_{\delta\left(c_{0}\right)}$ satisfying (a), (b), (c) and (d). Suppose that $c \subset c_{0}$ is a codimension $d+1$ face. Applying Lemma A.3 to $\delta\left(c_{0}\right) \geq \delta(c)$, the open set $U_{c_{0}}^{d} \cap U_{c}^{d}$ and the restrictions of $\psi^{d}(1, \cdot)$ and $\sigma_{c}$ to $U_{c_{0}}^{d} \cap U_{c}^{d}$, we obtain again a map

$$
\phi_{c}:[0,1] \times\left(U_{c_{0}}^{d} \cap U_{c}^{d}\right) \rightarrow S_{\delta\left(c_{0}\right)}
$$

satisfying:

(1) $\phi_{c}(0, x)=\psi^{d}(1, x)$ for all $x \in U_{c_{0}}^{d} \cap U_{c}^{d}$, 
(2) $\left(\phi_{c}(t, x), \sigma_{c}(x)\right) \in \mathcal{R}_{\delta\left(c_{0}\right), \delta(c)}$ for all $x \in U_{c_{0}}^{d} \cap U_{c}^{d}$ and $t$,

(3) $\phi_{c}(t, x)=\psi^{d}(1, x)$ for all $x$ outside some compact set $C \subset U_{c_{0}}^{d} \cap U_{c}^{d}$ and all $x \in U_{c_{0}}^{d} \cap U_{c}^{d}$ with $\psi^{d}(1, x) \in K_{\delta\left(c_{0}\right)}$, and

(4) $\phi_{c}(1, x) \in K_{\delta\left(c_{0}\right)}$ for all $x \in U_{c_{0}}^{d+1} \cap U_{c}^{d+1}$.

As above, we set $\phi_{c}(t, x)=\psi_{d}(1, x)$ for all $x \in U_{c_{0}} \backslash U_{c}^{d}$ obtaining thus a continuous extension:

$$
\phi_{c}:[0,1] \times U_{c_{0}} \rightarrow S_{\delta\left(c_{0}\right)}
$$

We claim that $\phi_{c}$ satisfies the following strengthening of $(\hat{2})$ :

$\left(\hat{2}^{\prime}\right) \quad\left(\phi_{c}(t, x), \sigma_{\bar{c}}(x)\right) \in \mathcal{R}_{\delta\left(c_{0}\right), \delta(\bar{c})}$ for all $c_{0} \supsetneq \bar{c}, x \in U_{c_{0}}^{d} \cap U_{\bar{c}}^{d}$ and $t$.

If $\bar{c} \subset c$ then, as above, the transitivity of the set $\mathcal{R}$ implies that $\left(\hat{2}^{\prime}\right)$ holds for all $x \in U_{c_{0}}^{d} \cap U_{\bar{c}}^{d}$ and $t$. Suppose now that $c \subsetneq \bar{c}$. By (d) we have that $\psi^{d}(1, x) \in K_{\delta\left(c_{0}\right)}$ for all $x \in U_{c_{0}}^{d} \cap U_{\bar{c}}^{d}$. It follows hence from ( $(\widehat{3})$ and (b) that $\left(\phi_{c}(t, x), \sigma_{\bar{c}}(x)\right) \in \mathcal{R}_{\delta\left(c_{0}\right), \delta(\bar{c})}$ for all $x \in U_{c_{0}}^{d} \cap U_{\bar{c}}^{d}$ and $t$. Finally observe that $U_{c}^{d} \cap U_{\bar{c}}^{d}=\varnothing$ if $\bar{c}$ is neither contained in nor contains $c$. It follows that for any such $c$ we have $\phi_{c}(t, x)=\psi^{d}(1, x)$ for all $x \in U_{c_{0}} \cap U_{c}$ and all $t$. The validity of $\left(\hat{2}^{\prime}\right)$ for $x \in U_{c_{0}}^{d} \cap U_{\bar{c}}^{d}$ follows hence from (b). We have proved that $\phi^{c}$ satisfies $\left(\hat{2}^{\prime}\right)$.

As above, the maps $\phi_{c}$ and $\phi_{c^{\prime}}$ do not interfere with each other for any two distinct co-dimension $d+1$ faces $c$ and $c^{\prime}$ of $c_{0}$. The juxtaposition

$$
\psi^{d+1}:[0,1] \times U_{c_{0}} \rightarrow S_{\delta(c)}
$$

of $\psi^{d}$ and the combination of all the homotopies $\phi_{c}$ with $c$ a co-dimension $d+1$ face of $c_{0}$ satisfies (a), (b), (c) and (d) for $d+1$.

Recall that $\mathcal{U}^{D}=\mathcal{V}$; repeating this process $D$ times we obtain hence a map

$$
\psi=\psi^{D}:[0,1] \times U_{c_{0}} \rightarrow S_{\delta\left(c_{0}\right)}
$$

satisfying:

$\left(\mathrm{a}^{\prime}\right) \quad \psi(0, x)=\sigma_{c_{0}}(x)$ for all $x \in U_{c_{0}}$,

$\left(\mathrm{b}^{\prime}\right) \quad\left(\psi(t, x), \sigma_{c}(x)\right) \in \mathcal{R}_{\delta\left(c_{0}\right), \delta(c)}$ for all $c_{0} \supsetneq c, x \in V_{c_{0}} \cap V_{c}$ and $t$,

$\left(\mathrm{c}^{\prime}\right) \quad \psi(t, x)=\sigma_{c_{0}}(x)$ for all $x$ outside some compact set $C \subset U_{c_{0}}$ and for all $x \in U_{c_{0}}$ with $\sigma_{c_{0}}(x) \in K_{\delta\left(c_{0}\right)}$, and

$\left(\mathrm{d}^{\prime}\right) \quad \psi(1, x) \in K_{\delta\left(c_{0}\right)}$ for all $x \in V_{c_{0}} \cap\left(\bigcup_{c \subsetneq c_{0}} V_{c}\right)$. 
So far, we have only used the second part of the relative retraction property (RR2). Taking now into account $\left(\mathrm{d}^{\prime}\right)$ and that the inclusion of $K_{\delta\left(c_{0}\right)} \rightarrow S_{\delta\left(c_{0}\right)}$ is a homotopy equivalence, we can find a homotopy

$$
\psi^{\prime}:[0,1] \times U_{c_{0}} \rightarrow S_{\delta\left(c_{0}\right)}
$$

relative to $V_{c_{0}} \cap\left(\bigcup_{c \varsubsetneqq c_{0}} V_{c}\right)$, to the complement of a compact subset of $U_{c_{0}}$, starting with $\psi^{\prime}(0, x)=\psi(1, x)$, such that $\psi^{\prime}(1, x) \in K_{\delta\left(c_{0}\right)}$ for all $x \in V_{c_{0}}$. Juxtaposing $\psi$ and $\psi^{\prime}$ we obtain finally a continuous map $\phi_{c_{0}}:[0,1] \times U_{c_{0}} \rightarrow S_{\delta\left(c_{0}\right)}$ satisfying conditions (1), (2), (3) and (4) in the statement of Lemma A.4.

\section{A.4 Proof of Proposition A.1}

Starting with the proof of Proposition A.1, let $D=\operatorname{dim} X$ and choose a regular cover $\mathcal{U}^{i}=\left\{U_{c}^{i}\right\}_{c \in C(X)}$, for $i=0, \ldots, D$ with $\mathcal{U}^{0}=\mathcal{U}, \mathcal{U}^{D}=\mathcal{V}$, such that $U_{c}^{i+1}$ is relatively compact in $U_{c}^{i}$ for all $c \in C(X)$. We will denote by $X^{(d)}$ the $d$-skeleton of $X$, ie, the simplicial complex consisting of all the cells of dimension at most $d$; the set of cells of dimension precisely $d$ is denoted by $C_{d}(X)$.

Again we will argue by induction. Observing that $X^{(-1)}=\varnothing$, we may suppose that for some $d \geq 0$ we have $\sigma_{c}\left(U_{c}^{d-1}\right) \subset K_{\delta(c)}$ for all $c \in C(X)$ of dimension at most $d-1$.

We will show that there is a homotopy of sections, constant on $W$, which starts with the restriction of $\left\{\sigma_{c}\right\}_{c \in C(X)}$ to $\mathcal{U}^{d}$ and ends with a section $\left\{\sigma_{c}^{\prime}\right\}_{c \in C(X)}$ of $\mathcal{S} \times_{\delta} \mathcal{U}^{d}$ with $\sigma_{c}^{\prime}\left(U_{c}^{d}\right) \subset K_{\delta(c)}$ for all $c \in C\left(X^{(d)}\right)$.

Let $c_{0} \in C_{d}(X)$ be a $d$-dimensional cell. Consider the cells $c_{1}, \ldots, c_{r} \in C(X)$ that are properly contained in $c_{0}$ and notice that $\delta\left(c_{0}\right) \geq \delta\left(c_{i}\right)$ for all $i$. The retraction lemma, Lemma A.4, yields a map

$$
\bar{\sigma}_{c_{0}}:[0,1] \times U_{c_{0}}^{d-1} \rightarrow \mathcal{S}_{\delta\left(c_{0}\right)}, \quad(t, x) \mapsto \bar{\sigma}_{c_{0}}^{t}(x)
$$

with $\bar{\sigma}_{c_{0}}^{0}=\sigma_{c_{0}}, \bar{\sigma}_{c_{0}}^{1}\left(U_{c_{0}}^{d}\right) \subset K_{\delta\left(c_{0}\right)}$, such that:

- $\left(\bar{\sigma}_{c_{0}}^{t}(x), \sigma_{c_{i}}(x)\right) \in \mathcal{R}_{\delta\left(c_{0}\right), \delta\left(c_{i}\right)}$ for all $i>0, x \in U_{c_{0}}^{d} \cap U_{c_{i}}^{d}$, and $t$.

- $\bar{\sigma}_{c_{0}}^{t}(t, x)=\sigma_{c_{0}}(x)$ for all $x$ outside some compact set $C \subset U_{c_{0}}^{d-1}$ and all $x \in W \cap U_{c_{0}}^{d-1}$.

The Lifting Lemma A.2 implies that there is a section $\left\{\sigma_{c}^{\prime}\right\}_{c \in C(X)}$ of $\mathcal{S} \times_{\delta} \mathcal{U}^{d}$ with $\sigma_{c_{0}}^{\prime}=\bar{\sigma}_{c_{0}}^{1}$ and a homotopy $\left\{\sigma_{c}^{t}\right\}_{c \in C(X)}$ supported by $K$ between $\left\{\sigma_{c}\right\}_{c \in C(X)}$ and $\left\{\sigma_{c}^{\prime}\right\}_{c \in C(X)}$. Moreover, the homotopy $t \mapsto \sigma_{c}^{t}$ is constant unless $c_{0} \subset c$. In particular, $\sigma_{c}^{\prime}=\sigma_{c}$ for all $c \in C\left(X^{(d-1)}\right)$. 
Since $U_{c}^{d} \cap U_{c^{\prime}}^{d}=\varnothing$ for every other $c_{0}^{\prime} \in C_{d}(X)$, we deduce that the homotopy just constructed for $c_{0}$ and the corresponding one for $c_{0}^{\prime}$ do not interfere with each other. Combining the homotopies corresponding to all the $d$-dimensional cells, we obtain that the restriction to $\mathcal{U}^{d}$ of the section $\left\{\sigma_{c}\right\}$ is homotopic, through a homotopy constant on $W$, to a section $\left\{\sigma_{c}^{\prime}\right\}$ of $\mathcal{S} \times{ }_{\delta} \mathcal{U}^{d}$, with $\sigma_{c}\left(U_{c}^{d}\right) \subset K_{\delta(c)}$ for all $c \in C(X)$ with $\operatorname{dim}(c) \leq d$. This concludes the proof of the induction step and hence the proof of Proposition A.1.

\section{A.5 Proof of Proposition 5.3}

Let $G$ be the group of complex or real points of a reductive algebraic group (defined over $\mathbb{R}$ in the latter case), $K \subset G$ a maximal compact subgroup, and let $G_{0} \subset G$ and $K_{0} \subset G$ the respective identity components. Let also $\mathcal{G}$ and $\mathcal{K}$ be the manifolds of all well-centered subgroups of $G$ and $K$. Consider $\pi_{0}(\mathcal{G})$ with the partial ordering introduced in Section 3.5, and $\mathcal{R}_{G}$ and $\mathcal{O}_{G}$ as defined in (5.1) and (5.2). By Lemma 5.2, $\left(\mathcal{R}_{G}, \mathcal{O}_{G}\right)$ is the set of fibered relation pairs. It follows directly from the definitions that $\mathcal{R}_{G}$ is transitive and reflexive:

Lemma A.5 The set $\mathcal{R}_{G}$ of fibered relations is transitive and reflexive.

We prove that $\mathcal{R}_{G}$ and $\left(\mathcal{R}_{G}, \mathcal{O}_{G}\right)$ satisfy, respectively, the simultaneous lifting and relative retraction properties.

Lemma A.6 The set $\mathcal{R}_{G}$ satisfies the simultaneous lifting property.

Proof Suppose that $\mathcal{G}_{0}, \mathcal{G}_{1}, \ldots, \mathcal{G}_{r} \in \pi_{0}(\mathcal{G})$ are such that $\mathcal{G}_{i} \geq \mathcal{G}_{0}$ for all $i$ and that $W_{0}, \ldots, W_{r}$ are contractible open sets in a CW-complex with $W_{i} \subset W_{0}$ for all $i$. Finally suppose that we have for each $i=0, \ldots, r$ a continuous map $\sigma_{i}$ : $W_{i} \rightarrow \mathcal{G}_{i}$ with $\left(\sigma_{i}(x), \sigma_{j}(x)\right) \in \mathcal{R}_{\mathcal{G}_{i}, \mathcal{G}_{j}}$ for all $i, j \in\{0, \ldots, r\}$ with $\mathcal{G}_{i} \geq \mathcal{G}_{j}$ and all $x \in W_{i} \cap W_{j}$.

Let also $\phi_{0}:[0,1] \times W_{0} \rightarrow \mathcal{G}_{0}$ be continuous with $\phi_{0}(0, x)=\sigma_{0}(x)$. Choosing a base point $A \in \mathcal{G}_{0}$ we consider the map

$$
p: G_{0} \rightarrow \mathcal{G}_{0}, \quad g \mapsto g A g^{-1} .
$$

Notice that $p: G_{0} \rightarrow \mathcal{G}_{0}$ is a fiber bundle with fiber $\mathcal{N}_{G_{0}}(A)=\mathcal{H}_{G}(A) \cap G_{0}$; hence $p$ is a fibration. It follows from the contractibility of $W_{0}$ that there is map

$$
\alpha:[0,1] \times W_{0} \rightarrow G_{0}
$$


such that the following diagram commutes:

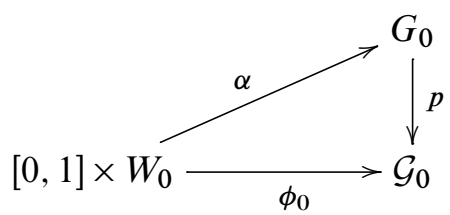

Moreover, if there is a compact set $K \subset W_{0}$ with $\phi_{0}(t, x)=\phi_{0}(0, x)$ for all $x \notin K$ and all $t$ then we may assume also that $\alpha(t, x)=\alpha(0, x)$ for all $x \notin K$ and all $t$.

We consider now the map

$$
\beta:[0,1] \times W_{0} \rightarrow G_{0}, \quad \beta(t, x)=\alpha(t, x) \alpha(0, x)^{-1} .
$$

Observe that $\beta(t, x)=$ Id for all $x \notin K$ and all $t$, that $\beta(0, x)=$ Id for all $x \in W_{0}$ and that

$$
\begin{aligned}
\phi(t, x)=\alpha(t, x) A \alpha(t, x)^{-1} & =\alpha(t, x) \alpha(0, x)^{-1} \sigma_{0}(x) \alpha(0, x) \alpha(t, x)^{-1} \\
& =\beta(t, x) \sigma_{0}(x) \beta(t, x)^{-1}
\end{aligned}
$$

for all $x \in W_{0}$ and all $t$.

For $i=1, \ldots, r$ we consider the map

$$
\phi_{i}:[0,1] \times W_{i} \rightarrow \mathcal{G}_{i}, \quad \phi_{i}(t, x)=\beta(t, x) \sigma_{i}(x) \beta(t, x)^{-1}
$$

and notice that $t \mapsto \phi_{i}(t, x)$ is constant for $x$ outside of $K \cap W_{i}$. Also, it follows directly from (5.1), the definition of $\mathcal{R}_{\mathcal{G}_{i}, \mathcal{G}_{j}}$, that

$$
\left(\phi_{i}(t, x), \phi_{j}(t, x)\right) \in \mathcal{R}_{\mathcal{G}_{i}, \mathcal{G}_{j}}
$$

for all $t$, all $i, j \in\{0, \ldots, r\}$ with $\mathcal{G}_{i} \geq \mathcal{G}_{j}$ and all $x \in W_{i} \cap W_{j}$. We have proved that $\mathcal{R}_{G}$ has the simultaneous lifting property.

Lemma A.7 The set $\left(\mathcal{R}_{G}, \mathcal{O}_{G}\right)$ satisfies the relative retraction property.

Proof To begin with recall that for every $\mathcal{G}_{0} \in \pi_{0}(\mathcal{G})$ the inclusion $\mathcal{K}_{0} \rightarrow \mathcal{G}_{0}$ is a homotopy equivalence by Proposition 3.11; in other words, the first half (RR1) of the relative retraction property is satisfied. Fixing a base point $\left(A_{0}, A_{1}\right) \in \mathcal{R}_{\mathcal{G}_{0}, \mathcal{G}_{1}} \cap$ $\left(\mathcal{K}_{0} \times \mathcal{K}_{1}\right)$ we obtain from Lemma 3.13 and Lemma 3.14 that the fibrations

$$
\mathcal{R} \cap\left(\mathcal{G}_{0} \times \mathcal{K}_{1}\right) \rightarrow \mathcal{K}_{1} \quad \text { and } \quad \mathcal{R} \cap\left(\mathcal{K}_{0} \times \mathcal{K}_{1}\right) \rightarrow \mathcal{K}_{1}
$$

are locally trivial fiber bundles with fibers

$$
\operatorname{Trans}_{G_{0}}\left(A_{1}, A_{0}\right) / \mathcal{N}_{G_{0}}\left(A_{1}\right) \quad \text { and } \quad \operatorname{Trans}_{K_{0}}\left(A_{1}, A_{0}\right) / \mathcal{N}_{K_{0}}\left(A_{1}\right) \text {. }
$$


In order to prove that (RR2) is satisfied, it suffices to show that the inclusion

$$
\operatorname{Trans}_{K_{0}}\left(A_{1}, A_{0}\right) / \mathcal{N}_{K_{0}}\left(A_{1}\right) \rightarrow \operatorname{Trans}_{G_{0}}\left(A_{1}, A_{0}\right) / \mathcal{N}_{G_{0}}\left(A_{1}\right)
$$

is a homotopy equivalence. Consider the commuting diagram:

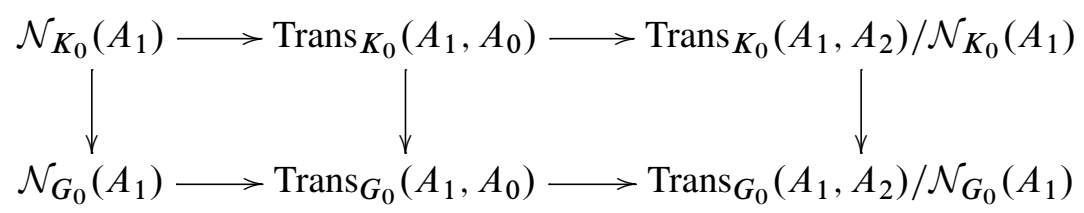

The rows are fibrations and the first two columns are homotopy equivalences by Lemma 3.4. It follows that the third column is also a homotopy equivalence, as we needed to show. This concludes the proof of Lemma A.7.

Proposition 5.3 follows directly from Lemmas A.5, A.6 and A.7 and Proposition A.1.

The second author wishes to quote Günter Netzer, who, to the best of his understanding, was paraphrasing Berti Vogts: Deutsche Tugenden haben wir immer noch am besten. Gemeint waren Kampf, Kondition, nimmermüde Einsatzbereitschaft, Durchsetzungsvermögen und Siegeswille.

\section{References}

[1] A Adem, F R Cohen, Commuting elements and spaces of homomorphisms, Math. Ann. 338 (2007) 587-626 MR2317932

[2] A Adem, F R Cohen, J M Gómez, Stable splittings, spaces of representations and almost commuting elements in Lie groups, Math. Proc. Cambridge Philos. Soc. 149 (2010) 455-490 MR2726729

[3] A Adem, F R Cohen, J M Gómez, Commuting elements in central products of special unitary groups, Proc. Edinb. Math. Soc. 56 (2013) 1-12 MR3021401

[4] A Adem, F R Cohen, E Torres-Giese, Commuting elements, simplicial spaces and filtrations of classifying spaces, Math. Proc. Cambridge Philos. Soc. 152 (2012) 91-114 MR2860418

[5] T J Baird, Cohomology of the space of commuting n-tuples in a compact Lie group, Algebr. Geom. Topol. 7 (2007) 737-754 MR2308962

[6] T J Baird, L C Jeffrey, P Selik, The space of commuting $n$-tuples in SU(2), to appear in Illinois J. Math.

[7] A Borel, Linear algebraic groups, 2nd edition, Graduate Texts in Mathematics 126, Springer, New York (1991) MR1102012 
[8] A Borel, Lie groups and linear algebraic groups, I: Complex and real groups, from: "Lie groups and automorphic forms", (L Ji, J-S Li, H W Xu, S-T Yau, editors), AMS/IP Stud. Adv. Math. 37, Amer. Math. Soc. (2006) 1-49 MR2272918

[9] A Borel, R Friedman, J W Morgan, Almost commuting elements in compact Lie groups, Mem. Amer. Math. Soc. 157 (2002) 136 MR1895253

[10] M Brion, Introduction to actions of algebraic groups Available at http:// ww-fourier.ujf-grenoble.fr/ mbrion

[11] R Charney, An introduction to right-angled Artin groups, Geom. Dedicata 125 (2007) 141-158 MR2322545

[12] M C Crabb, Spaces of commuting elements in SU(2), Proc. Edinb. Math. Soc. 54 (2011) 67-75 MR2764406

[13] M Gerstenhaber, On dominance and varieties of commuting matrices, Ann. of Math. 73 (1961) 324-348 MR0132079

[14] W M Goldman, Topological components of spaces of representations, Invent. Math. 93 (1988) 557-607 MR952283

[15] J M Gómez, A Pettet, J Souto, On the fundamental group of $\operatorname{Hom}\left(\mathbb{Z}^{k}, G\right)$, to appear in Math. Zeitschrift.

[16] V V Gorbatsevich, A L Onishchik, Lie transformation groups, from: "Lie groups and Lie algebras, I", Encyclopaedia Math. Sci. 20, Springer, Berlin (1993) 95-235

[17] R M Guralnick, A note on commuting pairs of matrices, Linear and Multilinear Algebra 31 (1992) 71-75 MR1199042

[18] A Hatcher, Algebraic topology, Cambridge Univ. Press (2002) MR1867354

[19] G Higuera, On the space of commuting orthogonal matrices (2013) preprint Available at http://www.math.ubc.ca/ galo/commutingtuples.pdf

[20] K R Hofmann, Triangulation of locally semi-algebraic spaces, $\mathrm{PhD}$ thesis, University of Michigan (2009)

[21] L C Jeffrey, Flat connections on oriented 2-manifolds, Bull. London Math. Soc. 37 (2005) 1-14 MR2105813

[22] V G Kac, A V Smilga, Vacuum structure in supersymmetric Yang-Mills theories with any gauge group, from: "The many faces of the superworld", (M Shifman, editor), World Sci. Publ. (2000) 185-234 MR1885976

[23] D Luna, Slices étales, from: "Sur les groupes algébriques”, Bull. Soc. Math. France, Paris 33, Soc. Math. France, Paris (1973) 81-105 MR0342523

[24] G D Mostow, On a conjecture of Montgomery, Ann. of Math. 65 (1957) 513-516 MR0087039

[25] D Mumford, The red book of varieties and schemes, Lecture Notes in Mathematics 1358, Springer, Berlin (1999) MR1748380 
[26] A Parshin, I R Shafarevich, Algebraic geometry, IV: Linear algebraic groups, invariant theory, Encyclopaedia Math. Sci. 55, Springer, Berlin (1994) MR1309681

[27] R W Richardson, Principal orbit types for algebraic transformation spaces in characteristic zero, Invent. Math. 16 (1972) 6-14 MR0294336

[28] R W Richardson, Affine coset spaces of reductive algebraic groups, Bull. London Math. Soc. 9 (1977) 38-41 MR0437549

[29] R W Richardson, Commuting varieties of semisimple Lie algebras and algebraic groups, Compositio Math. 38 (1979) 311-327 MR535074

[30] R W Richardson, Conjugacy classes of $n$-tuples in Lie algebras and algebraic groups, Duke Math. J. 57 (1988) 1-35 MR952224

[31] J-P Serre, Complex semisimple Lie algebras, Springer, Berlin (2001) MR1808366

[32] J-P Serre, Galois cohomology, Springer, Berlin (2002) MR1867431

[33] I R Shafarevich, Basic algebraic geometry, 1: Varieties in projective space, 2nd edition, Springer, Berlin (1994) MR1328833

[34] J Souto, A remark on the homotopy equivalence between $\mathrm{SU}_{n}$ and $\mathrm{SL}_{n} \mathbb{C}$ arXiv: 1008.0816

[35] A Tarski, A decision method for elementary algebra and geometry, 2nd edition, University of California Press, Berkeley and Los Angeles, CA (1951) MR0044472

[36] R Thom, Ensembles et morphismes stratifiés, Bull. Amer. Math. Soc. 75 (1969) 240284 MR0239613

[37] E Torres-Giese, D Sjerve, Fundamental groups of commuting elements in Lie groups, Bull. Lond. Math. Soc. 40 (2008) 65-76 MR2409179

[38] H Whitney, Elementary structure of real algebraic varieties, Ann. of Math. 66 (1957) 545-556 MR0095844

[39] H Whitney, Tangents to an analytic variety, Ann. of Math. 81 (1965) 496-549 MR0192520

[40] E Witten, Constraints on supersymmetry breaking, Nuclear Phys. B 202 (1982) 253316 MR668987

[41] E Witten, Toroidal compactification without vector structure, J. High Energy Phys. (1998) 43 MR1615617

Department of Mathematics, University of British Columbia

1984 Mathematics Road, Vancouver, BC V6T 1Z2, Canada

alexandra@math.ubc.ca, jsouto@math.ubc.ca

http://www.math.ubc.ca/ jsouto

Proposed: Benson Farb

Received: 7 June 2012

Seconded: Jesper Grodal, Leonid Polterovich

Accepted: 5 May 2013 